\title{
Gauge theory deformations and novel Yang-Mills Chern-Simons field theories with torsion
}

\author{
Stephen C. Ancd* \\ Department of Mathematics, Brock University, \\ St Catharines, Ontario, L2S 3A1, Canada
}

(Dated: August 6, 2004) 


\begin{abstract}
A basic problem of classical field theory, which has attracted growing attention over the past decade, is to find and classify all nonlinear deformations of linear abelian gauge theories. The physical interest in studying deformations is to address uniqueness of known nonlinear interactions of gauge fields and to look systematically for theoretical possibilities for new interactions. Mathematically, the study of deformations aims to understand the rigidity of the nonlinear structure of gauge field theories and to uncover new types of nonlinear geometrical structures.

The first part of this paper summarizes and significantly elaborates a field-theoretic deformation method developed in earlier work. Some key contributions presented here are, firstly, that the determining equations for deformation terms are shown to have an elegant formulation using Lie derivatives in the jet space associated with the gauge field variables. Secondly, the obstructions (integrability conditions) that must be satisfied by lowest-order deformations terms for existence of a deformation to higher orders are explicitly identified. Most importantly, a universal geometrical structure common to a large class of nonlinear gauge theory examples is uncovered. This structure is derived geometrically from the deformed gauge symmetry and is characterized by a covariant derivative operator plus a nonlinear field strength, related through the curvature of the covariant derivative. The scope of these results encompasses Yang-Mills theory, Freedman-Townsend theory, Einstein gravity theory, in addition to their many interesting types of novel generalizations that have been found in the past several years.

The second part of the paper presents a new geometrical type of Yang-Mills generalization in three dimensions motivated from considering torsion in the context of nonlinear sigma models with Lie group targets (chiral theories). The generalization is derived by a deformation analysis of linear abelian Yang-Mills Chern-Simons gauge theory. Torsion is introduced geometrically through a duality with chiral models obtained from the chiral field form of self-dual $2+2$ dimensional YangMills theory under reduction to $2+1$ dimensions. Field-theoretic and geometric features of the resulting nonlinear gauge theories with torsion are discussed.
\end{abstract}

Keywords: gauge theory, nonlinear deformation, Yang-Mills field theory, Freedman-Townsend field theory, Chern-Simons field theory, torsion, nonlinear sigma model

*Electronic address: sanco@brocku.ca 


\section{INTRODUCTION AND SUMMARY}

Classical gauge field theories are widely studied in many areas of mathematics, and their importance in physics almost needs no comment. They provide the starting point for formulations of physically important theories of quantum fields; in particular, for the case of electromagnetic and gravitational fields, they describe the dynamics of macroscopic quantum states in the classical regime where quantum effects are not significant.

Looked at from the view point of geometry, classical gauge theories have a rich nonlinear structure - field variables typically are sections of a vector bundle or group bundle over spacetime, equations of motion for the fields usually involve a connection, covariant derivative, and curvature tensor on this bundle, while gauge symmetries of the fields are described by an infinite-dimensional Lie group action or local bundle automorphisms. One way to characterize all this structure is by the idea of deformations of linear abelian gauge theories.

A prototype example is non-abelian Yang-Mills theory. The Yang-Mills field is mathematically defined as a connection 1-form in a semisimple Lie group bundle on spacetime, in which the Yang-Mills gauge symmetry is a local automorphism given by the group action of the bundle. If a linearization around a flat connection is considered, the theory reduces to a set of linear electromagnetic field equations and corresponding spin-one gauge symmetries. Non-abelian Yang-Mills theory describes a nonlinear deformation of the structure of this linear abelian (electromagnetic field) theory [1, 2, 3]. Another prototypical example is Einstein gravity theory. The gravitational field is a metric tensor on spacetime to which is naturally associated a metric-connection in the tangent bundle, and the gauge symmetries arise from diffeomorphisms acting on the spacetime manifold. Linearization around a flat metric reduces the theory to the linear gravity wave equation and its accompanying abelian spin-two gauge symmetry. Einstein gravity theory represents a highly nonlinear deformation of the structure of this linear abelian (graviton field) theory [2, 3, 4, 5]. In both these examples, the gauge covariant field strength in the theory is simply the curvature defined geometrically from the field.

An interesting problem of classical field theory is to find and classify all nonlinear deformations of a given linear abelian gauge theory [6, 7, 8]. Deformations, in general, refer here to adding linear and higher power local terms to the abelian gauge symmetry, while also adding quadratic and higher power local terms to the linear field equation, such that 
there exists a gauge invariant local action functional for the deformed theory. Such a deformation is essentially nonlinear if the deformed Lagrangian is not equivalent, to within a local divergence, to the Lagrangian of the linear theory under any invertible local nonlinear field redefinitions. Deformations in which the abelian gauge symmetry remains undeformed (for example, by adding gauge invariant Pauli-type interaction terms to the Lagrangian) are regarded as trivial since the abelian gauge invariance (and, hence, geometrical structure) is unchanged. Importantly, the condition of gauge invariance can be used to formulate determining equations to solve for all allowed deformation terms order-by-order in powers of the fields, without any need for special assumptions or ansatzes on possible forms of the deformed field equations, gauge symmetries, and gauge group structure. There are two main formulations of the deformation determining equations - a direct, field-theoretic approach 6] where gauge invariance is expressed elegantly by Lie derivative equations for gauge symmetries, using a jet space formalism in terms of the gauge field variables; and a powerful BRST approach [7] based on the field-antifield formalism [8, 9, 10] in which the condition of a gauge invariant Lagrangian is encoded by the cohomology of a BRST operator.

The physical interest in studying deformations is to address uniqueness of known nonlinear interactions of gauge fields and to look systematically for theoretical possibilities for new interactions. Mathematically, the study of deformations aims to understand the rigidity of the nonlinear structure of gauge field theories and to uncover new types of nonlinear geometrical structures. Sparking this subject is the rich interplay between, on the one hand, differential geometry and deformations of graviton theory, connections on vector bundles and deformations of electromagnetic theory, and on the other hand, their obvious significance for many developments in mathematics and physics.

Indeed, the last decade has seen a large body of work on the study of nonlinear gauge theory deformations, which has yielded many interesting new kinds of generalizations of Yang-Mills theory and Einstein gravity theory both as classical theories of spin-one and spin-two fields and as geometrical theories of Lie-algebra valued 1-forms and algebra valued vielbeins or metrics. Rigidity results for these generalizations have been obtained as well 11, 12, 13, 14, 15, 16 ]

The first gravity generalizations to be found were motivated by aspects of classical supergravity theory [17] and describe nonlinear multi-graviton theories in four dimensions involving the introduction of various algebras in which the fields take values [14, 18]. Super- 
symmetric extensions of these generalizations have also been obtained, along with more novel extensions that involve non-commuting classical graviton fields (and non-anticommuting gravitino fields) [15]. Recently, an exotic type of multi-graviton theory with a parity-violating interaction that exists for commuting graviton fields only in three and five dimensions has been investigated [19, 20]. This exotic gravity generalization is most remarkable in that its gauge invariance is completely different than the familiar diffeomorphism invariance of Einstein gravity, in contrast to the previous generalizations which all feature diffeomorphism invariance extended to an algebra-valued setting.

Yang-Mills generalizations were first obtained for 1-form potentials in three dimensions 21, 22] and come from a Yang-Mills interaction combined with a Freedman-Townsend type interaction available for 1-forms only when the number of dimensions equals three. Recall, Freedman-Townsend theory [23] exists for $d-2$-form potentials in $d \geq 3$ dimensions, with the dual nonlinear field strength of the potential serving as a connection 1-form whose curvature vanishes due to the field equations; as is well known, because the connection is flat, Freedman-Townsend theory geometrically describes a dual form of principal chiral models. Extensions of this type of generalization of Yang-Mills theory to interacting 1-form and 2-form potentials in four dimensions [24], including a Chern-Simons type mass term, were later obtained [16]. (It should also be noted that gauge theories containing 2-form potentials are of active interest in supergravity contexts 25].) Subsequently a different type of generalization in four dimensions was derived in recent work [26] involving a ChaplineManton type interaction between 1-form and 2-form potentials combined with a Yang-Mills interaction and an extended Freedman-Townsend interaction. The gauge invariance in all these generalizations unifies the form of the familiar Yang-Mills and Freedman-Townsend gauge symmetries, leading to a nonlinear structure that mixes geometrical features of pure Yang-Mills and pure Freedman-Townsend theories in an interesting way. In particular, the combined Yang-Mills Chapline-Manton generalization exhibits a dual formulation that describes a principal chiral field with an exotic dilaton coupling to the Yang-Mills fields through a generalized Chern-class term [26].

In another direction, a novel Yang-Mills generalization involving a gravity-like interaction of 1-form potentials has been recently derived [27], in which the interaction is constructed using conserved currents associated with Killing vectors of spacetime. Its gauge invariance unites the familiar Yang-Mills type with the vielbein type found in Einstein gravity, giving 
a gauge theory of spacetime symmetries (Killing vectors) in a direct sense. However, the geometrical structure and gravity-like aspects of the theory remain to be explored more fully.

A main purpose of this paper will be to present a new geometrical type of Yang-Mills generalization in three dimensions motivated from considering torsion in the context of nonlinear sigma models [28]. Recall a nonlinear sigma field (also known as a wave map) is a function on spacetime taking values in a Riemannian target space e.g. an $n$-sphere or a compact simple Lie group (note the latter defines a chiral field model). The nonlinear structure of a sigma model is given jointly in terms of the spacetime metric and the symmetric (Riemannian) metric-connection on the target. More specifically, the field equation is a semilinear geometrical wave equation (sometimes called a wave map equation) consisting of the spacetime wave operator on the sigma field plus a quadratic interaction given by a product of sigma field gradients contracted with the target metric-connection and the spacetime metric tensor. This interaction readily generalizes to include torsion in the antisymmetric part of the connection on the target, if at the same time a skew tensor is added to the spacetime metric.

Torsion first arose in this context for $1+1$ dimensional sigma models, motivated by topological Wess-Zumino-Witten terms which describe the winding number of instanton solutions in three dimensional sigma models. This is analogous to the relation between three dimensional Chern-Simons terms and four dimensional instantons in self-dual Yang-Mills theory. Indeed, torsion sigma models in $2+1$ dimensions with Lie group targets were later found to arise from the chiral field form of self-dual Yang-Mills theory under dimensional reductions 29, 30, 31]. As important motivation, such reductions yield integrable chiral field equations that possess many of the same integrability features in $2+1$ dimensions as are known for chiral models in $1+1$ dimensions [32]. These features geometrically stem from tuning the torsion in the connection on the Lie group target space so as to flatten its generalized Riemannian curvature [28]. Torsion more generally is of interest in the analytical study of the Cauchy problem for sigma models in $2+1$ dimensions 33], since this is the critical dimension in which blow-up may occur for solutions with smooth initial data of large energy [34].

The torsion gauge theories described in this paper will be geometrical generalizations of the dual form of general $2+1$ dimensional torsion chiral models. In particular, as a main new result, torsion will be shown to be consistent with a Yang-Mills interaction of 1-form potentials in $2+1$ spacetime dimensions provided a Chern-Simons mass term is included in 
the full theory. Because the introduction of a skew tensor needed to support the torsion interaction breaks Lorentz covariance at each point in spacetime, the field equations in these new torsion gauge theories have a semi-relativistic form resembling that of $2+1$ dimensional torsion chiral models [31].

To begin, a strengthened geometrical version of the field-theoretic approach to gauge theory deformations developed in the course of earlier work [6, 14, 16] will be summarized in Sec. II] The approach given here simplifies the steps for finding deformation terms up to second order and clearly identifies the obstructions (integrability conditions) that must be satisfied on 1st order deformations for existence of a deformation to higher orders. In particular, it is shown how the Noether current and commutator associated with the 1st order deformed gauge symmetries explicitly determine the quadratic deformation terms in both the field equation and the gauge symmetry. This enables finding all obstructions, which is a key contribution. As further main results of this comprehensive approach, firstly, a simple general quasilinear form for the deformation terms is shown to be required by preserving the number of initial-data and gauge degrees of freedom order by order. Secondly, a remarkable universal geometrical structure derived in terms of a covariant derivative operator associated with the gauge symmetry, along with a nonlinear field strength connected to the curvature of this covariant derivative, is shown to be common to a large class of deformations. The scope of these results is quite general and encompasses the gauge theory examples discussed earlier.

In Sec. III a generalization of nonabelian Yang-Mills Chern-Simons theory incorporating a Freedman-Townsend interaction without torsion is constructed by a deformation analysis of linear abelian Yang-Mills theory with a Chern-Simons term for 1-form potentials in three spacetime dimensions. Geometrical aspects of this generalization are highlighted and shown to display a duality with a principal chiral field theory that is coupled in a novel manner to nonabelian Chern-Simons theory with a Proca mass term. The torsion generalization of three-dimensional nonabelian Yang-Mills Chern-Simons gauge theory is then derived in Sec. IV] The derivation applies a deformation analysis to linear abelian Yang-Mills ChernSimons theory with the inclusion of torsion based on the dual form of torsion chiral models for abelian Lie group targets. Field-theoretic and geometric features of the resulting nonlinear gauge theories with torsion are discussed. In Sec. $\nabla$ an extension of torsion to the gravity-like generalization of Yang-Mills theory is obtained by a similar derivation. 
Concluding remarks are made in Sec. VI] Throughout, notation follows that of Ref. [16].

\section{DEFORMATION METHOD}

For the purpose of a general deformation theory, all gauge theories of primary interest in mathematics and physics can be formulated in common by gauge field variables taken to be vector-valued $p$-form potentials in $d$ dimensions, with $1 \leq p \leq d-2$, for some appropriate internal vector space $\mathbb{V}$. Furthermore, these field variables can be chosen such that the field equations have a zero potential as a solution, and also such that the gauge symmetries when linearized around this solution have the form of a set of $U(1)$ gauge transformations involving an arbitrary vector-valued $p-1$-form as the parameter.

For example, the gauge field variable in $d$-dimensional Yang-Mills theory is a connection 1-form represented by a $p=1$ potential $A_{\mu}^{a}$ that takes values in the Lie algebra of the YangMills gauge group e.g. $\mathbb{V} \simeq S U(2)$. As discussed in the introduction, a linearization about a flat connection $A_{\mu}^{a}=0$ yields the abelian Yang-Mills gauge symmetry, given by $\delta_{\zeta} A_{\mu}^{a}=\partial_{\mu} \zeta^{a}$ for a Lie-algebra valued arbitrary function $(0-$ form $) \zeta^{a}$ on spacetime. Freedman-Townsend theory has a similar linearization, involving a $p=d-2$ potential $B_{\mu_{1} \cdots \mu_{d-2}}^{a}$. As another example, $d$-dimensional gravitational theories are accommodated by using a vielbein (frame) formulation, with the gauge field variable chosen to be the deviation from a flat vielbein associated with a Minkowski space solution of the gravitational field equations, namely $h_{\mu}^{a}=e_{\mu}^{a}-\sigma_{\mu}^{a}$ where $e_{\mu}^{a}$ and $\sigma_{\mu}^{a}$ are respectively the curved and flat spacetime vielbeins. Thus, $h_{\mu}^{a}$ is a $p=1$ potential with values in a $d$-dimensional internal Minkowski space $\mathbb{V} \simeq\left(\mathbb{R}^{d}, \eta_{a b}\right)$. Note the gauge symmetry on the vielbein $e_{\mu}^{a}$ corresponding to diffeomorphism invariance is simply a Lie derivative with respect to an arbitrary vector field $\zeta^{\mu}$ on spacetime. From the discussion in the introduction, a linearization around flat spacetime $h_{\mu}^{a}=0$ yields an abelian graviton gauge symmetry that takes the form of a linearized Lie derivative $\delta_{\zeta} h_{\mu}^{a}=\partial_{\mu} \zeta^{a}$ where $\zeta^{a}=\zeta^{\mu} \sigma_{\mu}^{a}$ is a Minkowski-space valued arbitrary function (0-form) on spacetime, representing the components of the vector field $\zeta^{\mu}$ in the flat vielbein. This gauge invariance in the linearized theory is of the same differential type as in linearized Yang-Mills theory. The only essential difference in the field variables of these two linear abelian theories is the presence of an auxiliary (extra) gauge freedom coming from linearization of the local Lorentz

rotation transformations on the vielbein $e_{\mu}^{a}$, which yields $\delta_{\chi} h_{\mu}^{a}=\eta^{a c} \sigma_{\mu}^{b} \chi_{b c}$ where $\chi_{b c}=-\chi_{c b}$ 
is an arbitrary antisymmetric matrix function on $M$.

For generalizations such as multi-graviton theories, the vector space $\mathbb{V}$ is just enlarged 14, 15] by a tensor product with some kind of internal algebra $\mathfrak{g}$; and for the gravitylike generalization of Yang-Mills theory, $\mathbb{V}$ is any Lie subalgebra of the isometry group admitted by the spacetime metric [27]. In addition, supersymmetric gauge theories such as supergravity are accommodated by enlarging $\mathbb{V}$ as a direct sum to include an appropriate spinor space [15].

In general it will be convenient to write the gauge field variables and their derivatives in local coordinates on spacetime and assume the underlying spacetime manifold is equipped with a fixed metric $g_{\mu \nu}$ and volume form $\epsilon_{\boldsymbol{\mu}_{d}}$ for which the coordinate derivative $\partial_{\alpha}$ is metriccompatible, $\partial_{\alpha} g_{\mu \nu}=0, \partial_{\alpha} \epsilon_{\mu_{1} \cdots \mu_{d}}=0$. As in all the preceding examples, for typical gauge theories the geometrical content is independent of this background spacetime structure.

For the sequel, a multi-index notation will be used to denote collections of $k \geq 1$ spacetime indices $\mu_{1} \cdots \mu_{k}$ as $\boldsymbol{\mu}_{k}$; index symmetrization and antisymmetrization will be denoted by $(\cdots)$ and $[\cdots]$ as usual.

\section{A. Linear abelian gauge theory}

So consider a linear abelian gauge theory on a $d$-dimensional spacetime manifold $M$ using vector-valued $p$-form potentials $\phi_{\boldsymbol{\mu}_{p}}^{a}$ as field variables whose differential gauge invariance is given by $\phi_{\boldsymbol{\mu}_{p}}^{a} \rightarrow \phi_{\boldsymbol{\mu}_{p}}^{a}+\partial_{\left[\mu_{p}\right.} \zeta_{\left.\boldsymbol{\mu}_{p-1}\right]}^{a}$ where $\zeta_{\boldsymbol{\mu}_{p-1}}^{a}$ is vector-valued arbitrary $p-1$-form on $M$. The corresponding abelian gauge symmetry for the theory is given by the infinitesimal field variation

$$
\delta_{\zeta}^{(0)} \phi_{\boldsymbol{\mu}_{p}}^{a}=\partial_{\left[\mu_{p}\right.} \zeta_{\left.\boldsymbol{\mu}_{p-1}\right]}^{a}
$$

The theory is specified by giving a quadratic Lagrangian top-form $\stackrel{(2)}{L}_{\boldsymbol{\mu}_{d}}$ that is locally constructed from the fields $\phi$ and their derivatives $\partial^{k} \phi$, along with a fixed spacetime metric $g_{\mu \nu}$ and volume form $\epsilon_{\boldsymbol{\mu}_{d}}$ on $M$, an inner product $k_{a b}$ fixed on $\mathbb{V}$, as well as any extra structure which may be available on $M$ or $\mathbb{V}$ (for example, a flat vielbein). Gauge invariance is expressed by the curl condition

$$
{\stackrel{(0)(2)}{\delta_{\zeta}}}_{\boldsymbol{\mu}_{d}}=\partial_{\left[\mu_{d}\right.} \stackrel{(1)}{\Theta}_{\left.\boldsymbol{\mu}_{d-1}\right]}
$$


for some locally constructed $d-1$-form $\stackrel{(1)}{\Theta}_{\boldsymbol{\mu}_{d-1}}$. If $\zeta$ is of compact support on $M$ then the action functional $\stackrel{(2)}{S}[\phi]=\int_{M} \stackrel{(2)}{L} \boldsymbol{\mu}_{d}$ is gauge invariant since $\stackrel{(0)(2)}{\delta}_{\zeta}^{S}[\phi]=\int_{M} \partial_{\left[\mu_{d}\right.} \stackrel{(1)}{\Theta}{ }_{\left.\boldsymbol{\mu}_{d-1}\right]}=0$ vanishes by Stokes' theorem. Equivalently, under the abelian gauge symmetry, the scalar Lagrangian $* \stackrel{(2)}{L}=\frac{1}{d !} \epsilon{\stackrel{\boldsymbol{\mu}_{d}}{\stackrel{(2)}{L}}}_{\boldsymbol{\mu}_{d}}$ varies into a divergence $\stackrel{(0)}{\zeta}_{\zeta}^{*} \stackrel{(2)}{L}=\frac{1}{d} \partial_{\mu} * \stackrel{(1)}{\Theta}^{\mu}$ where $* \stackrel{(1)}{\Theta}^{\mu}=\frac{1}{(d-1) !} \epsilon^{\mu \boldsymbol{\mu}_{d-1}} \stackrel{(1)}{\Theta} \boldsymbol{\mu}_{d-1}$.

The stationary points of the action functional yield the linear field equation $\stackrel{(1)}{E_{a}^{\boldsymbol{\mu}_{p}}}=0$ on $\phi$. Gauge invariance of the theory implies this field equation depends on $\phi$ only through the abelian field strength $p+1$-forms

$$
\Phi_{\boldsymbol{\mu}_{p+1}}^{a}=\partial_{\left[\mu_{p+1}\right.} \phi_{\left.\boldsymbol{\mu}_{p}\right]}^{a}
$$

and their derivatives.

In typical theories, the linear field equation is at most second order in derivatives and thus has the form

$$
\stackrel{(1)}{E_{a}^{\boldsymbol{\mu}_{p}}}=q_{a b}^{\boldsymbol{\mu}_{p} \boldsymbol{\nu}_{p+1} \alpha} \partial_{\alpha} \Phi_{\boldsymbol{\nu}_{p+1}}^{b}+p_{a b}^{\boldsymbol{\mu}_{p} \boldsymbol{\nu}_{p+1}} \Phi_{\boldsymbol{\nu}_{p+1}}^{b}
$$

where the coefficients $q, p$ depend only on the structure available on $M$ and $\mathbb{V}$. Correspondingly, the Lagrangian $\stackrel{(2)}{L}_{\boldsymbol{\mu}_{d}}$ is at most first order in derivatives, to within a curl.

\section{B. Preliminaries}

A precise mathematical setting for writing down deformation terms and analyzing the deformation determining equations is provided by the field configuration space $\mathcal{S}=\left\{\phi_{\boldsymbol{\mu}_{p}}^{a}(x)\right\}$, defined as the set of all smooth sections of the vector bundle of $\mathbb{V}$-valued $p$-forms on $M$. Associated with $\mathcal{S}$ is the jet space formally defined using local coordinates

$$
J^{(\infty)}(\mathcal{S})=\left(x, \phi_{\boldsymbol{\mu}_{p}}^{a}, \partial_{\nu_{1}} \phi_{\boldsymbol{\mu}_{p}}^{a}, \partial_{\nu_{1}} \partial_{\nu_{2}} \phi_{\boldsymbol{\mu}_{p}}^{a}, \ldots\right)
$$

where $x$ represents a point in $M ; \phi_{\boldsymbol{\mu}_{p}}^{a}$ represents the value of the $p$-form field $\phi(x)$ at $x$; $\partial_{\boldsymbol{\nu}_{k}}^{k} \phi_{\boldsymbol{\mu}_{p}}^{a}$ represents the values of the $k$ th order derivatives of the $p$-form field $\partial^{k} \phi(x)$ at $x$ (in multi-index notation). Note the action of derivatives on these coordinates in $J^{(\infty)}(\mathcal{S})$ is given by the total derivative operator

$$
\partial_{\nu}=\frac{\partial}{\partial x^{\nu}}+\sum_{k \geq 0} \partial_{\nu} \partial_{\nu_{k}}^{k} \phi_{\boldsymbol{\mu}_{p}}^{a} \frac{\partial}{\partial \partial_{\boldsymbol{\nu}_{k}}^{k} \phi_{\boldsymbol{\mu}_{p}}^{a}}
$$

where $\partial^{k} \phi$ for $k=0$ stands for $\phi$. This setting makes clear what will be meant by locally constructed deformation terms, and it allows the use of tools of variational calculus [6, 35, 36] 
that will be relevant for formulating gauge symmetries, field equations, and the condition of gauge invariance in a general deformation theory.

Geometrically, a field variation $\delta \phi_{\boldsymbol{\mu}_{p}}^{a}$ is a vector field on $\mathcal{S}$. Corresponding vector fields on the jet space $J^{(\infty)}(\mathcal{S})$ that involve no motion on the spacetime coordinates $x^{\mu}$ and that preserve the derivative relations among the field coordinates represent field variations that are locally constructed from the fields and their derivatives as well as from any structure available on $M$ and $\mathbb{V}$. Given such a field variation, there is a natural Lie derivative operator $\mathcal{L}_{\delta \phi}$ defined as follows. On scalar functions $f$ on $J^{(\infty)}(\mathcal{S})$, it acts as a total variation

$$
\mathcal{L}_{\delta \phi} f=\delta f=\sum_{k \geq 0} \frac{\partial f}{\partial \partial_{\boldsymbol{\nu}_{k}}^{k} \phi_{\boldsymbol{\mu}_{p}}^{a}} \partial_{\boldsymbol{\nu}_{k}}^{k} \delta \phi_{\boldsymbol{\mu}_{p}}^{a}
$$

For covector functions $f_{a}^{\boldsymbol{\mu}_{p}}$ on $J^{(\infty)}(\mathcal{S})$, the Lie derivative action is given by

$$
\mathcal{L}_{\delta \phi} f_{a}^{\boldsymbol{\mu}_{p}}=\sum_{k \geq 0}\left(\frac{\partial f_{a}^{\boldsymbol{\mu}_{p}}}{\partial \partial_{\boldsymbol{\alpha}_{k}}^{k} \phi_{\boldsymbol{\nu}_{p}}^{b}} \partial_{\boldsymbol{\alpha}_{k}}^{k} \delta \phi_{\boldsymbol{\nu}_{p}}^{b}+(-1)^{k} \partial_{\boldsymbol{\alpha}_{k}}^{k}\left(\frac{\partial \delta \phi_{\boldsymbol{\nu}_{p}}^{b}}{\partial \partial_{\boldsymbol{\alpha}_{k}}^{k} \phi_{a}^{\boldsymbol{\mu}_{p}}} f_{b}^{\boldsymbol{\nu}_{p}}\right)\right) .
$$

This action extends via the Leibniz rule to vector functions and, more generally, any tensor functions on $J^{(\infty)}(\mathcal{S})$. The Lie derivative operator on scalar and covector functions will be central here to the formulation of deformation determining equations.

There are two useful identities that hold for the Lie derivative operator. Firstly, Lie derivatives with respect to any locally constructed field variations $\delta_{1} \phi$ and $\delta_{2} \phi$ satisfy the familiar commutation relation

$$
\left[\mathcal{L}_{\delta_{1} \phi}, \mathcal{L}_{\delta_{2} \phi}\right]=\mathcal{L}_{\left[\delta_{1} \phi, \delta_{2} \phi\right]}
$$

where $\left[\delta_{1} \phi, \delta_{2} \phi\right]=\delta_{\delta_{1} \phi} \delta_{2} \phi-\left(\zeta_{1} \leftrightarrow \zeta_{2}\right)$ defines the commutator of the field variations. Secondly, the Jacobi relation is satisfied, $\left[\mathcal{L}_{\delta_{1} \phi},\left[\mathcal{L}_{\delta_{2} \phi}, \mathcal{L}_{\delta_{3} \phi}\right]\right]-$ cyclic terms $=0$, since for any three locally constructed field variations,

$$
\left[\delta_{1} \phi,\left[\delta_{2} \phi, \delta_{3} \phi\right]\right]-\text { cyclic terms }=0
$$

holds identically. These properties are direct consequences of the representation of field variations as vector fields on jet space.

Another important variational operator will be the Euler-Lagrange operator acting on scalar functions $f$ on $J^{(\infty)}(\mathcal{S})$ by

$$
\mathrm{E}_{\phi_{\boldsymbol{\mu}_{p}}^{a}}(f)=\sum_{k \geq 0}(-1)^{k} \partial_{\boldsymbol{\nu}_{k}}^{k} \frac{\partial f}{\partial \partial_{\boldsymbol{\nu}_{k}}^{k} \phi_{\boldsymbol{\mu}_{p}}^{a}} .
$$


This operator takes functions $f$ into covector functions $f_{a}^{\boldsymbol{\mu}_{p}}=\mathrm{E}_{\phi_{\boldsymbol{\mu}_{p}}^{a}}(f)$ and has the property that it annihilates a locally constructed function $f$ if and only if on $J^{(\infty)}(\mathcal{S})$ the dual topform function is a curl, $* f_{\boldsymbol{\mu}_{d}}=\partial_{\left[\mu_{d}\right.} \Theta_{\left.\boldsymbol{\mu}_{d-1}\right]}$, for some locally constructed $d$ - 1-form function $\Theta_{\boldsymbol{\mu}_{d-1}}$.

The Euler-Lagrange and Lie derivative operators are related through integration by parts. In particular, for scalar functions $f$ on $J^{(\infty)}(\mathcal{S})$, repeated integration by parts on a Lie derivative with respect to any locally constructed vector field $\delta \phi$ on $J^{(\infty)}(\mathcal{S})$ yields

$$
\mathcal{L}_{\delta \phi} f=\delta \phi_{\boldsymbol{\nu}_{p}}^{a} f_{a}^{\boldsymbol{\nu}_{p}}+\partial_{\mu} \Upsilon^{\mu}(f ; \delta \phi), \quad f_{a}^{\boldsymbol{\nu}_{p}}=\mathrm{E}_{\phi_{\boldsymbol{\nu}_{p}}^{a}}(f)
$$

where

$$
\Upsilon^{\mu}(f ; \delta \phi)=\sum_{k \geq 0}\left(\partial_{\boldsymbol{\alpha}_{k}}^{k} \delta \phi_{\boldsymbol{\nu}_{p}}^{a}\right) \mathrm{E}_{\partial_{\mu \boldsymbol{\alpha}_{k}}^{k+1} \phi_{\nu_{p}}^{a}}(f)
$$

\section{Determining equations for nonlinear deformations}

A deformation of a linear abelian gauge theory for fields $\phi$ consists of adding linear and higher power terms to the abelian gauge symmetry (2.1),

$$
\delta_{\zeta} \phi_{\boldsymbol{\mu}_{p}}^{a}=\stackrel{(0)}{\delta}_{\zeta} \phi_{\boldsymbol{\mu}_{p}}^{a}+\stackrel{(1)}{\delta}_{\zeta}^{a} \boldsymbol{\mu}_{p}+\cdots
$$

while simultaneously adding quadratic and higher power terms to the linear field equation (2.4),

$$
E_{a}^{\boldsymbol{\mu}_{p}}=\stackrel{(1)}{E_{a}^{\boldsymbol{\mu}_{p}}}+\stackrel{(2)}{E_{a}^{\boldsymbol{\mu}_{p}}}+\cdots
$$

such that there exists a top-form Lagrangian

$$
L_{\boldsymbol{\mu}_{d}}=\stackrel{(2)}{L}_{\boldsymbol{\mu}_{d}}+\stackrel{(3)}{L}_{\boldsymbol{\mu}_{d}}+\cdots
$$

required to be gauge invariant to within a curl. Here the deformation terms in the field equation and gauge symmetry are to be locally constructed from powers of the fields $\phi$ and their derivatives $\partial^{k} \phi$ up to some finite differential order, with coefficients allowed to depend on the spacetime coordinates $x^{\mu}$, the spacetime metric $g_{\mu \nu}$ and volume form $\epsilon_{\boldsymbol{\mu}_{d}}$, the internal vector-space inner product $k_{a b}$, and any other available structure on $M$ or $\mathbb{V}$. As well, the deformation terms in the gauge symmetry are to be linear in the gauge parameter $\zeta$ and its derivatives $\partial^{j} \zeta$ to a finite differential order. Thus, the expressions for $\delta_{\zeta} \phi_{\boldsymbol{\mu}_{p}}^{a}$ and $E_{a}^{\boldsymbol{\mu}_{p}}$ will 
be, respectively, vector and covector functions on $J^{(\infty)}(\mathcal{S})$. Natural restrictions on the order of derivatives appearing in these expressions will be addressed shortly (see proposition 2.4).

The condition of gauge invariance is stated by

$$
\delta_{\zeta} L \boldsymbol{\mu}_{d}=\stackrel{(0)(2)}{\delta}_{\zeta}^{L}{ }_{\boldsymbol{\mu}_{d}}+\stackrel{(1)(2)}{\delta}_{\zeta}^{L}{ }_{\boldsymbol{\mu}_{d}}+\stackrel{(0)(3)}{\delta}_{\zeta}^{L} \boldsymbol{\mu}_{d}+\cdots=\partial_{\left[\mu_{d}\right.} \stackrel{(1)}{\Theta}_{\left.\boldsymbol{\mu}_{d-1}\right]}
$$

holding for some $d$-1-form function $\stackrel{(1)}{\Theta}_{\mu_{d-1}}$ on $J^{(\infty)}(\mathcal{S})$, where the Lagrangian (2.16) is related to the field equation (2.15) through the Euler-Lagrange operator,

$$
\mathrm{E}_{\phi_{\boldsymbol{\mu}_{p}}^{a}}(\stackrel{(k+1)}{L})=\stackrel{(k)}{E_{a}^{\boldsymbol{\mu}_{p}}}
$$

Necessary and sufficient conditions for $\stackrel{(k)}{E}$ to arise as an Euler-Lagrange equation are that the Frechet derivative of $\stackrel{(k)}{E}$ must be a self-adjoint operator [35, 37]. These conditions can be shown to determine

$$
\stackrel{(k+1)}{L}_{\boldsymbol{\mu}_{d}}=-\frac{1}{k+1} \epsilon_{\boldsymbol{\mu}_{d}} \stackrel{(k)}{E_{b}^{\nu_{p}}} \phi_{\boldsymbol{\nu}_{p}}^{b}
$$

to within a curl. In terms of the Euler-Lagrange operator, the condition of gauge invariance is expressed through the integration by parts identity (2.12) by the equation

$$
\mathrm{E}_{\phi_{\boldsymbol{\mu}_{p}}^{a}}\left(E_{b}^{\boldsymbol{\nu}_{p}} \delta_{\zeta} \phi_{\boldsymbol{\nu}_{p}}^{b}\right)=0
$$

on $J^{(\infty)}(\mathcal{S})$. This constitutes the determining equation for all allowed deformations of the linear abelian gauge theory (2.1) and (2.4).

The deformation determining equation (2.20) can be reformulated more usefully and geometrically as Lie derivative equations, as shown by results in $[6]$.

Theorem 2.1: Gauge invariance holds iff the Lie derivative of the field equation with respect to the gauge symmetry vanishes

$$
\mathcal{L}_{\delta_{\zeta} \phi} E_{a}^{\mu_{p}}=0
$$

This equation asserts that the gauge symmetry for each parameter is a vector field tangent to the surface in $J^{(\infty)}(\mathcal{S})$ corresponding to the field equation (and all its derivatives), $J^{(\infty)}(\mathcal{S}) \quad(\bmod E=0) \equiv J^{(\infty)}(\mathcal{E}) \subset J^{(\infty)}(\mathcal{S})$. Due to invariance of the action functional, the commutators of these vector fields for all parameters have the same tangency property.

Theorem 2.2: Gauge invariance holds only if the Lie derivative of the field equation with respect to the gauge symmetry commutators vanishes

$$
\mathcal{L}_{\left[\delta_{\zeta_{1} \phi} \phi, \delta_{\zeta_{2}} \phi\right]} E_{a}^{\boldsymbol{\mu}_{p}}=0 .
$$


It is important to remark that here no conditions are assumed or required on the possibilities allowed for the form of the commutators of the deformed gauge symmetries. Nevertheless, when the gauge symmetries are restricted to the solution space of the deformed field equations, closure of the commutators will be seen to arise order by order, stemming from the fact that the abelian gauge symmetries generate all of the differential gauge invariance present in the solution space of the linear field equations (i.e. modulo any auxiliary gauge freedom). Any deformation therefore will automatically determine an associated infinitesimal gauge group structure. As will be stated in precise form later (see also Ref. 38]), the commutators characterizing this group structure may involve local structure functions that depend on the fields $\phi$ (and their derivatives) and may fail to close other than when the fields satisfy $E=0$.

A related remark is that gauge invariance as expressed by the Lie derivative equation (2.21) implies the deformed gauge symmetry $\delta_{\zeta} \phi$ formally generates an infinitesimal symmetry group of the deformed field equation, since

$$
\delta_{\zeta} E_{a}^{\boldsymbol{\mu}_{p}}=0 \text { when } \phi \text { satisfies } E=0
$$

Geometrically, this equation is precisely the condition for the associated vector field $\delta_{\zeta} \phi$ in $J^{(\infty)}(\mathcal{S})$ to lie tangent to the solution space surface $J^{(\infty)}(\mathcal{E})$. (This general notion of symmetries defined as locally constructed tangent vector fields to $J^{(\infty)}(\mathcal{E})$ comprises classical point symmetries as well as generalized or higher order symmetries of a field equation $E=0$; see Refs. [35, 37, 39].) However, it is worthwhile to emphasize here that the symmetry determining equation (2.23) is strictly weaker than the Lie derivative equation (2.21), as not every symmetry of an Euler-Lagrange field equation will necessarily generate an invariance of its action functional; for example, symmetries that scale the coordinates of $J^{(\infty)}(\mathcal{S})$ typically leave invariant the Lagrangian only in certain spacetime dimensions.

There is a related formulation of gauge invariance from this point of view, which is also useful. Introduce the gauge parameter space $\mathcal{P}=\left\{\zeta_{\boldsymbol{\mu}_{p-1}(x)}^{a}\right\}$ defined as the vector bundle of all smooth $\mathbb{V}$-valued $p-1$-forms on $M$. The gauge symmetry (2.14) then can be viewed as a linear differential operator

$$
\delta_{\zeta} \phi_{\boldsymbol{\mu}_{p}}^{a}=D_{\phi}(\zeta)_{\boldsymbol{\mu}_{p}}^{a}
$$

from $\mathcal{P}$ to $\mathcal{S}$ that is locally constructed from $\phi$, derivatives of $\phi$, and any structure available on $M$ and $\mathbb{V}$. Note via integration by parts, this operator $D_{\phi}$ has a formal adjoint $D_{\phi}^{*}$ that 
acts as a linear differential operator from $\mathcal{S}^{*}$ into $\mathcal{P}^{*}$ (i.e. the dual vector bundles of $\mathcal{S}$ and $\mathcal{P}$ ). Now, an application of the Euler-Lagrange operator with respect to $\zeta_{\boldsymbol{\nu}_{p-1}}^{b}$ in the statement of gauge invariance (2.17) gives a Noether divergence identity

$$
D_{\phi}^{*}(E)_{b}^{\boldsymbol{\nu}_{p-1}}=0
$$

Conversely, contraction of $\zeta_{\boldsymbol{\nu}_{p-1}}^{b}$ onto this identity followed by use of the Euler-Lagrange relation (2.18) along with repeated integration by parts then gives back equation (2.17).

Proposition 2.3: Gauge invariance holds iff the field equation satisfies the Noether identity (2.25) derived from the gauge symmetry.

Now to proceed, an expansion of the Lie derivative equations (2.21) and (2.22) in powers of the field coordinates in $J^{(\infty)}(\mathcal{S})$ gives a hierarchy of determining equations whose solutions yield all allowed deformation terms for the field equation and gauge symmetry. Before looking at this hierarchy, it is important to consider the order of derivatives on $\phi$ and $\zeta$ allowed in the deformation terms.

First, a natural requirement is that the number of dynamical degrees of freedom of the fields $\phi$ in the linear abelian theory should be preserved order by order in a nonlinear deformation. Otherwise, severe consistency conditions could arise on solutions of the field equation, and the deformed theory would not be physically or mathematically satisfactory. (These degrees of freedom can be identified as the number of initial-data functions modulo the gauge symmetry freedom, provided that the linear field equation is a well-posed system of PDEs after suitable gauge constraints have been imposed on the fields.) With this condition, for the typical situation in which the linear field equation is a system of second order PDEs, the most general possible form then allowed for deformed field equations will be a quasilinear second order PDE system, namely, highest derivatives are of second order while the coefficients of these terms depend on no higher than first order derivatives,

$$
\stackrel{(k)}{E}{ }_{a}^{\boldsymbol{\mu}_{p}}=\stackrel{(k-1)}{Q} \underset{a b}{\boldsymbol{\mu}_{p} \boldsymbol{\nu}_{p} \alpha \beta} \partial_{\alpha} \partial_{\beta} \phi_{\boldsymbol{\nu}_{p}}^{b}+\stackrel{(k-1)}{P} \underset{a b}{\boldsymbol{\mu}_{p} \boldsymbol{\nu}_{p} \alpha} \partial_{\alpha} \phi_{\boldsymbol{\nu}_{p}}^{b}+\stackrel{(k-1)}{R} \underset{a b}{\boldsymbol{\mu}_{p} \boldsymbol{\nu}_{p}} \phi_{\boldsymbol{\nu}_{p}}^{b}
$$

Here, for later notational ease, the lower derivative terms have been written in a factored form where $\stackrel{(k-1)}{R}$ contains no derivatives of $\phi$ and $\stackrel{(k-1)}{P}$ contains no higher than first derivatives of $\phi$. Note that the form of the linear field equation gives

$$
\stackrel{(0)}{Q}{ }_{a b}^{\boldsymbol{\mu}_{p} \boldsymbol{\nu}_{p} \nu_{p+1} \alpha}=q_{a b}^{\boldsymbol{\mu}_{p} \boldsymbol{\nu}_{p+1} \alpha}, \quad \stackrel{(0)}{P} \underset{a b}{\boldsymbol{\mu}_{p} \boldsymbol{\nu}_{p} \nu_{p+1}}=p_{a b}^{\boldsymbol{\mu}_{p} \boldsymbol{\nu}_{p+1}}, \quad \stackrel{(0)}{R} \underset{a b}{\boldsymbol{\mu}_{p} \boldsymbol{\nu}_{p}}=0
$$


Next, as a consequence of the quasilinear second-order derivative form sought for the deformed field equations, a bound arises on the order of derivatives allowed in the deformed gauge symmetry through the Noether divergence identity (2.25), as follows. The deformed gauge symmetry has the form of a linear differential operator on $\zeta$, with coefficients $U_{\boldsymbol{\mu}_{p} b}^{a \nu_{p-1}}$ of $\zeta_{\boldsymbol{\nu}_{p-1}}^{b}$ and $V_{\boldsymbol{\mu}_{p} b}^{a \boldsymbol{\nu}_{p-1} \boldsymbol{\alpha}_{j}}$ of $\partial_{\boldsymbol{\alpha}_{j}}^{j} \zeta_{\boldsymbol{\nu}_{p-1}}^{b}$ for $1 \leq j$, up to some highest order $j_{\text {max }}$ in derivatives. Its associated Noether identity on the deformed field equation is then given by

$$
0=D_{\phi}^{*}(E)_{a}^{\boldsymbol{\mu}_{p-1}}=E_{b}^{\boldsymbol{\nu}_{p}} U_{\boldsymbol{\nu}_{p} a}^{b \boldsymbol{\mu}_{p-1}}+\sum_{1 \leq j \leq j_{\max }}(-1)^{j} \partial_{\boldsymbol{\alpha}_{j}^{j}}\left(E_{b}^{\boldsymbol{\nu}_{p}} V_{\boldsymbol{\nu}_{p} a}^{b \boldsymbol{\mu}_{p-1} \boldsymbol{\alpha}_{j}}\right)
$$

When this identity is expanded in powers of the field coordinates in $J^{(\infty)}(\mathcal{S})$, taking into account the form of the abelian gauge symmetry, it gives divergence equations of the form

$$
\partial_{\mu_{p}} \stackrel{(k)}{E}_{a}^{\boldsymbol{\mu}_{p}}=\sum_{1 \leq i \leq k-1}\left(\stackrel{(i)}{E}_{b}^{\boldsymbol{\nu}_{p}} \stackrel{(k-i)}{U} \boldsymbol{\mu}_{\boldsymbol{\mu}_{p-1}}+\sum_{1 \leq j \leq j_{\max }}(-1)^{j} \partial_{\boldsymbol{\alpha}_{j}}^{j}\left(\stackrel{(i)}{E}_{b}^{\boldsymbol{\nu}_{p}} \stackrel{(k-i)}{V}_{\boldsymbol{\nu}_{p} \boldsymbol{\mu}_{p-1} \boldsymbol{\alpha}_{j}}\right)\right)
$$

for each $k \geq 2$. In general, as $k$ increases, these equations imply an unbounded escalation in the order of derivatives on $\phi$ in the deformation terms $\stackrel{(k)}{E}$ compared with $\stackrel{(1)}{E}$, unless $\stackrel{(k)}{V}$ vanishes for $j \geq 2$. Moreover, for a balance to hold in the order of derivatives appearing on both sides of the divergence equations under the condition that $\stackrel{(k)}{E}$ is quasilinear second order in derivatives of $\phi, \stackrel{(k)}{V}$ for $j=1$ should contain no second or higher order derivatives of $\phi$ while $\stackrel{(k)}{U}$ should contain second derivatives of $\phi$ at most linearly.

Therefore, given a general quasilinear second-order derivative form for the deformed field equation, the most general possible form allowed for the deformed gauge symmetry will be a first-order linear differential operator on $\zeta$

$$
\stackrel{(k)}{\delta}_{\zeta} \phi_{\boldsymbol{\mu}_{p}}^{a}=\stackrel{(k)}{U}_{\boldsymbol{\mu}_{p} b}^{a \boldsymbol{\nu}_{p-1}} \zeta_{\boldsymbol{\nu}_{p-1}}^{b}+\stackrel{(k)}{V}_{\boldsymbol{\mu}_{p} b}^{a \boldsymbol{\nu}_{p-1} \alpha} \partial_{\alpha} \zeta_{\boldsymbol{\nu}_{p-1}}^{b}
$$

whose coefficients $\stackrel{(k)}{V}$ and $\stackrel{(k)}{U}$ respectively have no dependence on higher than first and second order derivatives of $\phi$, while $\stackrel{(k)}{U}$ is at most linear in second derivatives of $\phi$. Note, from the form of the abelian gauge symmetry, the coefficients at lowest order are given by

$$
\stackrel{(0)}{U_{\boldsymbol{\mu}_{p} b}^{a \boldsymbol{\nu}_{p-1}}}=0, \quad \stackrel{(0)}{V_{\boldsymbol{\mu}_{p} b}^{a \nu_{p-1} \alpha}}=\delta_{b}^{a} \delta_{\boldsymbol{\mu}_{p}}^{\boldsymbol{\nu}_{p-1} \alpha}
$$

However, the divergence equations (2.29) show that, with $j_{\max }=1$, any dependence on first derivatives of $\phi$ in $\stackrel{(k)}{V}$ or second derivatives of $\phi$ in $\stackrel{(k)}{U}$ will lead to an escalating total number of derivatives in $\stackrel{(k)}{E}$ as $k$ increases $[3$, , 14], in which case the deformed field 
equation will be non-polynomial in first (lowest order) derivatives of $\phi$. In particular, count one derivative as a first derivative appearing linearly, two derivatives as a first derivative appearing quadratically or a second derivative appearing linearly, and so on. Now, if $\stackrel{(k)}{E}$ is to be only polynomial in first derivatives (and at most linear in second derivatives), then clearly the number of derivatives on both sides of

$$
\partial_{\alpha}\left(E_{b}^{\boldsymbol{\nu}_{p}} V_{\boldsymbol{\nu}_{p} a}^{b \boldsymbol{\mu}_{p-1}^{\alpha}}\right)=E_{b}^{\boldsymbol{\nu}_{p}} U_{\boldsymbol{\nu}_{p} a}^{b \boldsymbol{\mu}_{p-1}}
$$

will balance only if $\stackrel{(k)}{V}$ contains no derivatives of $\phi$ and $\stackrel{(k)}{U}$ contains first order derivatives of $\phi$ at most linearly and no second order derivatives of $\phi$, for every order $k \geq 1$. Furthermore, at each successive order $k$, this balance in the number of derivatives will imply $\stackrel{(k)}{E}$ should only contain the same number of derivatives as contained in $\stackrel{(1)}{E}$.

Proposition 2.4: With a polynomial restriction on lowest-order derivatives of $\phi$ in the quasilinear form (2.26) sought for the deformed field equation, the most general compatible form for the deformed gauge symmetry will be linear in first derivatives of both $\phi$ and $\zeta$. The deformed field equation, in turn, must be only of a semilinear second-order derivative form, with no derivatives of $\phi$ allowed to appear in the coefficient of the second derivative (highest order) term and at most quadratic dependence on derivatives of $\phi$ allowed for the first derivative (lowest order) term.

Next, the Noether divergence identity (2.32) leads to an interesting relation connecting the gauge symmetry and the field equation. Associated with the deformed gauge symmetry is a rigid symmetry defined by the field variation

$$
\delta_{\left.\zeta\right|_{\text {rigid }}} \phi_{\boldsymbol{\mu}_{p}}^{a}=\left.U_{\boldsymbol{\mu}_{p} b}^{a \nu_{p-1}} \zeta_{\boldsymbol{\nu}_{p-1}}^{b}\right|_{\text {rigid }},\left.\quad \zeta\right|_{\text {rigid }}=\text { const. }
$$

Due to gauge invariance, this field variation leaves the deformed Lagrangian invariant to within a curl,

$$
\delta_{\left.\zeta\right|_{\text {rigid }}} L_{\boldsymbol{\mu}_{d}}=\partial_{\left[\mu_{d}\right.} \Theta_{\left.\boldsymbol{\mu}_{d-1}\right]},
$$

and hence it generates a conserved current of the deformed field equation by Noether's theorem [35, 37]. The current arises from the identity

$$
\begin{aligned}
\mathcal{L}_{\delta_{\left.\zeta\right|_{\text {rigid }}} \phi}(* L) & =\delta_{\left.\zeta\right|_{\text {rigid }}} * L=\frac{1}{d} \partial_{\mu} * \Theta^{\mu} \\
& =E_{a}^{\boldsymbol{\mu}_{p}} \delta_{\left.\zeta\right|_{\text {rigid }}} \phi_{\boldsymbol{\mu}_{p}}^{a}+\partial_{\mu} \Upsilon^{\mu}
\end{aligned}
$$


where $\Upsilon^{\mu}$ is the integration by parts term (2.13) relating the Lie derivative and EulerLagrange operators. This yields

$$
\partial_{\mu} J^{\mu}\left(\left.\zeta\right|_{\text {rigid }}\right)=E_{a}^{\boldsymbol{\mu}_{p}} \delta_{\left.\zeta\right|_{\text {rigid }}} \phi_{\boldsymbol{\mu}_{p}}^{a}, \quad J^{\mu}\left(\left.\zeta\right|_{\text {rigid }}\right)=\frac{1}{d} * \Theta^{\mu}-\Upsilon^{\mu}=J_{b}^{\mu \boldsymbol{\nu}_{p-1}} \zeta_{\boldsymbol{\nu}_{p-1}}^{b},
$$

from which the Noether current $J$ is seen to be conserved on the solution space, $E=0$. Now, a comparison of the conservation equation (2.36) with the divergence identity (2.32) reveals the relation $J^{\prime \mu}\left(\left.\zeta\right|_{\text {rigid }}\right)={J^{\prime}}_{b}^{\mu \boldsymbol{\nu}_{p-1}} \zeta_{\boldsymbol{\nu}_{p-1}}^{b}=\left.E_{a}^{\boldsymbol{\alpha}_{p}} V_{\boldsymbol{\alpha}_{p} b}^{a \boldsymbol{\nu}_{p-1} \mu} \zeta_{\boldsymbol{\nu}_{p-1}}^{b}\right|_{\text {rigid }}$ where the factorized current $J^{\prime}$ is equivalent to $J$ to within a locally constructed, identically divergence-free expression.

Proposition 2.5: The deformed field equation can be written in terms of a rigid Noether current

$$
E_{a}^{\boldsymbol{\alpha}_{p}}=V_{a \boldsymbol{\nu}_{p-1} \mu}^{-1 \boldsymbol{\alpha}_{p} b} J_{b}^{\prime \mu \boldsymbol{\nu}_{p-1}}
$$

which is associated with the deformed gauge symmetry

$$
\partial_{\mu} J_{b}^{\prime \mu \nu_{p-1}}=E_{a}^{\boldsymbol{\alpha}_{p}} U_{\boldsymbol{\alpha}_{p} b}^{a \boldsymbol{\nu}_{p-1}}
$$

where $V^{-1}$ denotes the formal left-inverse of $V$ defined in powers of the field coordinates through equation (2.31).

Finally, deformations that are related by a locally constructed invertible change of field variables

$$
\phi_{\boldsymbol{\mu}_{p}}^{a} \rightarrow \phi_{\boldsymbol{\mu}_{p}}^{\prime a}=\phi_{\boldsymbol{\mu}_{p}}^{a}+\stackrel{(2)}{\prime a}_{\boldsymbol{\mu}_{p}}^{a}+\cdots
$$

or of gauge parameters

$$
\zeta_{\boldsymbol{\mu}_{p-1}}^{a} \rightarrow \zeta^{\prime a} \boldsymbol{\mu}_{p-1}=\zeta_{\boldsymbol{\mu}_{p-1}}^{a}+{\stackrel{(1)}{\zeta^{\prime}}}_{\boldsymbol{\mu}_{p-1}}^{a}+\cdots
$$

will be regarded as equivalent. In order to stay within the general form given for deformation terms (2.26) and (2.30), these transformation terms ${ }^{\prime(k)}{ }_{\boldsymbol{\mu}_{p}}$ and ${ }^{(k-1)}{ }^{\prime}{ }_{\boldsymbol{\mu}_{p-1}}$ for $k \geq 2$ must contain no derivatives of $\phi$, and $\zeta^{\prime}{ }^{\prime}{ }_{\boldsymbol{\mu}_{p-1}}$ must also depend linearly on $\zeta$ (and contain no derivatives of $\zeta)$.

\section{Solving the determining equations}

Steps will now be outlined for how to solve for the deformation terms in an efficient, explicit manner from the hierarchy of determining equations given by theorems 2.1 and 2.2. 
In this hierarchy

$$
\begin{aligned}
& 0 t h: \quad \underset{\delta_{\zeta} \phi}{\mathcal{L}_{(0)}} \stackrel{(1)}{E}=0 \\
& \mathcal{L}_{\delta_{\zeta_{1}} \delta_{\zeta_{2}} \phi} \stackrel{(1)}{E}-\left(\zeta_{1} \leftrightarrow \zeta_{2}\right)=0
\end{aligned}
$$

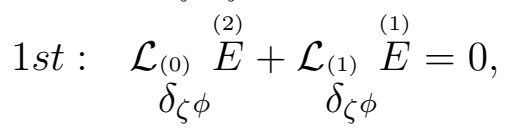

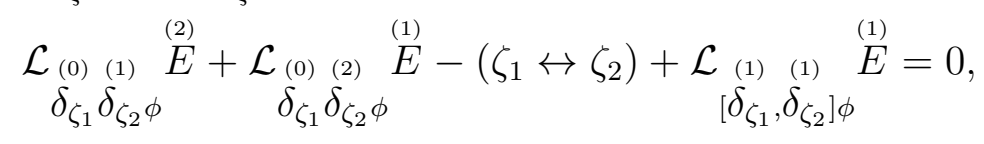

$$
\begin{aligned}
& 2 n d: \quad \ldots
\end{aligned}
$$

observe that the unknowns $\stackrel{(k)}{\delta_{\zeta}} \phi$ and $\stackrel{(k+1)}{E}$ for $k \geq 1$ are partly decoupled since they first enter the equations at $(k-1)$ th and $k$ th orders, respectively. Also, notice the bottom equation (2.41) holds as a gauge-invariance identity on $\stackrel{(0)}{\delta_{\zeta}} \phi$ and $\stackrel{(1)}{E}$ from the given linear abelian theory.

At each order $k \geq 1$, an important feature of the hierarchy is that the unknowns $\stackrel{(k)}{\delta_{\zeta}} \phi$ and $\stackrel{(k+1)}{E}$ occur only through variations $\delta_{\zeta_{2}}^{(0)}\left(\stackrel{(k)}{\delta_{\zeta_{1}}} \phi\right)$ and $\stackrel{(0)(k+1)}{\delta_{\zeta}}$ involving the undeformed gauge symmetry $\stackrel{(0)}{\delta_{\zeta}} \phi$. Such variational expressions can be annihilated in two different ways, leading to integrability conditions in solving for the quadratic and higher power deformation terms. One integrability condition is that an antisymmetrized variation with respect to the undeformed gauge symmetry vanishes, since by commutativity,

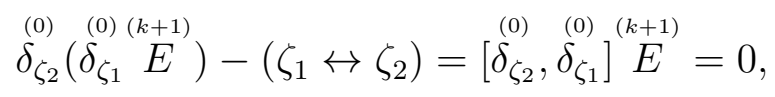

and similarly

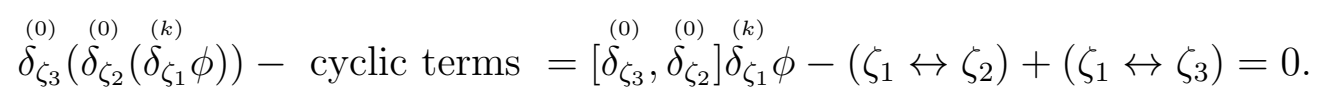

A second integrability condition arises because the undeformed gauge symmetry itself vanishes if its parameter $\zeta$ is taken to be rigid, i.e. constant, so that $\stackrel{(0)}{\delta}_{\left.\zeta\right|_{\text {rigid }}} \phi=0$. Hence, by rigidity,

$$
\left.\stackrel{(0)}{\delta}\right|_{\zeta \text { rigid }} \stackrel{(k+1)}{E}=0
$$

and

$$
\stackrel{(0)}{\delta}_{\left.\zeta_{2}\right|_{\text {rigid }}}\left(\left.\stackrel{(k)}{\delta^{\zeta}} \zeta_{1}\right|_{\text {rigid }} \phi\right)=0
$$


These integrability conditions will be referred to as "commutativity-type" and "rigiditytype". As a main result, because of the decoupled structure of the hierarchy, the commutativity-type integrability condition will turn out to be trivial, as at each order it will hold due to the Jacobi identity for the deformed gauge symmetry and the closure of the associated gauge group, discussed earlier. In contrast, the rigidity-type integrability condition will be central to the deformation analysis.

Connected with the identity (2.41) at the bottom of the hierarchy, the property that the abelian gauge symmetry generates all of the differential gauge invariance in the linear field equation leads to a very useful result.

Lemma 2.6: For any locally constructed field variation $\stackrel{(k)}{\delta} \phi(\zeta)$ depending linearly on at least one arbitrary gauge parameter $\zeta$, the following three conditions are equivalent (provided auxiliary gauge freedom is excluded): (i) It has the form of an undeformed gauge symmetry

$$
\stackrel{(k)}{\delta} \phi_{\boldsymbol{\mu}_{p}}^{a}(\zeta)=\partial_{\left[\mu_{p}\right.}{\stackrel{(k)}{\zeta^{\prime}}}_{\left.\boldsymbol{\mu}_{p-1}\right]}^{a}(\zeta)
$$

whose parameter $\zeta^{\prime}$ depends linearly on $\zeta$ (and its derivatives). (ii) It is identically curl-free

$$
\stackrel{(k)}{\delta} \Phi_{\boldsymbol{\mu}_{p+1}}^{a}=\partial_{\left[\mu_{p+1}\right.} \stackrel{(k)}{\delta} \phi_{\left.\boldsymbol{\mu}_{p}\right]}^{a}(\zeta)=0
$$

(iii) It identically satisfies the undeformed field equation

$$
\left.\stackrel{(k)(1)}{\delta} \stackrel{\boldsymbol{\mu}}{E}_{a}=\stackrel{(1)}{E_{a}^{\boldsymbol{\mu}_{p}}} \stackrel{(k)}{\delta} \phi(\zeta)\right)=0
$$

Moreover, the same equivalences hold whenever $\phi$ satisfies $\stackrel{(1)}{E}(\phi)=0$.

The proof $(i) \Rightarrow(i i) \Rightarrow(i i i)$ is immediate, since $\stackrel{(1)}{E}$ is gauge invariant under $\stackrel{(0)}{\delta_{\zeta}} \phi$. For the converse, $(i i i) \Rightarrow(i i)$ is a consequence of $\stackrel{(0)}{\delta_{\zeta}} \phi$ exhausting all the gauge invariance admitted by $\stackrel{(1)}{E}$ (under the assumption no auxiliary gauge freedom is present), while $(i i) \Rightarrow(i)$ holds because on jet space any closed $p$-form function is exact [36]. These arguments are not difficult to extend to the solution space $\stackrel{(1)}{E}=0$ by means of the jet-space tensor techniques from Ref. [14].

This lemma will be a key tool in the analysis to follow. In examples, the condition of no auxiliary gauge freedom is met by linear abelian Yang-Mills and Freedman-Townsend theories, including abelian Chern-Simons and torsion generalizations, and their extension to other $p$-form fields. The main examples of a linear abelian theory having auxiliary gauge 
freedom are graviton theories. The simplest situation occurs when this auxiliary gauge freedom does not enter into solutions of the determining equations for the deformed field equation and deformed gauge symmetry, order by order. For solutions of that type the deformation terms in the gauge symmetry will satisfy the equivalences stated in the lemma. This is what happens for the deformation of linear abelian graviton theory corresponding to the Einstein gravitational theory and its multi-graviton (algebra-valued) generalizations, in $d \geq 3$ dimensions [13, 14]. In contrast, the exotic parity-violating multi-graviton theories in $d=3,5$ dimensions involve a more complicated situation where the auxiliary gauge freedom from the linear theory is itself deformed nontrivially in the course of solving the determining equations [20].

So, as the simplest case, only those deformations obtained by postulating the lemma to hold independently of any auxiliary gauge freedom in solving the determining equations will be considered hereafter. Note this assumption is trivially met whenever the abelian gauge symmetry exhausts the gauge freedom present in the linear field equation.

To begin the analysis, the first step is to solve the 0th order Lie derivative commutator equation (2.42) for the linear deformation terms in the gauge symmetry

$$
\stackrel{(1)}{\delta_{\zeta}} \phi_{\boldsymbol{\mu}_{p}}^{a}=\stackrel{(1)}{U_{\boldsymbol{\mu}_{p} b}^{a \boldsymbol{\nu}_{p-1}}} \zeta_{\boldsymbol{\nu}_{p-1}}^{b}+\stackrel{(1)}{V_{\boldsymbol{\mu}_{p} b}^{a \nu_{p-1} \alpha}} \partial_{\alpha} \zeta_{\boldsymbol{\nu}_{p-1}}^{b}
$$

Through lemma 2.6, equation (2.42) is equivalent to

$$
0=\partial_{[\nu} \delta_{\zeta_{2}}^{(0)} \delta_{\zeta_{1}}^{(1)} \phi_{\left.\boldsymbol{\mu}_{p}\right]}^{a}-\left(\zeta_{1} \leftrightarrow \zeta_{2}\right)
$$

which expands out to give

$$
\begin{aligned}
& 0=\partial_{[\nu \mid}\left(\sum_{0 \leq k \leq 2} \zeta_{1_{\boldsymbol{\nu}_{p-1}}}^{b} \partial_{\boldsymbol{\alpha}_{k} \beta_{p}}^{k+1} \zeta_{\boldsymbol{\beta}_{p-1}}^{c} \partial U_{\left.\mid \boldsymbol{\mu}_{p}\right] b}^{a \boldsymbol{\nu}_{p-1}} / \partial \partial_{\boldsymbol{\alpha}_{k}}^{k} \phi_{\boldsymbol{\beta}_{p}}^{c}\right. \\
& \left.+\sum_{0 \leq k \leq 1} \partial_{\alpha} \zeta_{1_{\boldsymbol{\nu}_{p-1}}^{b}}^{b} \partial_{\boldsymbol{\alpha}_{k} \beta_{p}}^{k+1} \zeta_{\boldsymbol{\beta}_{p-1}}^{c} \partial \stackrel{V}{V}_{\left.\mid \boldsymbol{\mu}_{p}\right] b}^{a \boldsymbol{\nu}_{p-1} \alpha} / \partial \partial_{\boldsymbol{\alpha}_{k}}^{k} \phi_{\boldsymbol{\beta}_{p}}^{c}\right)-\left(\zeta_{1} \leftrightarrow \zeta_{2}\right) .
\end{aligned}
$$

Substitution into this equation using an explicit general linear form for $\stackrel{(1)}{V}$ and $\stackrel{(1)}{U}$ in terms of $\phi$ and derivatives of $\phi$ yields determining equations on their coefficients. These equations are readily solved by using the jet-space (algebraic) tensor techniques shown in Ref. 14].

The solution for $\stackrel{(1)}{V}$ and $\stackrel{(1)}{U}$ determines the lowest order part of the infinitesimal gauge group structure $\left[\delta_{\zeta_{1}}{ }^{(0)} \delta_{\zeta_{2}}\right]=\delta_{\zeta_{3}}^{(0)}$ where the parameter $\zeta_{3}$ is given in terms of $\zeta_{1}$ and $\zeta_{2}$ to 
lowest order by the closure relation

$$
\left.\delta_{\zeta_{1}}^{(0)} \delta_{\zeta_{2}}^{(1)} \phi_{\boldsymbol{\mu}_{p}}^{a}-\left(\zeta_{1} \leftrightarrow \zeta_{2}\right)=\partial_{\left[\mu_{p}\right.} \stackrel{(0)}{\zeta}_{3}^{a} \boldsymbol{\mu}_{p-1}\right]
$$

which follows from equation (2.53).

Closure of the gauge group at 1st order is derived as the next step from the following analysis of the determining equations at 1st order in the hierarchy. Consider the Lie derivative commutator equation (2.44) minus the Lie derivative equation (2.43) applied to the gauge group commutator parameter $\zeta=\stackrel{(0)}{\zeta_{3}}$,

$$
0=\mathcal{L}_{\delta_{\left[\zeta_{1}, \zeta_{2}\right]} \phi} \stackrel{(1)}{\boldsymbol{\mu}_{a}} \underset{a}{\boldsymbol{\mu}_{p}}=\stackrel{(1)}{\delta_{\left[\zeta_{1}, \zeta_{2}\right]}} \stackrel{\stackrel{(1)}{E}}{a} \boldsymbol{\mu}_{p}+\stackrel{(1)}{E} \text { terms }
$$

where

$$
\delta_{\left[\zeta_{1}, \zeta_{2}\right]} \phi_{\boldsymbol{\mu}_{p}}^{a}=\left[\delta_{\zeta_{1}}, \delta_{\zeta_{2}}\right] \phi_{\boldsymbol{\mu}_{p}}^{a}-\delta_{\zeta_{3}} \phi_{\boldsymbol{\mu}_{p}}^{a}
$$

denotes the field variation representing the deviation from closure of the deformed gauge symmetry group. When $\phi$ is taken to satisfy the linear field equation, the $\stackrel{(1)}{E}$ terms drop out of equation (2.56), yielding

$$
0=\left.\left(\stackrel{(1)}{\delta_{\left[\zeta_{1}, \zeta_{2}\right]}}{\stackrel{(1)}{\boldsymbol{\mu}_{p}}}_{a}\right)\right|_{E=0}
$$

Lemma 2.6 implies

$$
\stackrel{(1)}{\delta}_{\left[\zeta_{1}, \zeta_{2}\right]} \phi_{\boldsymbol{\mu}_{p}}^{a}=\partial_{\left[\mu_{p}\right.}{\left.\stackrel{(1)}{\zeta}{ }^{a} \boldsymbol{\mu}_{p-1}\right]}^{(1)}
$$

for some locally constructed parameter $\zeta_{3}$, which determines the deformation of the gauge group structure at 1 st order. Hence a closure result is obtained.

Theorem 2.7: The deformed gauge symmetry generates a gauge group that closes up to 1 st order on the solution space deformed field equation

$$
\left.\delta_{\left[\zeta_{1}, \zeta_{2}\right]} \phi\right|_{E=0}=\stackrel{(0)}{\delta}_{\left[\zeta_{1}, \zeta_{2}\right]} \phi+\left.\stackrel{(1)}{\delta}_{\left[\zeta_{1}, \zeta_{2}\right]} \phi\right|_{E=0} ^{(1)}+\cdots=0 \text { up to } 1 \text { st order }
$$

Closure can be shown to extend in a similar way to higher orders, leading to an infinitesimal gauge group structure

$$
\left.\left[\delta_{\zeta_{1}}, \delta_{\zeta_{2}}\right]\right|_{E=0}=\left.\delta_{\zeta_{3}}\right|_{E=0}
$$

with

$$
\zeta_{3}=\stackrel{(0)}{\zeta_{3}}+\stackrel{(1)}{\zeta_{3}}+\cdots
$$

depending bilinearly on $\zeta_{1}, \zeta_{2}$ (and their derivatives). 
This theorem gives rise to an obstruction for existence of quadratic terms in the deformed gauge symmetry. Take the parameter to be rigid, $\left.\zeta\right|_{\text {rigid }}=$ const., so then the curl of the gauge group closure equation (2.60) restricted to rigid symmetries yields

$$
\begin{aligned}
& 0=\left.\partial_{[\nu}\left(\left[\stackrel{(1)}{\delta}_{\left.\zeta_{1}\right|_{\text {rigid }}},\left.\stackrel{(1)}{\delta} \zeta_{2}\right|_{\text {rigid }}\right] \phi_{\left.\boldsymbol{\mu}_{p}\right]}^{a}-\stackrel{(1)}{\delta}_{\left.\zeta_{3}\right|_{\text {rigid }}}^{\phi} \phi_{\left.\boldsymbol{\mu}_{p}\right]}^{a}\right)\right|_{E=0} ^{(1)} \\
& =\partial_{[\nu \mid}\left(\left.\left.\zeta_{2}^{b}{ }_{\boldsymbol{\nu}_{p-1}}^{b}\right|_{\text {rigid }} \zeta_{1}^{d}{ }_{\boldsymbol{\rho}_{p-1}}^{d}\right|_{\text {rigid }} \sum_{0 \leq k \leq 2}\left(\partial_{\boldsymbol{\alpha}_{k}}^{k} \stackrel{(1)}{U_{\boldsymbol{\beta}_{p} d}^{c \boldsymbol{\rho}_{p-1}}}\right) \partial \stackrel{(1)}{U}_{\left.\mid \boldsymbol{\mu}_{p}\right] b}^{a \boldsymbol{\nu}_{p-1}} / \partial \partial_{\boldsymbol{\alpha}_{k}}^{k} \phi_{\boldsymbol{\beta}_{p}}^{c}-\left(\zeta_{1} \leftrightarrow \zeta_{2}\right)\right. \\
& \left.-\stackrel{(0)}{\zeta}_{3}^{b} \boldsymbol{\nu}_{p-1} \mid \stackrel{(1)}{\text { rigid }} U_{\left.\mid \boldsymbol{\mu}_{p}\right] b}^{a \boldsymbol{\nu}_{p-1}}\right)\left.\right|_{(1)=0} .
\end{aligned}
$$

This equation is a rigidity-type integrability condition on the linear deformation terms, namely, further necessary determining equations on the coefficients of $\phi$ and derivatives of $\phi$ in $\stackrel{(1)}{U}$.

An additional rigidity-type integrability condition on the linear deformation terms arises from the 1st order Lie derivative equation (2.43) with $\zeta$ taken to be constant, $0=\mathcal{L}_{\delta_{\left.\zeta\right|_{\text {rigid }}} \phi} \stackrel{\stackrel{(1)}{E}}{\boldsymbol{\mu}_{p}}$. Factorization of the parameter $\left.\zeta_{\boldsymbol{\alpha}_{p-1}}^{c}\right|_{\text {rigid }}$ out of this equation gives

$$
\left.0=\sum_{0 \leq k \leq 2}\left(\partial_{\boldsymbol{\alpha}_{k}}^{k} \stackrel{(1)}{U}_{\boldsymbol{\nu}_{p} c}^{\boldsymbol{\rho}_{p-1}}\right) \partial \stackrel{(1)}{\boldsymbol{\mu}_{p}} / \partial \partial_{\boldsymbol{\alpha}_{k}}^{k} \phi_{\boldsymbol{\nu}_{p}}^{b}+(-1)^{k} \partial_{\boldsymbol{\alpha}_{k}}^{k} \stackrel{(1)}{E_{b}^{\boldsymbol{\nu}_{p}}} \partial U_{\boldsymbol{\nu}_{p} c}^{(1)} b \boldsymbol{\alpha}_{p-1} / \partial \partial_{\boldsymbol{\alpha}_{k}}^{k} \phi_{\boldsymbol{\mu}_{p}}^{a}\right) .
$$

This imposes more determining equations on the coefficients of $\phi$ and derivatives of $\phi$ in $\stackrel{(1)}{U}$.

The preceding equations are readily solved by the same algebraic techniques as discussed for the commutator equation (2.54). There is a useful remark to make here. If a classification of locally constructed linear symmetries depending on a rigid parameter $\left.\zeta\right|_{\text {rigid }}$ is already known for the linear field equation, these symmetries then comprise all possible linear deformation terms for $\stackrel{(1)}{U}$, since existence of a rigid symmetry $\left.\stackrel{(1)}{\delta}\right|_{\zeta \text { rigid }} \phi_{\boldsymbol{\mu}_{p}}^{a}=\left.U_{\boldsymbol{\mu}_{p} b}^{a \nu_{p-1}} \zeta_{\boldsymbol{\nu}_{p-1}}^{b}\right|_{\text {rigid }}$ is necessary to satisfy the integrability conditions (2.63) and (2.64). Moreover, the Noether currents associated with these symmetries comprise the corresponding possible quadratic deformation terms for $\stackrel{(2)}{E}$, since the conservation law for a Noether current is the same as the gauge invariance identity $\partial_{\mu}\left(\left.\stackrel{(2)}{E}_{b}^{\mu \boldsymbol{\nu}_{p-1}} \zeta_{\boldsymbol{\nu}_{p-1}}^{b}\right|_{\text {rigid }}\right)=\left.\stackrel{(1)}{E}_{a}^{\boldsymbol{\alpha}_{p}} \stackrel{(1)}{U}_{\boldsymbol{\alpha}_{p} b}^{a \boldsymbol{\nu}_{p-1}} \zeta_{\boldsymbol{\nu}_{p-1}}^{b}\right|_{\text {rigid }}$ from proposition 2.5, and hence $\left.\stackrel{(2)}{E}_{b}^{\mu \boldsymbol{\nu}_{p-1}} \zeta_{\boldsymbol{\nu}_{p-1}}^{b}\right|_{\text {rigid }}$ necessarily agrees with the Noether current to within an identically divergence-free term. Thus, any symmetry of a rigid form that may be available in the linear abelian theory can be used as a starting point for solving the deformation determining equations. This observation is often referred to as the "method of gauging a rigid symmetry" 
or the "Noether coupling method" and it has been used extensively in supergravity contexts 2, 40]. Of course, it is unable to provide a complete classification or a uniqueness result for deformations.

Once the linear deformation terms have been determined, the next steps are to solve for the quadratic deformation terms in the gauge symmetry and in the field equation, respectively, from the 1st order closure equation (2.59)

$$
\left.0=\partial_{[\nu}\left(\stackrel{(0)}{\delta_{\zeta_{1}}^{(2)}} \delta_{\zeta_{2}}^{(2)}\right] \phi_{\left.\boldsymbol{\mu}_{p}\right]}^{a}-\left(\zeta_{1} \leftrightarrow \zeta_{2}\right)+\left[\stackrel{(1)}{\delta_{\zeta_{1}}}, \hat{\delta}_{\zeta_{2}}^{(1)}\right] \phi_{\left.\boldsymbol{\mu}_{p}\right]}^{a}-{\stackrel{\delta_{(0)}^{(1)}}{\zeta_{3}}}_{\left.\boldsymbol{\mu}_{p}\right]}^{a}\right)\left.\right|_{E=0} ^{(1)}
$$

and from the 1st order Lie derivative equation (2.43)

$$
0={\stackrel{(0)(2)}{\delta} \stackrel{\mu}{E}_{a}^{\boldsymbol{\mu}_{p}}}_{a} \underset{\mathcal{L}_{(1)} \stackrel{(1)}{E_{\zeta}}}{\boldsymbol{\mu}_{p}},
$$

with the gauge parameters no longer being rigid. Through the closure equation (2.55) at lowest order, the commutativity-type integrability condition on equation (2.66) reduces to

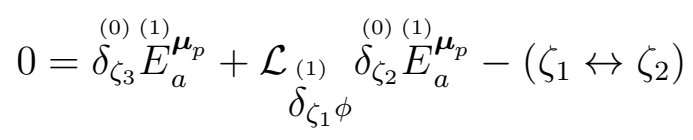

which is satisfied due to abelian gauge invariance. Then, an analysis of the commutativitytype integrability condition on equation (2.65) shows it is satisfied due to the lowest order part of the Jacobi identity applied to the gauge symmetries. Hence no obstructions arise for solving the 1st order Lie derivative equations (2.66) and (2.65).

To continue, after the expressions determined for $\stackrel{(1)}{U}$ and $\stackrel{(1)}{V}$ from prior equations are substituted, the determining equations (2.66) and (2.65) expand out to have the form

$$
\begin{aligned}
& \partial_{[\nu \mid}\left(\sum_{0 \leq k \leq 2} \zeta_{2}^{b} \partial_{\boldsymbol{\nu}_{p-1}}^{b+1} \partial_{\boldsymbol{\alpha}_{k} \beta_{p}}^{k+1} \zeta_{\boldsymbol{\beta}_{p-1}}^{c} \partial U_{\left.\mid \boldsymbol{\mu}_{p}\right] b}^{a \boldsymbol{\nu}_{p-1}} / \partial \partial_{\boldsymbol{\alpha}_{k}}^{k} \phi_{\boldsymbol{\beta}_{p}}^{c}\right. \\
& \left.+\sum_{0 \leq k \leq 1} \partial_{\alpha} \zeta_{2}^{b}{ }_{\boldsymbol{\nu}_{p-1}}^{b} \partial_{\boldsymbol{\alpha}_{k} \beta_{p}}^{k+1} \zeta_{\boldsymbol{\beta}_{p-1}}^{c} \partial V_{\left.\mid \boldsymbol{\mu}_{p}\right] b}^{a \boldsymbol{\nu}_{p-1} \alpha} / \partial \partial_{\boldsymbol{\alpha}_{k}}^{k} \phi_{\boldsymbol{\beta}_{p}}^{c}\right)-\left(\zeta_{1} \leftrightarrow \zeta_{2}\right) \\
& =\partial_{[\nu \mid}\left(\left(\sum_{0 \leq k \leq 2} \zeta_{1_{\boldsymbol{\nu}_{p-1}}^{b}}^{b} \partial_{\boldsymbol{\alpha}_{k}}^{k} \stackrel{(1)}{U}_{\boldsymbol{\beta}_{p} d}^{c \boldsymbol{\gamma}_{p-1}} \zeta_{2}^{d} \boldsymbol{\gamma}_{p-1}+\stackrel{(1)}{V}{ }_{\boldsymbol{\beta}_{p} d}^{c \boldsymbol{\gamma}_{p-1} \beta} \partial_{\beta} \zeta_{2}^{d} \boldsymbol{\gamma}_{p-1}\right) \partial U_{\left.\mid \boldsymbol{\mu}_{p}\right] b}^{a \boldsymbol{\nu}_{p-1}} / \partial \partial_{\boldsymbol{\alpha}_{k}}^{k} \phi_{\boldsymbol{\beta}_{p}}^{c}\right. \\
& \left.+\sum_{0 \leq k \leq 1} \partial_{\alpha} \zeta_{1_{\boldsymbol{\nu}_{p-1}}}^{b} \partial_{\boldsymbol{\alpha}_{k}}^{k}\left(\stackrel{(1)}{U}_{\boldsymbol{\beta}_{p} d}^{c \boldsymbol{\gamma}_{p-1}} \zeta_{2}^{d} \boldsymbol{\gamma}_{p-1}+\stackrel{(1)}{V}_{\boldsymbol{\beta}_{p} d}^{c \boldsymbol{\gamma}_{p-1} \beta} \partial_{\beta} \zeta_{2}^{d}{\boldsymbol{\boldsymbol { \gamma } _ { p - 1 }}}^{d}\right) \partial \stackrel{(1)}{V}_{\left.\mid \boldsymbol{\mu}_{p}\right] b}^{a \boldsymbol{\nu}_{p-1} \alpha} / \partial \partial_{\boldsymbol{\alpha}_{k}}^{k} \phi_{\boldsymbol{\beta}_{p}}^{c}\right)
\end{aligned}
$$

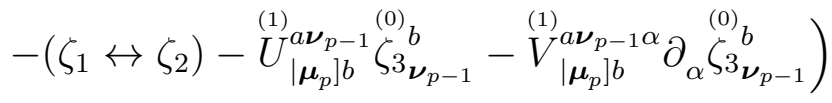

and

$$
\sum_{0 \leq k \leq 2} \partial_{\boldsymbol{\alpha}_{k} \beta_{p}}^{k+1} \zeta_{\boldsymbol{\beta}_{p-1}}^{c} \partial \stackrel{(2)}{E}_{a}^{\boldsymbol{\mu}_{p}} / \partial \partial_{\boldsymbol{\alpha}_{k}}^{k} \phi_{\boldsymbol{\beta}_{p}}^{c}
$$




$$
\begin{aligned}
= & -\sum_{0 \leq k \leq 2} \partial_{\boldsymbol{\alpha}_{k}}^{k} \stackrel{(1)}{\left.U_{\boldsymbol{\beta}_{p} d}^{c \boldsymbol{\gamma}_{p-1}} \zeta_{\gamma_{p-1}}^{d}+\stackrel{(1)}{V_{\boldsymbol{\beta}_{p} d}^{c \boldsymbol{\gamma}_{p-1} \beta}} \partial_{\beta} \zeta_{\gamma_{p-1}}^{d}\right) \partial{ }^{(1)} \boldsymbol{\mu}_{a}} / \partial \partial_{\boldsymbol{\alpha}_{k}}^{k} \phi_{\boldsymbol{\beta}_{p}}^{c} \\
& -\sum_{0 \leq k \leq 1}(-1)^{k} \partial_{\boldsymbol{\alpha}_{k}}^{k}\left(\partial_{\beta} \zeta_{\boldsymbol{\beta}_{p-1}}^{c} \stackrel{(1)}{E_{b}^{\boldsymbol{\nu}_{p}}} \partial \stackrel{(1)}{V}_{\boldsymbol{\nu}_{p} c}^{b \boldsymbol{\beta}_{p-1} \beta} / \partial \partial_{\boldsymbol{\alpha}_{k}}^{k} \phi_{\boldsymbol{\mu}_{p}}^{a}\right) \\
& -\sum_{0 \leq k \leq 2}(-1)^{k} \partial_{\boldsymbol{\alpha}_{k}}^{k}\left(\zeta_{\boldsymbol{\beta}_{p-1}}^{c} \stackrel{(1)}{E_{b}^{\boldsymbol{\nu}_{p}}} \partial \stackrel{(1)}{U}_{\boldsymbol{\nu}_{p} c}^{b \boldsymbol{\beta}_{p-1}} / \partial \partial_{\boldsymbol{\alpha}_{k}}^{k} \phi_{\boldsymbol{\mu}_{p}}^{a}\right)
\end{aligned}
$$

where

$$
\stackrel{(2)}{E_{a}^{\boldsymbol{\mu}_{p}}}=\stackrel{(1)}{\boldsymbol{\mu}_{p} \boldsymbol{\mu}_{p} \alpha \beta} \partial_{\alpha} \partial_{\beta} \phi_{\boldsymbol{\nu}_{p}}^{b}+\stackrel{(1)}{P} \underset{a b}{\boldsymbol{\mu}_{p} \boldsymbol{\nu}_{p} \alpha} \partial_{\alpha} \phi_{\boldsymbol{\nu}_{p}}^{b}+\stackrel{(1)}{\boldsymbol{\mu}_{a b} \boldsymbol{\mu}_{p}} \phi_{\boldsymbol{\nu}_{p}}^{b} .
$$

Despite their complicated appearance, it is computationally straightforward to solve these equations for $\stackrel{(2)}{U}$ and $\stackrel{(2)}{V}$ by use of the jet-space (algebraic) tensor techniques in Ref. [14].

\section{E. Geometrical structure of lowest-order deformations}

Strikingly, a simple explicit solution for the quadratic deformation terms can be constructed out of the linear deformations terms $\stackrel{(1)}{U}$ and $\stackrel{(1)}{V}$ under a mild restriction on the form sought for the deformed field equation and gauge symmetry. The result relies on the solution of the 0th order Lie derivative commutator equation.

Theorem 2.8: Restrict deformations so the field equation and the gauge symmetry contain no more derivatives of $\phi$ and $\zeta$, in total, than the number in the linear field equation (2.4) and the abelian gauge symmetry (2.1) (namely, in notation (2.26) and (2.30), $Q, R$, and $V$ have no dependence on derivatives of $\phi$, while $P$ and $U$ have at most linear dependence on derivatives of $\phi$, with higher order derivatives excluded). Then, up to a nonlinear change of field variable and of gauge parameter, the linear deformation terms have the form

$$
\stackrel{(1)}{V_{\boldsymbol{\mu}_{p} b}^{a \boldsymbol{\nu}_{p-1} \alpha}}=0, \quad \stackrel{(1)}{\boldsymbol{\mu}_{\boldsymbol{\mu}_{p} b}^{a \nu_{p-1}}}=u_{0 b c}^{\boldsymbol{\nu}_{p-1} a} \phi_{\boldsymbol{\mu}_{p}}^{c}+u_{1}^{\boldsymbol{\nu}_{p} b c}{ }_{\boldsymbol{\mu}_{p-1} \boldsymbol{\alpha}_{p+1} a}^{\boldsymbol{\nu}_{p}} \Phi_{\boldsymbol{\alpha}_{p+1}}^{c}
$$

for some coefficients such that $u_{0}$ is constant, skew in its lower internal indices for $p=1$, and vanishes for $p>1$; the quadratic deformation terms are explicitly given by

$$
\begin{aligned}
& \stackrel{(2)}{V}_{\boldsymbol{\mu}_{p} b}^{a \boldsymbol{\nu}_{p-1} \alpha}=0,
\end{aligned}
$$

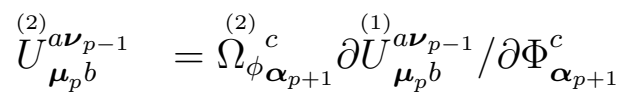

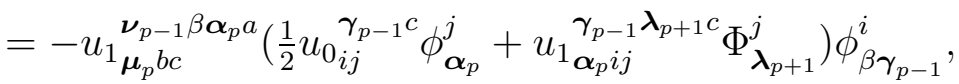

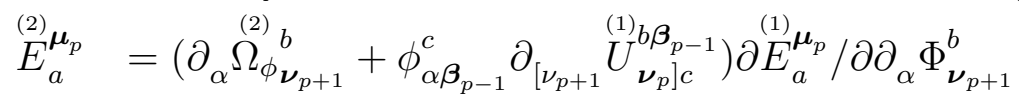




$$
\begin{aligned}
& +\stackrel{(2)}{\Omega}_{\phi_{\boldsymbol{\nu}_{p+1}}} \partial{\stackrel{(1)}{E_{a}}}_{a}^{\boldsymbol{\mu}_{p}} / \partial \Phi_{\boldsymbol{\nu}_{p+1}}^{b}+\stackrel{(1)}{E}_{b}^{\boldsymbol{\nu}_{p}} \phi_{\alpha \boldsymbol{\beta}_{p-1}}^{c} \partial U_{\boldsymbol{\nu}_{p} c}^{b \boldsymbol{\beta}_{p-1}} / \partial \Phi_{\alpha \boldsymbol{\mu}_{p}}^{a} \\
& =-q_{a b}^{\boldsymbol{\mu}_{p} \boldsymbol{\nu}_{p+1} \alpha}\left(\partial_{\alpha}\left(\left(\frac{1}{2} u_{0_{i j}}^{\boldsymbol{\gamma}_{p-1} b} \phi_{\boldsymbol{\nu}_{p}}^{j}+u_{\boldsymbol{\nu}_{p} i j}^{\boldsymbol{\gamma}_{p-1} \boldsymbol{\lambda}_{p+1} b} \Phi_{\boldsymbol{\lambda}_{p+1}}^{j}\right) \phi_{\nu_{p+1} \boldsymbol{\gamma}_{p-1}}^{i}\right)\right. \\
& \left.+\phi_{\alpha \boldsymbol{\beta}_{p-1}}^{c}\left(u_{0}^{\boldsymbol{\beta}_{c e-1} b} \Phi_{\boldsymbol{\nu}_{p+1}}^{e}+\partial_{\left[\nu_{p+1}\right.}\left(u_{\left.\boldsymbol{\nu}_{p}\right] c e}^{\boldsymbol{\beta}_{p-1} \boldsymbol{\gamma}_{p+1} b} \Phi_{\boldsymbol{\gamma}_{p+1}}^{e}\right)\right)\right) \\
& -p_{a b}^{\boldsymbol{\mu}_{p} \boldsymbol{\nu}_{p+1}}\left(\frac{1}{2} u_{0_{i j}}^{\boldsymbol{\gamma}_{p-1} b} \phi_{\boldsymbol{\nu}_{p}}^{j}+u_{\boldsymbol{\nu}_{p} i j}^{\boldsymbol{\gamma}_{p-1} \boldsymbol{\lambda}_{p+1} b} \Phi_{\boldsymbol{\lambda}_{p+1}}^{j}\right) \phi_{\nu_{p+1} \boldsymbol{\gamma}_{p-1}}^{i} \\
& +u_{\boldsymbol{\nu}_{p} e a}^{\boldsymbol{\gamma}_{p-1} \beta \boldsymbol{\mu}_{p} b}\left(q_{b c}^{\boldsymbol{\nu}_{p} \boldsymbol{\lambda}_{p+1} \alpha} \partial_{\alpha} \Phi_{\boldsymbol{\lambda}_{p+1}}^{c}+p_{b c}^{\boldsymbol{\nu}_{p} \boldsymbol{\lambda}_{p+1}} \Phi_{\boldsymbol{\lambda}_{p+1}}^{c}\right) \phi_{\beta \boldsymbol{\gamma}_{p-1}}^{e} \\
& =\stackrel{(1)}{Q}{ }_{a b}^{\boldsymbol{\mu}_{p} \boldsymbol{\nu}_{p} \alpha \beta} \partial_{\alpha} \partial_{\beta} \phi_{\boldsymbol{\nu}_{p}}^{b}+\stackrel{(1)}{P_{a b}^{\boldsymbol{\mu}_{p} \boldsymbol{\nu}_{p} \alpha}} \partial_{\alpha} \phi_{\boldsymbol{\nu}_{p}}^{b}+\stackrel{(1)}{R}_{a b}^{\boldsymbol{\mu}_{p} \boldsymbol{\nu}_{p}} \phi_{\boldsymbol{\nu}_{p}}^{b},
\end{aligned}
$$

where

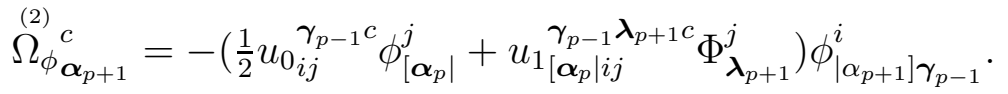

Note the coefficients $u_{0}$ and $u_{1}$ are also subject to additional algebraic equations that come from the integrability conditions (2.63) and (2.64).

Remark: As a result of proposition 2.4, the restriction assumed on derivatives is necessary if deformations to all orders are required both to preserve the number of dynamical degrees of freedom of $\phi$ from the linear theory and to contain a bounded total number of derivatives of $\phi$.

In outline, the proof of the theorem rests on using the determining equation (2.54) to show, firstly, that the solution for $\stackrel{(1)}{V}$ vanishes after a suitable change of field variable (2.39) and gauge parameter (2.40) are made; secondly, the solution for $\stackrel{(1)}{U}$ establishes that the curl of this expression is gauge invariant with respect to the $\stackrel{(0)}{\delta}_{\zeta} \phi$ and that the exterior product of $\stackrel{(1)}{U}$ and $\stackrel{(0)}{\delta_{\zeta}} \phi$ is an exact variation,

$$
\stackrel{(0)}{\delta_{\zeta}} \partial_{[\alpha} \stackrel{(1)}{U_{\left.\boldsymbol{\mu}_{p}\right] b}^{a \nu_{p-1}}}=0, \quad \stackrel{(1)}{U_{\left[\boldsymbol{\mu}_{p-1} \mid b\right.}^{a \boldsymbol{\mu}_{p}}} \partial_{\mid \alpha]} \zeta_{\boldsymbol{\nu}_{p-1}}^{b}=-\stackrel{(0)}{\delta_{\zeta}} \stackrel{(2)}{\Omega}_{\phi_{\boldsymbol{\mu}_{p} \alpha} \alpha}^{a}
$$

This analysis is a straightforward application of the jet-space tensor techniques from Ref. [14]. Then, from expressions (2.77), the Lie derivative equation (2.69) is easily solved for $\stackrel{(2)}{E}$, as is the closure equation (2.68) for $\stackrel{(2)}{\delta_{\zeta}} \phi$ in terms of the commutator of $\stackrel{(1)}{\delta_{\zeta}} \phi$. Note the expression obtained for $\stackrel{(2)}{E}$ is essentially a construction of the Noether current of the rigid symmetry $\stackrel{(1)}{\delta}_{\text {rigid }} \phi=\stackrel{(1)}{U}$, due to the Noether gauge-invariance identity stated in proposition 2.5. Uniqueness of both $\stackrel{(2)}{E}$ and $\stackrel{(2)}{\delta_{\zeta}} \phi$ will be established later (see theorem 2.10).

These results (2.71) -(2.75) express the following geometrical structure arising from the linear and quadratic deformation terms: First observe, since $\stackrel{(1)}{V}=\stackrel{(2)}{V}=0$, the deformed 
gauge symmetry takes the form of a covariant derivative on the gauge parameter,

$$
\delta_{\zeta} \phi_{\boldsymbol{\mu}_{p}}^{a}=D_{\phi}(\zeta)_{\boldsymbol{\mu}_{p}}^{a}=\partial_{\left[\mu_{p}\right.} \zeta_{\left.\boldsymbol{\mu}_{p-1}\right]}^{a}+\left(u_{0 b c}^{\nu_{p-1} a} \phi_{\boldsymbol{\mu}_{p}}^{c}+u_{1}^{\boldsymbol{\mu}_{p-1} b c} \boldsymbol{\alpha}_{p+1} a \Phi_{\boldsymbol{\alpha}_{p+1}}^{c}\right) \zeta_{\boldsymbol{\nu}_{p-1}}^{b}+\cdots
$$

(to linear order) where $D_{\phi}$ is viewed as an exterior derivative operator from the gauge parameter vector bundle $\mathcal{P}$ into $\mathcal{S}$ (i.e. $D_{\phi}: \Lambda_{p-1}(M) \otimes \mathbb{V} \rightarrow \Lambda_{p}(M) \otimes \mathbb{V}$ ). This derivative operator has a natural extension to the vector bundle $\mathcal{S}$ (i.e. $D_{\phi}: \Lambda_{p}(M) \otimes \mathbb{V} \rightarrow \Lambda_{p+1}(M) \otimes$ $\mathbb{V})$, in particular acting on $\delta_{\zeta} \phi$ itself by

$$
\begin{aligned}
D_{\phi}\left(\delta_{\zeta} \phi\right)_{\boldsymbol{\mu}_{p+1}}^{a}= & \partial_{\left[\mu_{p+1}\right.} \delta_{\zeta} \phi_{\left.\boldsymbol{\mu}_{p}\right]}^{a}+\left(u_{0 b c}^{\boldsymbol{\nu}_{p-1} a} \phi_{\left[\boldsymbol{\mu}_{p} \mid\right.}^{c}+u_{1\left[\boldsymbol{\mu}_{p} \mid b c\right.}^{\boldsymbol{\nu}_{p-1} \boldsymbol{\alpha}_{p+1} a} \Phi_{\boldsymbol{\alpha}_{p+1}}^{c}\right) \delta_{\zeta} \phi_{\left.\mid \mu_{p+1}\right] \boldsymbol{\nu}_{p-1}}^{b}+\cdots \\
= & \partial_{\left[\mu_{p+1} \mid\right.}\left(u_{0 b c}^{\boldsymbol{\nu}_{p-1} a} \phi_{\left.\mid \boldsymbol{\mu}_{p}\right]}^{c}+u_{\left.1 \mid \boldsymbol{\mu}_{p-1}\right] b c}^{\boldsymbol{\nu}_{p+1} a} \Phi_{\boldsymbol{\alpha}_{p+1}}^{c}\right) \zeta_{\boldsymbol{\nu}_{p-1}}^{b} \\
& \quad+2(1-1 / p)\left(u_{0 b c}^{\nu \boldsymbol{\nu}_{p-2} a} \phi_{\left[\boldsymbol{\mu}_{p} \mid\right.}^{c}+u_{1 \boldsymbol{\mu}_{p} \mid b c}^{\nu \boldsymbol{\nu}_{p-1} \boldsymbol{\alpha}_{p+1} a} \Phi_{\boldsymbol{\alpha}_{p+1}}^{c}\right)\left(\partial_{\left.\mid \mu_{p+1}\right]} \zeta_{\nu \boldsymbol{\nu}_{p-1}}^{b}\right. \\
& \left.+\partial_{\nu} \zeta_{\left.\mid \mu_{p+1}\right] \boldsymbol{\nu}_{p-2}}^{b}\right)+\cdots
\end{aligned}
$$

(to linear order). Next, the relation $(2.77)$ on $\stackrel{(1)}{U}$ gives rise to a nonlinear deformation of the abelian field strength $\Phi$ as defined by

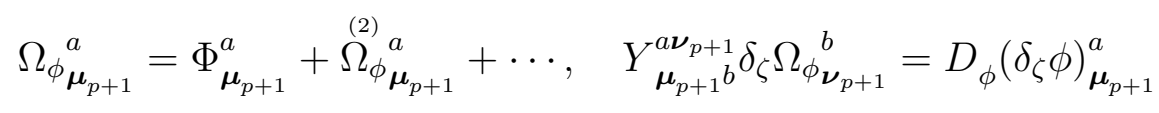

for some locally constructed linear map

$$
Y_{\boldsymbol{\mu}_{p+1} b}^{a \boldsymbol{\nu}_{p+1}}=\delta_{b}^{a} \delta_{\boldsymbol{\mu}_{p+1}}^{\boldsymbol{\nu}_{p+1}}+\stackrel{(1)}{Y}_{\boldsymbol{\mu}_{p+1} b}^{\boldsymbol{\nu}_{p+1}}+\cdots
$$

Notice this expands out to $\left.\partial_{\left[\mu_{p+1}\right.} \stackrel{(0)}{\delta}_{\zeta}^{a}\right|_{\left.\boldsymbol{\mu}_{p}\right]} ^{a}=\stackrel{(0)}{\delta}_{\zeta} \Phi_{\boldsymbol{\mu}_{p+1}}^{a}=0$ at lowest order, which holds as an identity; then at 1 st order, the cancellation of terms $\partial_{\left[\mu_{p+1}\right.} \hat{\delta}_{\zeta}^{(1)} \phi_{\left.\boldsymbol{\mu}_{p}\right]}^{a}=\stackrel{\delta}{\zeta}_{\delta^{(1)}}^{a} \boldsymbol{\mu}_{p+1}$ leaves $\stackrel{(1)}{D_{\phi}}\left(\stackrel{(0)}{\delta_{\zeta}} \phi\right)_{\boldsymbol{\mu}_{p+1}}^{a}=\stackrel{(0)}{\delta}_{\zeta}^{\left(\Omega_{\phi}\right.}{ }_{\boldsymbol{\mu}_{p+1}}$, which reduces to equation (2.77). The linear map $Y$ does not enter until next order. Thus, the nonlinear field strength $p+1$-form is given by

$$
\Omega_{\phi}^{a} \boldsymbol{\mu}_{p+1}=\partial_{\left[\mu_{p+1}\right.} \phi_{\left.\boldsymbol{\mu}_{p}\right]}^{a}+\left(u_{0 b c}^{\nu_{p-1} a} \phi_{\left[\boldsymbol{\mu}_{p} \mid\right.}^{c}+u_{\left[\boldsymbol{\mu}_{p} \mid b c\right.}^{\boldsymbol{\nu}_{p-1} \boldsymbol{\alpha}_{p+1} a} \Phi_{\boldsymbol{\alpha}_{p+1}}^{c}\right) \phi_{\left.\mid \mu_{p+1}\right] \boldsymbol{\nu}_{p-1}}^{b}+\cdots
$$

(to quadratic order). As a result, the geometrical content of deformations in theorem 2.8 is seen to be encoded in the covariant derivative operator $D_{\phi}$ and the nonlinear field strength $\Omega_{\phi}$ emerging from the solution for the linear terms $\stackrel{(1)}{U}, \stackrel{(1)}{V}$.

Corollary 2.9: In geometrical terms, the form of $\stackrel{(2)}{U}$ is given by a replacement of the abelian field strength $\Phi$ in $\stackrel{(1)}{U}$ by the quadratic part of the nonlinear field strength $\Omega_{\phi}$. 
Similarly, the form of $\stackrel{(2)}{E}$ is given by a simultaneous replacement of the quadratic field strength $\stackrel{(2)}{\Omega}_{\phi}$ for $\Phi$ and the linear part of the covariant derivative $\stackrel{(1)}{D_{\phi}}$ for the coordinate derivative operator, up to additional terms that vanish on the solution space $\stackrel{(1)}{E}=0$.

The scope of this theorem encompasses Yang-Mills and Freedman-Townsend theories, Einstein gravity theory and its multi-graviton generalizations, as well as the gravity-like generalization of Yang-Mills theory, in any dimension $d \geq 3$. Moreover, in $d=3$ dimensions, Yang-Mills Chern-Simons theory is included, as is combined Yang-Mills Freedman-Townsend theory, in addition to the torsion generalizations of Yang-Mills theory presented in Secs. [II] - V. The results in the theorem thus expose a hitherto unrecognized universal nonlinear structure present among a wide class of important examples of nonlinear gauge theories.

The only significant example excluded by the theorem is the exotic parity-violating multigraviton theories in $d=3,5$ dimensions, as they involve a more complicated type of deformation in which the form of the gauge symmetry is heavily coupled to a deformed auxiliary gauge freedom on the vielbein. In contrast, the auxiliary gauge freedom in ordinary gravitational theories is consistent with the form of the deformations given in the theorem. Basically, the expression for $\stackrel{(2)}{E}$ can be shown to preserve the number of degrees of gauge freedom from the linearized local Lorentz transformations present on the (linearized vielbein) field variable in $\stackrel{(1)}{E}$. A full discussion will be left to a forthcoming paper addressed to more general gravity-like nonlinear $p$-form theories for $p \geq 1$ in $d$ dimensions.

\section{F. Remarks on integrability}

The trivial nature of the commutativity-type integrability conditions up to 1st order in the hierarchy of determining equations extends to higher orders. However, the rigidity-type integrability conditions remain nontrivial at least up to next order, as follows. The Lie derivative equation at 2 nd order gives

$$
0=\underset{\delta_{\left.\zeta\right|_{\text {rigid }} \phi}}{\mathcal{L}_{(1)}} \stackrel{(2)}{\boldsymbol{\mu}_{p}}+\underset{\delta_{\left.\zeta\right|_{\text {rigid }} \phi}}{\mathcal{L}_{(2)}} \stackrel{(1)}{\boldsymbol{\mu}_{p}}
$$

which constitutes an integrability condition on the linear deformation terms, since the

quadratic deformation terms $\stackrel{(2)}{\delta_{\zeta}} \phi$ and $\stackrel{(2)}{E}$ are determined from $\stackrel{(1)}{\delta_{\zeta}} \phi$ by theorem 2.8. Use of jet-space tensor techniques then reduces the integrability condition to determining equa- 
tions on the coefficients of $\phi$ and derivatives of $\phi$ in $\stackrel{(1)}{U}$. These equations are nontrivial, as seen in examples.

In Freedman-Townsend theory and its Yang-Mills generalizations, the gauge groups close only on the solution space and their structure emerges purely from the integrability equation (2.83) at 2nd order. In contrast, for Yang-Mills theory and algebra-valued gravity theory, the nature of the closure and integrability equations is reversed - the gauge groups in those theories close off the solution space and their structure is determined entirely by the 1st order integrability condition coming from the closure equation (2.59), while the integrability equation (2.83) at 2nd order gives no extra structure or algebraic constraints. Finally, the exotic parity-violating multi-graviton theories share some features of all these other examples, with both the closure equation and integrability equation contributing to the structure of the gauge group.

\section{G. Uniqueness (rigidity) of deformations}

A strong uniqueness result for nonlinear deformation terms can be established by an induction argument in powers of the fields $\phi$ under the same restriction on derivatives in the form sought for deformations as assumed in theorem 2.8. To begin, consider two solutions of the determining equations to all orders, and let $\Delta \stackrel{(k)}{E}$ and $\Delta \stackrel{(k)}{\delta_{\zeta}} \phi$ denote the difference of the $k$ th power deformation terms in the solutions. Suppose these solutions agree for $k<n$ up to some power $n>1$, so $\Delta \stackrel{(k)}{E}=0$ and $\Delta \stackrel{(k)}{\delta_{\zeta}} \phi=0,0 \leq k \leq n-1$, with the lowest power terms automatically fixed by the form of the abelian gauge symmetry and linear field equation. Subtraction of the Lie derivative equations satisfied for each solution then yields

$$
0=\stackrel{(0)}{\delta_{\zeta}}(\Delta \stackrel{(n)}{E})
$$

to lowest nonvanishing order. From this equation it follows that $\Delta \stackrel{(n)}{E}$ is a gauge-invariant expression with respect to the abelian gauge symmetry and hence has the form of a homogeneous polynomial of degree $n>1$ in the abelian field strength $\Phi$. The restriction assumed on derivatives of $\phi$ requires $\Delta \stackrel{(n)}{E}$ to contain at most two derivatives in total, hence $\Delta \stackrel{(n)}{E}$ must vanish if $n>2$. In the case $n=2$, a simple analysis shows that the Frechet derivative of any homogeneous quadratic polynomial in $\Phi$ fails to be self-adjoint, which implies such polynomials cannot have the form of an Euler-Lagrange equation [35, 37]. Thus $\Delta \stackrel{(n)}{E}$ must 
also vanish if $n=2$.

Now, by subtraction of the Lie derivative commutator equations for each solution, $\Delta{\stackrel{(n)}{\delta_{\zeta}} \phi}^{\prime}$ satisfies

$$
0=\delta_{\zeta_{2}}^{(0)}\left(\left.\Delta \delta_{\zeta_{1}}^{(n)} \underset{E}{E}\right|_{(\underset{E}{(1)}=0}-\left(\zeta_{1} \leftrightarrow \zeta_{2}\right)\right.
$$

to lowest nonvanishing order. An analysis of this equation using lemma 2.6 shows that the

curl of $\delta_{\zeta_{2}}^{(0)}\left(\Delta \delta_{\zeta_{1}}^{(n)} \phi\right)$ is zero, after all change of variable freedom (2.39) and (2.40) in $\phi$ and $\zeta$ is exhausted. Hence $\Delta \stackrel{(n)}{\delta_{\zeta}} \phi$ must have the form of a homogeneous polynomial of degree $n>1$ in the abelian field strength $\Phi$. But, with the restriction assumed on derivatives of $\phi, \Delta \delta_{\zeta}^{(n)} \phi$ is required to contain at most one derivative in total, and thus it must vanish if $n \geq 2$.

Induction on the power $n$ therefore establishes the following uniqueness (rigidity) result.

Theorem 2.10: Up to a nonlinear change of field variable and of gauge parameter, deformations that contain, in total, no more derivatives of $\phi$ and $\zeta$ than the number in the linear abelian theory are uniquely determined by their lowest-order terms ${ }_{\zeta}^{(1)} \phi$.

\section{YANG-MILLS CHERN-SIMONS GAUGE THEORY WITH FREEDMAN- TOWNSEND INTERACTION}

The starting point here will be the linear abelian theory of a 1-form potential $A_{\mu}^{a}$ with an associated internal vector space structure $\mathbb{V}$ of dimension $n \geq 1$, on a $d=3$ dimensional spacetime. For simplicity, the spacetime is taken to be flat, with a metric $g_{\mu \nu}$ and a volume form $\epsilon_{\mu \nu \alpha}$ given in Minkowski coordinates. The standard Lagrangian for this theory is expressed in terms of the abelian field strength 2-form $F_{\mu \nu}^{a}=\partial_{[\mu} A_{\nu]}^{a}$ by

$$
\stackrel{(2)}{L}=\frac{1}{2} g^{\mu \nu} k_{a b} * F_{\mu}^{a} * F_{\nu}^{b}
$$

where $* F_{\mu}^{a}=\epsilon_{\mu}^{\alpha \beta} F_{\alpha \beta}^{a}$ is the dual field strength 1-form. The Lagrangian is gauge invariant under the abelian gauge symmetry

$$
{\stackrel{(0)}{\delta_{\zeta}}}^{a}{ }_{\mu}=\partial_{\mu} \zeta^{a}
$$

for an arbitrary function $\zeta^{a}$ on $M$ with values in $\mathbb{V}$. The linear field equation obtained via the Euler operator applied to $\stackrel{(2)}{L}$ is second order in derivatives and also is gauge invariant, as given by the curl of the dual field strength

$$
\stackrel{(1)}{E}_{a}^{\mu}=k_{a b} \epsilon^{\mu \alpha \beta} \partial_{\alpha} * F_{\beta}^{b} .
$$


Note this is the source-free Maxwell equation on $A_{\mu}^{a}$ in three dimensions. An abelian ChernSimons term is straightforwardly introduced through the Lagrangian

$$
\stackrel{(2)}{L}_{\mathrm{CS}}=\frac{1}{2} m g^{\mu \nu} k_{a b} A_{\mu}^{a} * F_{\nu}^{b}
$$

where $m$ is the Chern-Simons coupling constant. Under the abelian gauge symmetry $\stackrel{(0)}{\delta_{\zeta}} A_{\mu}^{a}$, the total Lagrangian $\stackrel{(2)}{L}+\stackrel{(2)}{L}_{\mathrm{CS}}$ remains invariant to within a divergence and thus contributes a gauge-invariant first derivative term

$$
\stackrel{(1)}{E}_{a}^{\mathrm{m} \mu}=m k_{a b} g^{\mu \nu} * F_{\nu}^{b}
$$

to the linear field equation $\stackrel{(1)}{E}_{a}^{\mu}+\stackrel{(1)}{E}_{a}^{\mathrm{m} \mu}=0$. Thus, the potential $A_{\mu}^{a}$ satisfies the field equation

$$
\epsilon_{\mu}^{\nu \alpha} \partial_{\nu} * F_{\alpha}^{a}=-m * F_{\mu}^{a}
$$

along with the identity

$$
\partial^{\mu} * F_{\mu}^{a}=0
$$

The physical content of solutions $A_{\mu}^{a}$ describes a set of free spin-one fields of mass $m$, as seen in a gauge invariant manner from the curl of the field equation (3.6),

$$
\square * F_{\mu}^{a}=m^{2} * F_{\mu}^{a} .
$$

Alternatively, in Lorentz gauge $\partial^{\mu} A_{\mu}^{a}=0$, when $m=0$ the field equation reduces to a set of massless linear vector wave equations

$$
\square A_{\mu}^{a}=0
$$

modulo the residual gauge freedom $A_{\mu}^{a} \rightarrow A_{\mu}^{a}+\partial_{\mu} \zeta^{a}$ such that $\square \zeta^{a}=0$. When $m \neq 0$, since both sides of the field equation (3.6) are a curl, this implies

$$
* F_{\mu}^{a}+m A_{\mu}^{a}=\partial_{\mu} \psi, \quad \square \psi=0,
$$

to within a gradient term $\psi$ that satisfies the scalar wave equation as a consequence of the Lorentz gauge on $A_{\mu}^{a}$ and the divergence free nature of $* F_{\mu}^{a}$. This $\psi$ term can be removed by the residual gauge freedom in $A_{\mu}^{a}$, so then

$$
* F_{\mu}^{a}=-m A_{\mu}^{a}
$$


and thus the field equation (3.6) becomes a set of massive linear vector wave equations

$$
\square A_{\mu}^{a}=m^{2} A_{\mu}^{a}
$$

It is interesting to note the relation (3.11) between the dual field strength and the potential in Lorentz gauge is equivalent to a pure Chern-Simons theory with an added mass term given by the Proca Lagrangian $\stackrel{(2)}{L}_{\mathrm{m}}=\frac{1}{2} m^{2} g^{\mu \nu} k_{a b} A_{\mu}^{a} A_{\nu}^{b}$. In both the massless and massive cases, two of the three independent components of each field $A_{\mu}^{a}$ are able to be gauged away, leaving a single dynamical degree of freedom for solutions of the field equation.

Nonlinear deformations of this linear abelian theory fall into two distinct classes, differing in how much of the spacetime structure they use. The first class consists of deformations that only rely on the spacetime metric and volume form along with exterior derivatives on the field and gauge parameter. Such deformations preserve the manifest Lorentz covariance of the linear abelian theory. The second class of deformations make essential use of Killing vectors of the spacetime metric and thus break Lorentz covariance. These non-covariant deformations will be considered in Sec. V.

Covariant deformations in the massless case were first studied systematically in Ref. [21], using the basic field-theoretic approach of Sec. III with deformation terms restricted to a quasilinear first-order derivative form for the gauge symmetry and a semilinear second-order derivative form for the field equation. These deformations to lowest order in powers of the fields comprise just the Yang-Mills type

$$
\stackrel{(1)}{\delta}_{\zeta} A_{\mu}^{a}=\kappa_{\mathrm{YM}} a_{b c}^{a} A_{\mu}^{b} \zeta^{c}, \quad \stackrel{(3)}{L}=\frac{1}{2} \kappa_{\mathrm{YM}} a_{a b c} \epsilon^{\mu \nu \alpha} * F_{\mu}^{a} A_{\nu}^{b} A_{\alpha}^{c},
$$

and the Freedman-Townsend type

$$
\stackrel{(1)}{\delta}_{\zeta} A_{\mu}^{a}=\kappa_{\mathrm{FT}} b_{b c}^{a} * F_{\mu}^{b} \zeta^{c}, \quad \stackrel{(3)}{L}=\frac{1}{2} \kappa_{\mathrm{FT}} b_{a b c} \epsilon^{\mu \nu \alpha} * F_{\mu}^{a} * F_{\nu}^{b} A_{\alpha}^{c}
$$

where the coefficients $a_{a b c}=k_{a d} a_{b c}^{d}$ and $b_{a b c}=k_{a d} b_{b c}^{d}$ are constant internal tensors on $\mathbb{V}$ satisfying the algebraic relations

$$
\begin{aligned}
& a_{a(b c)}=a_{(a b) c}=0, \quad a_{[b c}^{d} a_{a] d}^{e}=0, \\
& b_{a(b c)}=0
\end{aligned}
$$

An additional algebraic relation

$$
b_{e[a}^{d} b_{b c] d}=0
$$


arises from an integrability condition at next order. Here $\kappa_{\mathrm{YM}}, \kappa_{\mathrm{FT}}$ denote coupling constants.

Strong classification results have been obtained for lowest-order deformations of massless linear abelian $p$-form gauge theories in $d \geq 3$ dimensions [11, 12], relaxing the restrictions made on derivatives in the deformation terms in Ref. [21]. In particular, the results establish the uniqueness of these two types of covariant deformations in $d=3$ dimensions.

\section{A. Deformations with Chern-Simons term}

The deformation analysis is readily extended to the massive case, because the addition of a Chern-Simons term preserves the abelian gauge invariance. In outline, the 0th order closure equation on the deformed gauge symmetry is unchanged, and so the same linear deformation terms $\stackrel{(1)}{\delta}_{\zeta} A_{\mu}^{a}$ are obtained. As the key step, next the deformed gauge symmetry is found to remain compatible with the abelian Chern-Simons term in the linear field equation when the integrability condition from the 1st order determining equation on the deformed field equation is considered. Thus the same second derivative part for the quadratic deformation terms $\stackrel{(2)}{E}_{a}^{\mu}$ is obtained, while only the lower derivative part is changed due to the ChernSimons term. This yields

$$
\stackrel{(3)}{L}_{\mathrm{CS}}=\frac{1}{6} m \kappa_{\mathrm{YM}} a_{a b c} \epsilon^{\mu \nu \alpha} A_{\mu}^{a} A_{\nu}^{b} A_{\alpha}^{c} .
$$

As a result, lowest-order covariant deformations in the massive case comprise the same types as in the massless case, apart from the presence of the Chern-Simons term. However, an algebraic integrability condition occurs at the next order.

Additional algebraic integrability conditions emerge as well when the Yang-Mills and Freedman-Townsend types of deformations are combined.

Theorem 3.1: At lowest order, the most general allowed covariant deformation of the linear abelian gauge theory (3.1)-(3.7) restricted to a quasilinear first-order derivative form for $\stackrel{(1)}{\delta}_{\zeta} A_{\mu}^{a}$ and semilinear second-order derivative form for $\stackrel{(1)}{E}_{a}^{\mu}$ is a combination of the pure Yang-Mills and Freedman-Townsend types. The combined type involves an algebraic compatibility condition

$$
4 a_{e[c \mid[a} b_{b] \mid d]}^{e}+\kappa a_{c d}^{e} b_{a b e}-2 m \kappa b_{[b \mid c}^{e} b_{e \mid a] d}=0
$$


relating the constant coefficient tensors, where $\kappa=\kappa_{\mathrm{FT}} / \kappa_{\mathrm{YM}}$ is the ratio of coupling constants.

It is straightforward to complete these deformations to all higher orders, as carried out for the massless case in Ref. [22].

The algebraic structure (3.15) states that the tensor $a_{b c}^{a}$ represents the structure constants of a Lie algebra on $\mathbb{V}$ for which the symmetric tensor $k_{a b}$ is an invariant metric. In the case where $k_{a b}$ is positive definite, this is well known to imply $\left(\mathbb{V}, a^{a}{ }_{b c}\right)_{\mathrm{YM}}$ is a semisimple Lie algebra and $k_{a b}$ is its Cartan-Killing metric [41] (up to a scaling factor). In contrast, from the algebraic structure (3.16) and (3.17), the tensor $b^{a}{ }_{b c}$ is the co-adjoint representation of the structure constants $b_{c d}{ }^{e}=k^{e d} k_{d a} b^{a}{ }_{b c}$ of a Lie algebra on $\left(\mathbb{V}, k_{a b}\right)$, where the adjoint is defined with respect to the vector space metric $k_{a b}$. Note $k_{a b}$ is not required to be an invariant metric, so this Lie algebra $\left(\mathbb{V}, b_{b c}{ }^{a}\right)_{\mathrm{FT}}$ is allowed to be non-semisimple (for instance it can be solvable or nilpotent [41]).

For the combined Yang-Mills Freedman-Townsend type of covariant deformation, the algebraic relation (3.19) is satisfied by the identification of the two Lie algebras

$$
b_{b c}^{a}=a_{b c}^{a} \text { iff } \kappa=1 / m
$$

in which case $a_{b c}^{a}$ and $b_{b c}{ }^{a}$ are necessarily the structure constants of a semisimple Lie algebra on $\mathbb{V}$. Indeed, the lowest-dimensional example satisfying all the algebra structure for the combined deformation in the massive case is readily found to be $\kappa_{\mathrm{FT}}=m^{-1} \kappa_{\mathrm{YM}}$ and $\left(\mathbb{V}, a_{b c}^{a}\right)_{\mathrm{YM}}=\left(\mathbb{V}, b_{b c}{ }^{a}\right)_{\mathrm{FT}} \simeq S U(2)$, i.e. $\mathbb{V}$ is a three dimensional vector space on which the Lie algebra structure constants are given by $c^{a}{ }_{b c}=k^{a d} c_{b c d}$ where $c_{b c d}$ and $k_{a b}$ are a compatible volume form and positive-definite metric. However, this Lie algebra structure is incompatible for the massless case. In particular, for the example of a semisimple Lie algebra of lowest dimension, $\left(\mathbb{V}, a^{a}{ }_{b c}\right)_{\mathrm{YM}} \simeq S U(2)$, the compatibility relation (3.19) implies $\left(\mathbb{V}, b_{b c}{ }^{a}\right)_{\mathrm{FT}}$ is the Lie algebra of translations and a dilation in the Euclidean plane, as shown in Ref. [22]. This Lie algebra is degenerate, in contrast with $S U(2)$. More generally, a similar degeneracy occurs for $\left(\mathbb{V}, b_{b c}{ }^{a}\right)_{\mathrm{FT}}$ when higher dimensional semisimple Lie algebras are considered for $\left(\mathbb{V}, a_{b c}^{a}\right)_{\mathrm{YM}}$. A full investigation of the solutions of the algebraic compatibility relation (3.19) will be given elsewhere.

The algebraic obstruction in the massless case to combining Yang-Mills and Freedman- 
Townsend types of deformations with no Chern-Simons term reflects a basic difference in their geometrical structures. Consider a general semisimple Lie algebra $\left(\mathbb{V}, c_{b c}^{a}\right)=\mathfrak{g}$ in which the potential $A_{\mu}^{a}$ is assigned to take values, putting $a_{b c}^{a}=b_{b c}{ }^{a}=c^{a}{ }_{b c}$ for the YangMills and Freedman-Townsend Lie algebra structure tensors. In Yang-Mills theory, recall the gauge symmetry on $A_{\mu}^{a}$ geometrically is a covariant derivative

$$
\delta_{\zeta}^{\mathrm{YM}} A_{\mu}^{a}=\partial_{\mu} \zeta^{a}+\kappa_{\mathrm{YM}} a_{b c}^{a} A_{\mu}^{b} \zeta^{c}=D_{\mathrm{A}_{\mu}} \zeta^{a}
$$

for which the connection 1-form is $\kappa_{\mathrm{YM}} a^{a}{ }_{b c} A_{\mu}^{b}$. The associated curvature $\left[D_{\mathrm{A}}, D_{\mathrm{A}}\right]$ of this connection yields the Yang-Mills field strength 2-form

$$
F_{\mathrm{A}}^{a}{ }_{\mu \nu}=\partial_{[\mu} A_{\nu]}^{a}+\frac{1}{2} \kappa_{\mathrm{YM}} a_{b c}^{a} A_{\mu}^{b}
$$

which satisfies the Bianchi divergence identity

$$
D_{\mathrm{A}}^{\mu} * F_{\mathrm{A}_{\mu}}^{a}=0
$$

due to $\left[D_{\mathrm{A}},\left[D_{\mathrm{A}}, D_{\mathrm{A}}\right]\right]=0$. The field equation in three dimensions is given by the covariant curl of the dual field strength

$$
E_{\mu}^{\mathrm{YM} a}=\epsilon_{\mu}^{\nu \alpha} D_{\mathrm{A}_{\nu}} * F_{\mathrm{A}_{\alpha}}^{a}=0
$$

arising from the Lagrangian

$$
L_{\mathrm{YM}}=\frac{1}{2} g^{\mu \nu} k_{a b} * F_{\mathrm{A}_{\mu}}^{a} * F_{\mathrm{A}_{\nu}}^{b}
$$

The gauge group generated by $\delta_{\zeta}^{\mathrm{YM}}$ is an infinite-dimensional representation of $\mathfrak{g}$,

$$
\left[\delta_{\zeta_{1}}^{\mathrm{YM}}, \delta_{\zeta_{2}}^{\mathrm{YM}}\right]=\delta_{\zeta_{3}}^{\mathrm{YM}}, \quad \zeta_{3}=\left[\zeta_{1}, \zeta_{2}\right]
$$

where $\left[\zeta_{1}, \zeta_{2}\right]$ denotes the Lie algebra commutator, i.e. $\zeta_{3}{ }^{a}=a^{a}{ }_{b c} \zeta_{1}{ }^{b} \zeta_{2}{ }^{c}$.

By comparison, in Freedman-Townsend theory the main geometrical role is played by the dual field strength

$$
K_{\mu}^{a}=Y_{\mathrm{A} \mu b}^{-1 a \nu} * F_{\nu}^{b}
$$

as defined using the inverse of the symmetric linear map $Y_{\mathrm{A}}$ on Lie-algebra valued 1-forms

$$
Y_{\mathrm{A} \mu b}^{a \nu}=\delta_{a}^{b} \delta_{\nu}^{\mu}-\kappa_{\mathrm{FT}} b_{b c}^{a}{ }_{\mu}{ }^{\nu \sigma} A_{\alpha}^{c}
$$


(note the inverse exists as a well-defined power series whenever $A$ is restricted such that $\left.\operatorname{det} Y_{\mathrm{A}} \neq 0\right)$. This field strength appears as a connection 1 -form $b^{a}{ }_{b c} K_{\mu}^{b}$ introduced through the gauge symmetry

$$
\delta_{\zeta}^{\mathrm{FT}} A_{\mu}^{a}=\partial_{\mu} \zeta^{a}+\kappa_{\mathrm{FT}} b_{b c}^{a} K_{\mu}^{b} \zeta^{c}=D_{\mathrm{K}_{\mu}} \zeta^{a}
$$

which defines a covariant derivative $D_{\mathrm{K}}$. Geometrically, the potential $A_{\mu}^{a}$ is related to the connection by a covariant curl

$$
\epsilon_{\mu}^{\nu \alpha} D_{\mathrm{K}_{\nu}} A_{\alpha}^{a}=K_{\mu}^{a}
$$

The field equation is given by the dual of the associated curvature 2-form

$$
E_{\mu}^{\mathrm{FT} a}=\epsilon_{\mu}^{\nu \alpha}\left(\partial_{\nu} K_{\alpha}^{a}+\frac{1}{2} \kappa_{\mathrm{FT}} b_{b c}^{a} K_{\nu}^{b} K_{\alpha}^{c}\right)=* R_{\mathrm{K}_{\mu}}^{a}=0
$$

and arises from the Lagrangian

$$
L_{\mathrm{FT}}=\frac{1}{2} g^{\mu \nu} K_{\mu}^{a} * F_{\nu}^{b}=\frac{1}{2} Y_{\mathrm{A}}^{\mu \nu} K_{\mu}^{a} K_{\nu}^{b}
$$

with $Y_{\mathrm{A}_{a b}^{\mu \nu}}^{\mu \nu}=k_{a c} g^{\mu \alpha} Y_{\mathrm{A}_{\alpha b}}^{c \nu}$ denoting the quadratic form corresponding to the linear map (3.28). This Lagrangian is gauge invariant to within a divergence. The gauge symmetry generates a gauge group that is abelian on the solution space

$$
\left.\left[\delta_{\zeta_{1}}^{\mathrm{FT}}, \delta_{\zeta_{2}}^{\mathrm{FT}}\right]\right|_{E^{\mathrm{FT}}=0}=0
$$

Due to the abelian Bianchi identity $\partial^{\mu} * F_{\mu}^{a}=0$, the field strength satisfies a divergence identity

$$
\partial^{\mu} K_{\mu}^{a}=0
$$

for solutions. Moreover, the curvature $R_{\mathrm{K}}$ vanishes on solutions, so $K$ is a flat connection. Hence (if $M$ is assumed to be a simply connected manifold, on which $\left(\mathbb{V}, b_{b c}{ }^{a}\right)$ is viewed as a trivial bundle $\mathbb{V} \times M)$, the connection 1 -form is given by a chiral field strength

$$
K_{\mu}^{a}=\left(U^{-1} \partial_{\mu} U\right)^{a}
$$

where $U$ is a map from spacetime $M$ into the simply-connected semisimple Lie group $\mathcal{G}$ whose Lie algebra is $\mathfrak{g}$, with the tangent space of the group manifold at the identity element identified with the Lie algebra. As is customary, this map will be taken to be a principal 
matrix representation of the Lie group, so thus $U$ is a matrix-valued function. The divergence identity on $K$ now implies $U$ satisfies the massless chiral field equation

$$
\partial^{\mu}\left(U^{-1} \partial_{\mu} U\right)=0 \text { or equivalently } \square U=\partial^{\mu} U U^{-1} \partial_{\mu} U
$$

In physical terms, solutions $U$ describe a nonlinearly coupled set of massless spin-zero (matrix) fields.

One interesting feature common to both theories is a geometrical relation linking the gauge symmetry, field strength, and action of the gauge group through the covariant derivative operator, given by

$$
\begin{aligned}
& D_{\mathrm{A}_{[\mu}} \delta_{\zeta}^{\mathrm{YM}} A_{\nu]}^{a}=a^{a}{ }_{b c} F_{\mathrm{A}_{\mu \nu}}^{b} \zeta^{c}=\delta_{\zeta}^{\mathrm{YM}} F_{\mathrm{A}_{\mu \nu}}^{a} \text {, } \\
& D_{\mathrm{K}_{[\mu}} \delta_{\zeta}^{\mathrm{FT}} A_{\nu]}^{a}=b^{a}{ }_{b c} R_{\mathrm{K}_{\mu \nu}}^{b} \zeta^{c}=\epsilon_{\mu \nu}{ }^{\alpha} Y_{\mathrm{A}_{\alpha b}}{ }^{a}{ }_{\zeta}{ }_{\zeta}^{\mathrm{FT}} K_{\beta}^{b},
\end{aligned}
$$

in accordance with the deformation theorem 2.8 in Sec. II.

The combined deformation with nonabelian Chern-Simons term mixes separate nonlinear aspects of Yang-Mills and Freedman-Townsend theories in an interesting way. To proceed with the construction of the deformation to all orders, first put $\kappa_{\mathrm{FT}}=m^{-1} \kappa_{\mathrm{YM}}$ and replace $* F$ in the Freedman-Townsend field strength (3.27) by the Yang-Mills field strength $* F_{\mathrm{A}}$,

$$
K_{\mu}^{a}=Y_{\mathrm{A} \mu b}^{-1 a \nu} * F_{\mathrm{A}_{\nu}}^{b}
$$

where $Y_{\mathrm{A}}$ is unchanged. In terms of this nonpolynomial field strength, the combined YangMills and Freedman-Townsend gauge symmetry in the deformation is given by

$$
\delta_{\zeta} A_{\mu}^{a}=\partial_{\mu} \zeta^{a}+\kappa_{\mathrm{YM}} a_{b c}^{a}\left(A_{\mu}^{b}+m^{-1} K_{\mu}^{b}\right) \zeta^{c}
$$

The deformed Lagrangian is obtained by directly combining the Chern-Simons Lagrangian and the Freedman-Townsend Lagrangian for the deformed field strength

$$
L=\frac{1}{2} K_{\mu}^{a} K_{\nu}^{b} Y_{\mathrm{A}_{a b}}^{\mu \nu}+\frac{1}{2} m \kappa_{\mathrm{YM}} a_{a b c} \epsilon^{\alpha \mu \nu} A_{\alpha}^{a}\left(\partial_{\mu} A_{\nu}^{b}+\frac{1}{3} a_{c d}^{b} A_{\mu}^{c} A_{\nu}^{d}\right) .
$$

Substitution of the field strength $F_{\mathrm{A}}=Y_{\mathrm{A}} K$ into the Chern-Simons term yields the equivalent expression

$$
L=\frac{1}{2} g^{\mu \nu} k_{a b} K_{\nu}^{b}\left(m A_{\mu}^{a}+K_{\mu}^{a}\right)-\frac{1}{2} \kappa_{\mathrm{YM}} m^{-1} a_{a b c} \epsilon^{\mu \nu \alpha}\left(\left(m A_{\mu}^{a}+K_{\mu}^{a}\right) K_{\nu}^{b}+m^{2} \frac{1}{6} A_{\mu}^{a} A_{\nu}^{b}\right) A_{\alpha}^{c} .
$$


This Lagrangian is invariant to within a divergence under the gauge symmetry (3.40),

$$
\delta_{\zeta} L=\partial_{\mu}\left(\frac{1}{2} \epsilon^{\mu \alpha \nu} a_{a b c}\left(A_{\nu}^{a}\left(m A_{\alpha}^{a}+K_{\alpha}^{a}\right)+m^{-1} K_{\nu}^{a} K_{\alpha}^{b}\right) \zeta^{c}+m g^{\mu \nu} k_{a b} * F_{\mathrm{A}_{\nu}}^{a} \zeta^{b}\right) .
$$

The field equation obtained from the Euler-Lagrange operator applied to $L$ is given by a Chern-Simons term plus a Freedman-Townsend curvature deformed with an extra Yang-Mills coupling

$$
E_{a}^{\mu}=g^{\mu \nu} k_{a b}\left(m * F_{\nu}^{b}+\epsilon_{\nu}^{\alpha \beta}\left(\partial_{\alpha} K_{\beta}^{b}+\kappa_{\mathrm{YM}} \frac{1}{2} a_{c d}^{b} K_{\alpha}^{c}\left(m^{-1} K_{\beta}^{d}+2 A_{\beta}^{d}\right)\right)\right)=0 .
$$

Through the relation between the Yang-Mills and Freedman-Townsend field strengths, the field equation simplifies elegantly to

$$
\epsilon_{\nu}^{\alpha \beta}\left(\partial_{\alpha} K_{\beta}^{b}+\kappa_{\mathrm{YM}} m^{-1} \frac{1}{2} a^{a}{ }_{c d} K_{\alpha}^{c} K_{\beta}^{d}\right)+m K_{\mu}^{a}=0
$$

which involves only the Freedman-Townsend field strength itself. The Bianchi identity on $* F_{\mathrm{A}}$ leads to a covariant divergence identity on this field strength

$$
g^{\mu \nu}\left(D_{\mathrm{K}_{\mu}} K_{\nu}^{a}-b^{a}{ }_{b c} E_{\mu}^{b} A_{\nu}^{c}\right)=0 .
$$

Since the Lie algebra is semisimple, this identity reduces to an ordinary divergence

$$
\partial^{\mu} K_{\mu}^{a}=0
$$

holding on solutions of the field equation.

\section{B. Geometrical structure}

There is a highly interesting geometrical structure behind this nonlinear generalization of Yang-Mills Chern-Simons gauge theory, blending features of pure Yang-Mills theory and pure Freedman-Townsend theory involving their connections and associated covariant derivatives

and curvatures. It will be useful to employ an index-free notation: $\mathbf{A}=A_{\mu}^{a} d x^{\mu} \mathbf{e}_{a}$ and $\mathbf{K}=K_{\mu}^{a} d x^{\mu} \mathbf{e}_{a}$ are $\mathfrak{g}$-valued 1-forms, where $\mathbf{e}_{a}$ denotes a fixed basis for $\mathfrak{g}$; likewise $\boldsymbol{\zeta}=\zeta^{a} \mathbf{e}_{a}$ is $\mathfrak{g}$-valued function. Hereafter, $\kappa_{\mathrm{YM}}=m \kappa_{\mathrm{FT}}=1$ for simplicity.

First, the potential $\mathbf{A}$ is related to the field strength $\mathbf{K}$ by a deformed Yang-Mills curvature of $\mathbf{D}_{A}$

$$
-* \mathbf{K}=\mathbf{D}_{\frac{1}{m} K} \mathbf{A}+\frac{1}{2}[\mathbf{A}, \mathbf{A}]
$$


in which the Freedman-Townsend covariant derivative $\mathbf{D}_{\frac{1}{m} K}$ replaces an ordinary exterior derivative. The field equation relates the curvature of $\mathbf{D}_{\frac{1}{m} K}$ to $\mathbf{K}$ itself,

$$
* \mathbf{R}_{\frac{1}{m} K}=-\mathbf{K}
$$

The Bianchi identity associated with this curvature $0=\mathbf{D}_{\frac{1}{m} K} \mathbf{R}_{\frac{1}{m} K}=\mathbf{D}_{\frac{1}{m} K} * \mathbf{K}$ reduces to an ordinary (abelian) zero curl equation

$$
d * \mathbf{K}=0
$$

due to the algebraic identity $[\mathbf{K}, * \mathbf{K}]=0$. Surprisingly, the curvature and curl equations (3.49) and (3.50) together constitute a massive nonabelian Chern-Simons Proca theory for the potential $\mathbf{A}_{\text {mass. }}=m^{-1} \mathbf{K}$ in Lorentz gauge,

$$
\operatorname{div} \mathbf{A}_{\text {mass. }}=0, \quad * \mathbf{F}_{\text {Amass }}=-m \mathbf{A}_{\text {mass. }},
$$

whose Lagrangian is

$$
\boldsymbol{L}_{\mathrm{CS}}+\boldsymbol{L}_{\mathrm{m}}=\frac{1}{2} \operatorname{tr}\left(\mathbf{A}_{\text {mass. }} \wedge d \mathbf{A}_{\text {mass. }}+\frac{1}{3} \mathbf{A}_{\text {mass. }} \wedge\left[\mathbf{A}_{\text {mass. }}, \mathbf{A}_{\text {mass. }}\right]+m^{2} \mathbf{A}_{\text {mass. }} \wedge * \mathbf{A}_{\text {mass. }}\right)
$$

where tr denotes the inner product with respect to the Cartan-Killing metric on $\mathfrak{g}$. The physical content of this theory thus describes a massive spin-one nonabelian vector field A mass.

Second, the gauge symmetry defines a covariant derivative operator

$$
\delta_{\zeta} \mathbf{A}=\mathbf{D}_{A} \boldsymbol{\zeta}+m^{-1}[\mathbf{K}, \boldsymbol{\zeta}]=\mathbf{D}_{K} \boldsymbol{\zeta}+[\mathbf{A}, \boldsymbol{\zeta}]=\mathbf{D}_{A+\frac{1}{m} K} \boldsymbol{\zeta}
$$

based on a combined connection 1-form $\operatorname{ad}_{A+\frac{1}{m} K}=\left[\mathbf{A}+m^{-1} \mathbf{K}, \cdot\right]$. Through the field equation, the curvature of this connection is zero

$$
\mathbf{R}_{A+\frac{1}{m} K}=0
$$

on solutions A. Since in addition this connection transforms in the expected way under the gauge group on solutions,

$$
\delta_{\zeta}\left(\mathbf{A}+m^{-1} \mathbf{K}\right)=\mathbf{D}_{A+\frac{1}{m} K} \boldsymbol{\zeta}
$$

Lorentz gauge can be imposed

$$
\operatorname{div}\left(\mathbf{A}+m^{-1} \mathbf{K}\right)=0
$$


(which is achieved by a suitable finite gauge transformation, analogously to Yang-Mills theory). Then these equations (3.54) and (3.56) are identical with the field strength equations in pure Freedman-Townsend theory for $\mathbf{K}_{\text {flat }}=\mathbf{A}+m^{-1} \mathbf{K}$,

$$
\mathbf{R}_{K_{\text {flat }}}=0, \quad \operatorname{div} \mathbf{K}_{\text {flat }}=0 .
$$

As a result, $\mathbf{K}_{\text {flat }}$ is a flat connection and hence is given by

$$
\mathbf{K}_{\text {flat }}=U^{-1} d U
$$

in terms of a chiral field $U$ introduced as in pure Freedman-Townsend theory, namely $U$ is a function from spacetime into the simply-connected Lie group of matrices whose Lie algebra is $\mathfrak{g}$. Since $U$ satisfies the massless spin-zero chiral equation, the potential A has the decomposition

$$
\mathbf{A}=U^{-1} d U-\mathbf{A}_{\text {mass }}
$$

given by a massive spin-one part $\mathbf{A}_{\text {mass. }}$ satisfying Lorentz gauge and a massless spin-zero part $U^{-1} d U$ representing the residual gauge freedom left in Lorentz gauge. Interestingly, if Lorentz gauge is relaxed on $\mathbf{A}$, then $U$ becomes coupled as a pure gauge field to $\mathbf{A}_{\text {mass. }}$, as will be seen in Sec. IV when the torsion generalization of this theory is discussed.

Thus, the nonlinear generalization of Yang-Mills Chern-Simons gauge theory possesses a striking geometrical duality with pure massless chiral field theory combined with massive Chern-Simons gauge theory.

Lastly, in conformance with the deformation theorem 2.8, the gauge symmetry and field strength are linked by the geometrical relation

$$
\mathbf{D}_{A+\frac{1}{m} K} \delta_{\zeta} \mathbf{A}=\left[\mathbf{R}_{A+\frac{1}{m} K}, \boldsymbol{\zeta}\right]=\mathbf{Y}_{A}\left(\delta_{\zeta} \mathbf{K}\right)
$$

where $\mathbf{Y}_{A}=\mathbf{1}-\frac{1}{m} *[\mathbf{A}, \cdot]$ is the linear map on $\mathfrak{g}$-valued 1-forms.

\section{TORSION GENERALIZATION OF YANG-MILLS CHERN-SIMONS GAUGE THEORY}

A natural setting for torsion is provided through the duality of Freedman-Townsend gauge theory and chiral field theory for a general semisimple Lie group $\mathcal{G}$ with Lie algebra 
$\mathfrak{g}=\left(\mathbb{V}, c_{b c}^{a}\right)$. To express this duality at the Lagrangian level, it is convenient to pass to a 1st order formalism for the Freedman-Townsend Lagrangian

$$
L_{\mathrm{FT}}^{1 s t}=g^{\mu \nu} k_{a b}\left(A_{\mu}^{a} * R_{\mathrm{K}_{\nu}}^{b}-\frac{1}{2} K_{\mu}^{a} K_{\nu}^{b}\right)
$$

where the Lie-algebra valued potential $A_{\mu}^{a}$ and field strength $K_{\mu}^{a}$ are treated as independent field variables. The respective field equations yield the curl equation (3.30) relating $A_{\mu}^{a}$ to $K_{\mu}^{a}$, and the zero curvature equation (3.31) on $K_{\mu}^{a}$. Note that a substitution of $K_{\mu}^{a}$ into $L_{\mathrm{FT}}^{1 s t}$ through the nonpolynomial relation (3.27) in terms of $A_{\mu}^{a}$ recovers the Freedman-Townsend Lagrangian (3.32). A dual formulation is obtained if instead $A_{\mu}^{a}$ is eliminated by substituting a zero-curvature expression (3.35) for $K_{\mu}^{a}$ into $L_{\mathrm{FT}}^{1 s t}$ in terms of a chiral field. To do this, it is useful geometrically to view the Lie group $\mathcal{G}$ in the well-known way [42] as a Riemannian symmetric space whose $\mathcal{G}$-invariant metric is the Cartan-Killing tensor $k_{A B}$ on the Lie group and to write the chiral field as a map $\varphi^{A}$ from spacetime into $\mathcal{G}$, using local coordinates (denoted by upper case latin indices) for the group manifold. Consider a left-invariant orthonormal frame $e_{a}^{A}$ on this space, satisfying the commutator relation

$$
\left[e_{a}, e_{b}\right]^{B}=2 e_{[a}^{A} \partial_{A} e_{b]}^{B}=\kappa_{\mathrm{FT}} e_{c}^{B} c_{a b}^{c}
$$

and the orthonormality relation

$$
e_{a}^{A} e_{b}^{B} k_{A B}=k_{a b}
$$

where $c^{c}{ }_{a b}$ is the structure tensor of the Lie algebra and $k_{a b}=-c^{c}{ }_{a d} c^{d}{ }_{b c}$ is the Cartan-Killing metric. By means of a corresponding coframe $e_{A}^{a}$, which is a left-invariant Lie-algebra valued 1-form on $\mathcal{G}$, the zero-curvature Freedman-Townsend field strength is given by

$$
K_{\mu}^{a}=e_{A}^{a}(\varphi) \partial_{\mu} \varphi^{A}
$$

Here $e_{A}^{a}(\varphi)$ denotes the coframe evaluated at the point in the group manifold given by the chiral field $\varphi^{A}$. Substitution of $K_{\mu}^{a}$ into $L_{\mathrm{FT}}^{1 s t}$ then gives the chiral theory Lagrangian

$$
L_{\text {chiral }}=-\frac{1}{2} g^{\mu \nu} k_{A B}(\varphi) \partial_{\mu} \varphi^{A} \partial_{\nu} \varphi^{B}=-\frac{1}{2} g^{\mu \nu} k_{a b} e_{A}^{a}(\varphi) \partial_{\mu} \varphi^{A} e_{B}^{b}(\varphi) \partial_{\nu} \varphi^{B}
$$

with the standard field equation

$$
E_{\text {chiral }}^{A}=g^{\mu \nu}\left(\partial_{\mu} \partial_{\nu} \varphi^{A}+\Gamma_{B C}^{A}(\varphi) \partial_{\mu} \varphi^{B} \partial_{\nu} \varphi^{C}\right)=0
$$


where

$$
\Gamma_{B C}^{A}=g^{A D}\left(\partial_{(B} k_{C) D}-\frac{1}{2} \partial_{D} k_{B C}\right)
$$

is the Christoffel symbol of the Lie group Cartan-Killing metric $k_{A B}$. Geometrically, $\Gamma_{B C}^{A}$ describes a symmetric Riemannian connection determined by this metric. Following the approach in Ref. 33], torsion will now be added to the theory by a geometrical term in the Lagrangian of the form

$$
L_{\text {tors. }}=-\frac{1}{2} v^{\mu \nu} p_{A B}(\varphi) \partial_{\mu} \varphi^{A} \partial_{\nu} \varphi^{B}
$$

which involves introducing a constant skew tensor $v^{\mu \nu}$ on spacetime and a left-invariant 2 -form $p_{A B}$ on the Lie group. This contributes a torsion term to the chiral field equation

$$
E_{\text {tors. }}^{A}=v^{\mu \nu} Q_{B C}^{A}(\varphi) \partial_{\mu} \varphi^{B} \partial_{\nu} \varphi^{C}
$$

where

$$
Q_{B C}^{A}=-\frac{3}{2} k^{A D} \partial_{[D} p_{B C]}
$$

which serves geometrically as torsion added into the connection on the Lie group. The field equation with torsion is thus given by

$$
E_{\text {chiral }}^{A}+E_{\text {tors. }}^{A}=\left(g^{\mu \nu}+v^{\mu \nu}\right)\left(\partial_{\mu} \partial_{\nu} \varphi^{A}+\left(\Gamma_{B C}^{A}(\varphi)+Q_{B C}^{A}(\varphi)\right) \partial_{\mu} \varphi^{B} \partial_{\nu} \varphi^{C}\right)=0 .
$$

Because the torsion potential $p_{B C}$ is $\mathcal{G}$-invariant, it has a well defined pull back to the Lie algebra, so

$$
p_{a b}=e_{a}^{A} e_{b}^{B} p_{A B}
$$

is a constant tensor as will be necessary for torsion to be compatible with duality. Then, through the relation

$$
c^{c}{ }_{a b}=-2 e_{a}^{A} e_{b}^{B} \partial_{[A} e_{B]}^{c}
$$

derived from the commutator (4.2), the torsion in the connection leads to a corresponding constant tensor

$$
Q^{a}{ }_{b c}=\frac{3}{2} k^{a e} p_{d\left[e^{c}{ }^{c}{ }_{b c]}\right.}=e_{A}^{a} e_{b}^{B} e_{c}^{C} Q_{B C}^{A}
$$

in terms of the metric $k_{a b}$ and torsion potential $p_{a b}$ on $\mathbb{V}$. This allows the torsion Lagrangian (4.8) to be expressed entirely in terms of the field strength (4.4) by

$$
L_{\text {tors. }}^{1 s t}=-\frac{1}{2} v^{\mu \nu} p_{a b} K_{\mu}^{a} K_{\nu}^{b}
$$


A dual formulation of the torsion chiral theory Lagrangian $L_{\text {chiral }}+L_{\text {tors. }}$ is now obtained simply by adding $L_{\text {tors. }}^{1 s t}$ to the 1 st order form of the Freedman-Townsend Lagrangian $L_{\text {tors. }}^{1 s t}$. This yields

$$
L_{\mathrm{FT}}^{1 s t}+L_{\text {tors. }}^{1 s t}=g^{\mu \nu} k_{a b} A_{\mu}^{a} * R_{\mathrm{K}_{\nu}}^{b}-\frac{1}{2} \tilde{g}_{a b}^{\mu \nu} K_{\mu}^{a} K_{\nu}^{b}
$$

where it is convenient to introduce

$$
\tilde{g}_{a b}^{\mu \nu}=g^{\mu \nu} k_{a b}+v^{\mu \nu} p_{a b}
$$

which defines a symmetric inner product on Lie-algebra valued 1-forms. The effect of torsion in this Lagrangian is to generalize the field equation of $K_{\mu}^{a}$ so it becomes

$$
K_{\mu}^{a}=\tilde{Y}_{\mathrm{A} \mu b}^{-1 a \nu} * F_{\nu}^{b}
$$

using the inverse of the linear map $\tilde{Y}_{\mathrm{A}}$ covariantly constructed in terms of the potential $A_{\mu}^{a}$, where

$$
\tilde{Y}_{\mathrm{A}_{a b}^{\mu \nu}}^{\mu \nu} \tilde{g}_{a b}^{\mu \nu}-\epsilon^{\mu \nu \alpha} \kappa_{\mathrm{FT}} c_{a b c} A_{\alpha}^{c}=\tilde{Y}_{\mathrm{A}_{\alpha b}^{c \mu} g^{\mu \alpha} k_{a c}}
$$

is the corresponding quadratic form on Lie-algebra valued 1-forms. A simple algebraic analysis shows that the inner product (4.17) is nondegenerate, so the linear map

$$
\tilde{Y}_{\mathrm{A}}^{a \nu b}=\delta_{b}^{a} \delta_{\mu}^{\nu}+p^{a}{ }_{b} v_{\mu}^{\nu}-\epsilon_{\mu}^{\nu \alpha} \kappa_{\mathrm{FT}} c^{a}{ }_{b c} A_{\alpha}^{c}
$$

is invertible by a power series in $A$ (under the restriction $\operatorname{det} \tilde{Y}_{\mathrm{A}} \neq 0$ ), with $p^{a}{ }_{b}=k^{a c} p_{c b}$ and $v_{\mu}{ }^{\nu}=g_{\mu \alpha} v^{\alpha \nu}$. Substitution of the field strength (4.18) back into $L_{\mathrm{FT}}^{1 s t}+L_{\text {tors. }}^{1 s t}$ then yields a torsion generalization of Freedman-Townsend gauge theory

$$
L_{\mathrm{FT}}^{\mathrm{tors}}=\tilde{g}_{a b}^{\mu \nu} K_{\mu}^{a} K_{\nu}^{b}=\tilde{g}_{a b}^{-1 \mu \nu} * F_{\mu}^{a} * F_{\nu}^{b}
$$

In this generalization, the Lagrangian remains gauge-invariant to within a divergence, such that the gauge symmetry has the same form given by the covariant derivative of the gauge parameter $\zeta^{a}$ using $K_{\mu}^{a}$ as a Lie-algebra valued connection 1-form. This is an immediate consequence of the gauge invariance of $L_{\mathrm{FT}}^{1 s t}$ and $L_{\text {tors. }}^{1 s t}$ under $\delta_{\zeta}^{1 s t} K_{\mu}^{a}=0, \delta_{\zeta}^{1 s t} A_{\mu}^{a}=D_{\mathrm{K}_{\mu}} \zeta^{a}$. Moreover, from the form of $L_{\mathrm{FT}}^{1 s t}$, the field equation coming from $L_{\mathrm{FT}}^{\text {tors. }}$ retains the form of a zero-curvature equation on $K_{\mu}^{a}$. The only place where torsion enters explicitly is in the divergence equation on $K_{\mu}^{a}$

$$
\partial^{\mu} K_{\mu}^{a}+Q_{b c}^{a} v^{\mu \nu} K_{\mu}^{b} K_{\nu}^{c}=R_{\mathrm{K}_{\mu \nu}}^{b}\left(\epsilon^{\mu \nu \alpha} \kappa_{\mathrm{FT}} c^{a}{ }_{b c} A_{\alpha}^{c}-v^{\mu \nu} p^{a}{ }_{b}\right)
$$


derived through the identity $0=\partial^{\mu} * F_{\mu}^{a}=\tilde{g}_{\mu b}^{a \nu} \partial^{\mu} K_{\nu}^{b}$. On solutions of the field equation, this divergence equation reduces precisely to the dual form of the torsion chiral field equation (3.36).

Finally, it is worth noting that the algebraic formulation (4.14) of torsion in the Lie algebra gives rise to a slight restriction on the dimension of the Lie group [33].

Proposition 4.1: For semisimple Lie groups $\mathcal{G}$, if the dimension of $\mathcal{G}$ is three, then every torsion potential has the form $p_{b c}=p_{d} c^{d}{ }_{b c}$ for some vector $p_{d}$ in the Lie algebra and hence the torsion vanishes by the Jacobi identity. This is not the case if $\mathcal{G}$ has dimension larger than three. In particular, every such Lie group possesses an abelian subalgebra of dimension at least two, allowing a non-zero torsion tensor $Q^{a}{ }_{b c}$ to exist with a potential given by the bi-vector form $p_{a b}=2 p_{[a} q_{b]}$, where $p_{a}, q_{a}$ are any two (linearly independent) commuting vectors in the Lie algebra.

\section{A. Linear abelian theory with torsion}

The main goal now will be to derive a Yang-Mills generalization of the FreedmanTownsend gauge theory with torsion, in the setting of a deformation analysis as follows. To begin, consider a linearization of the torsion Freedman-Townsend theory around the zero potential $A_{\mu}^{a}=0$ by turning off the Freedman-Townsend coupling $\kappa_{\mathrm{FT}}=0$. This gives a novel abelian gauge theory with torsion for the potential $A_{\mu}^{a}$. The gauge-invariant quadratic Lagrangian takes the form

$$
\stackrel{(2)}{L}=\tilde{g}_{a b}^{-1 \mu \nu} * F_{\mu}^{a} * F_{\nu}^{b}
$$

where $\tilde{g}_{a b}^{-1 \mu \nu}$ denotes the inverse of the nondegenerate symmetric inner product (4.17) with respect to the spacetime metric $g_{\mu \nu}$ and the internal vector space metric $k_{a b}$, such that $\tilde{g}_{a c}^{-1 \mu \alpha} g_{\alpha \beta} k^{c d} \tilde{g}_{d b}^{-1 \beta \nu}=g^{\mu \nu} k_{a b}$. There is no change to the form of the abelian gauge symmetry, $\stackrel{(0)}{\delta}_{\zeta} A_{\mu}^{a}=\partial_{\mu} \zeta^{a}$. The linear field equation of this theory

$$
\stackrel{(1)}{E}_{a}^{\mu}=-\epsilon_{\alpha}{ }^{\nu \mu} \partial_{\nu} * \tilde{F}_{a}^{\alpha}
$$

is given by the curl of the gauge-invariant torsion field strength

$$
* \tilde{F}_{a}^{\mu}=\tilde{g}_{a b}^{-1 \mu \nu} * F_{\nu}^{b}=\tilde{g}_{a b}^{-1 \mu \nu} \epsilon_{\nu}{ }^{\alpha \beta} \partial_{\alpha} A_{\beta}^{b} .
$$


It is interesting to include an abelian Chern-Simons term in the theory by adding the Lagrangian $\stackrel{(2)}{L}_{\mathrm{CS}}$. This contributes the same linear Chern-Simons term to the field equation as in the case without torsion, which now gives

$$
\stackrel{(1)}{E} \underset{a}{\mathrm{CS} \mu}=m g^{\mu \nu} k_{a b} * F_{\nu}^{b}=m \tilde{g}_{a \nu}^{\mu b} * \tilde{F}_{b}^{\nu}=m * \tilde{F}_{a}^{\mu}+m v_{\nu}{ }^{\mu} p^{b}{ }_{a} * \tilde{F}_{b}^{\nu}
$$

after inverting the field strength relation (4.25). Thus, $A_{\mu}^{a}$ satisfies the gauge-invariant field equation $\stackrel{(1)}{E_{a}^{\mu}}+\stackrel{(1)}{E} \underset{a}{\mathrm{CS} \mu}=0$.

To understand the physical meaning of torsion in this theory, it is useful to look at the equations satisfied by the gauge-invariant field strength $* \tilde{F}_{a}^{\mu}$. First write the skew tensor

$$
v^{\mu \nu}=\epsilon^{\mu \nu \alpha} v_{\alpha}
$$

by introducing the dual vector $v_{\alpha}$ on spacetime. Note this vector represents an extra background structure introduced on spacetime, which breaks Lorentz covariance. Then the field equation $\stackrel{(1)}{E}_{a}^{\mu}+\stackrel{(1)}{E} \underset{a}{\mathrm{CS} \mu}=0$ has the explicit non-covariant form

$$
\epsilon_{\alpha}{ }^{\nu \mu} \partial_{\nu} * \tilde{F}_{a}^{\alpha}=m * \tilde{F}_{a}^{\mu}-m p_{a}^{b} \epsilon_{\alpha}{ }^{\nu \mu} v_{\nu} * \tilde{F}_{b}^{\alpha}
$$

Next the abelian Bianchi identity $0=\partial^{\mu} * F_{\mu}^{a}=\tilde{g}_{\nu a}^{b \mu} \partial_{\mu} * \tilde{F}_{b}^{\nu}$ gives the divergence equation

$$
\partial_{\mu} * \tilde{F}_{a}^{\mu}=-m p_{a}^{b} v_{\mu} * \tilde{F}_{b}^{\mu}
$$

after simplifications of the curl term through the field equation. These curl and divergence equations on $* \tilde{F}_{a}^{\mu}$ have a more elegant form if expressed via the derivative operator

$$
\tilde{\partial}_{\mu}=\partial_{\mu}+m p^{b}{ }_{a} v_{\mu}
$$

based on a connection $m p^{b}{ }_{a} v_{\mu}$ for Lie-algebra valued differential forms on $M$. Because $v_{\mu}$ and $p_{a}^{b}$ are constant on $M$ and have vanishing exterior product, this connection is flat. Thus, the field strength $* \tilde{F}_{a}^{\mu}$ satisfies

$$
\tilde{\partial}_{\mu} * \tilde{F}_{a}^{\mu}=0, \quad \epsilon_{\alpha}^{\beta \mu} \tilde{\partial}_{\beta} * \tilde{F}_{a}^{\alpha}=-m * \tilde{F}_{a}^{\mu},
$$

which differ from the standard field strength equations only by the torsion parts in the definition of $* \tilde{F}_{a}^{\mu}$ and $\tilde{\partial}_{\mu}$.

Consequently, as in the case without torsion, a further curl applied to the curl equation on the field strength yields a vector wave equation

$$
\widetilde{\square} * \tilde{F}_{a}^{\mu}=m^{2} * \tilde{F}_{a}^{\mu}
$$


and hence the torsion field strength physically describes a set of free spin-one fields of mass $m$, each with a single dynamical degree of freedom. In the massless case $m=0$, the torsion does not explicitly appear in this wave equation, so the spin-one vector fields $* \tilde{F}_{a}^{\mu}$ have the same propagation as in the torsionless covariant theory, $\square * \tilde{F}_{a}^{\mu}=0$. But in the massive case $m \neq 0$, the corresponding propagation is altered by a novel non-covariant "twist" effect due to the connection term $m p_{a}^{b} v_{\mu}$ in the derivative operator $\tilde{\partial}_{\mu}$ as result of the torsion. More precisely, for plane wave solutions $* \tilde{F}_{a}^{\mu}=\varepsilon_{a}^{\mu} \exp \left(\mathrm{i} k_{\alpha} x^{\alpha}\right)$, the polarization $\varepsilon_{a}^{\mu}=$ const. is found to be internally aligned relative to the eigenvectors of $p^{b}{ }_{a}$, while the mass shell condition on the propagation vector $k_{\alpha}=$ const. is found to undergo a shift proportional to $v_{\mu}$ determined by the eigenvalues of $p_{a}^{b}$.

The field-theoretic content of torsion is best seen by passing to the dual form of the free Lagrangian $\stackrel{(2)}{L}+\stackrel{(2)}{L}_{\mathrm{CS}}$. Observe the terms in the linear field equation (4.28) have the form of a curl, $\stackrel{(1)}{E}_{a}^{\mu}+\stackrel{(1)}{E}_{a}^{\mathrm{CS} \mu}=\epsilon^{\mu \nu \alpha} k_{a b} \partial_{\nu}\left(* \tilde{F}_{\alpha}^{b}+m A_{\alpha}^{b}\right)$. So, for solutions $A_{\mu}^{a}$,

$$
* \tilde{F}_{\mu}^{a}+m A_{\mu}^{a}=\partial_{\mu} \varphi^{a}
$$

is a gradient. Here, the $\mathbb{V}$-valued scalar field $\varphi^{a}=e_{A}^{a} \varphi^{A}$ geometrically represents an abelian chiral field in a left-invariant frame $e_{A}^{a}$ on the abelian Lie group whose Lie algebra is identified with the vector space $\mathbb{V}$. Elimination of the torsion field strength $* \tilde{F}_{\mu}^{a}$ from $\stackrel{(2)}{L}+\stackrel{(2)}{L}_{\mathrm{CS}}$ leads to the equivalent dual Lagrangian

$$
\stackrel{(2)}{L}_{\text {dual }}=-\frac{1}{2} \tilde{g}_{a b}^{-1 \mu \nu}\left(\partial_{\mu} \varphi^{a}-m A_{\mu}^{a}\right)\left(\partial_{\nu} \varphi^{a}-m A_{\nu}^{a}\right)-\frac{1}{2} k_{a b} m \epsilon^{\mu \nu \alpha} A_{\mu}^{a} \partial_{\nu} A_{\alpha}^{b}
$$

which describes an abelian chiral theory for $\varphi^{a}$ coupled as a pure gauge field to an abelian Chern-Simons theory for $A_{\mu}^{a}$, containing torsion.

\section{B. Nonlinear deformation}

It is now of obvious interest to consider the compatibility of torsion with the Yang-Mills and Freedman-Townsend types of covariant deformations of this linear abelian gauge theory for the potential $A_{\mu}^{a}$. Because the abelian gauge symmetry $\delta_{\zeta} A_{\mu}^{a}$ is independent of torsion, the closure equations up to 1st order for determining the linear deformation terms in the gauge symmetry continue to have solutions of the Yang-Mills and Freedman-Townsend types

$$
\stackrel{(1)}{\delta}_{\zeta} A_{\mu}^{a}=\kappa_{\mathrm{YM}} a_{b c}^{a} A_{\mu}^{b} \zeta^{c}+\kappa_{\mathrm{FT}} b_{b c}^{a} * \tilde{F}_{\mu}^{b} \zeta^{c}
$$


where the constant coefficient tensors $a_{a b c}=k_{a d} a_{b c}^{d}$ and $b_{a b c}=k_{a d} b_{b c}^{d}$ satisfy the algebraic relations (3.15) and (3.16), and $\kappa_{\mathrm{YM}}, \kappa_{\mathrm{FT}}$ are independent coupling constants. Note these deformation terms are constructed covariantly from the potential $A_{\mu}^{a}$ and the torsion field strength $* \tilde{F}_{a}^{\mu}$. A straightforward analysis of the determining equations for the quadratic deformation terms in the field equation yields

$$
\stackrel{(3)}{L}=\frac{1}{2} \kappa_{\mathrm{YM}} a_{b c}^{a} \epsilon_{\mu}{ }^{\nu \alpha} * \tilde{F}_{a}^{\mu} A_{\nu}^{b} A_{\alpha}^{c}+\frac{1}{2} \kappa_{\mathrm{FT}} b_{c}^{a b} \epsilon_{\mu \nu}{ }^{\alpha} * \tilde{F}_{a}^{\mu} * \tilde{F}_{b}^{\nu} A_{\alpha}^{c}+\frac{1}{6} m \epsilon^{\mu \nu \alpha} \kappa_{\mathrm{YM}} a_{a b c} A_{\mu}^{a} A_{\nu}^{b} A_{\alpha}^{c}
$$

together with the algebraic relations (3.17) and (3.19) in addition to

$$
p_{d[a}\left(a_{b] c}^{d}-m \kappa b_{b] c}^{d}\right)=0
$$

where $\kappa=\kappa_{\mathrm{FT}} / \kappa_{\mathrm{YM}}$. Notice that, up to this order in the deformation analysis, only the algebraic relation (4.37) involves torsion. The algebraic structure (3.15), (3.16), (3.17), (3.19), (4.37) comprises all integrability conditions at 1st and 2nd orders. This establishes the following main result.

Theorem 4.2: The algebraic condition (4.37) is the only obstruction to combining YangMills and Freedman-Townsend types of deformation of the linear abelian theory with torsion (4.23)-(4.28), including a Chern-Simons mass.

As in the case with no torsion, the other algebraic relations require $a_{b c}^{a}$ to be the structure constants of a semisimple Lie algebra on $\mathbb{V}$ whose Cartan-Killing metric is $k_{a b}=-a_{a c}^{d} a_{b d}^{c}$. In the massless case, the torsion obstruction states $p_{d[a} a_{b] c}^{d}=0$. Contraction with $a_{e}^{b c}$ leads to $p_{a e}=\frac{1}{2} a_{b a e} p^{b}$ by the Jacobi identity, where $p^{b}=p_{a d} a^{a d b}$. However, contraction with $a_{e}^{a b}$ gives $0=a^{a b}{ }_{e} a_{b c}^{d} p_{a d}=\frac{1}{2} p^{b} a_{b c e}$, which implies the vector $p^{b}$ lies in the center of the Lie algebra $\left(\mathbb{V}, a_{b c}^{a}\right)_{\mathrm{YM}}$. Hence $p^{b}$ vanishes since the Lie algebra is semisimple, and so $p_{a e}=0$. This algebraic analysis therefore gives a strong rigidity result.

Corollary 4.3: At lowest order, the only deformation of covariant form (4.35)-(4.36) that is compatible with torsion in the linear abelian theory in the massless case (without Chern-Simons term) consists of the pure Freedman-Townsend type.

In contrast, the torsion obstruction in the massive case has a quite different nature. Recall, the algebraic relations (3.16) and (3.17) without torsion hold if $b_{b c}{ }^{a}$ is identified with $a_{b c}^{a}$ as the structure constants of a semisimple Lie algebra on $\mathbb{V}$ and if $\kappa$ equals $1 / m$. 
This algebraic structure also trivially satisfies the obstruction (4.37), and thus the combined Yang-Mills and Freedman-Townsend type of covariant deformation at lowest order is compatible with torsion in the linear abelian theory, when the Chern-Simons mass is nonzero. A construction of this deformation to all orders amounts to turning on the YangMills Chern-Simons interaction in the torsion Freedman-Townsend gauge theory. Alternatively, the construction can be carried out more directly by adding torsion to the combined Freedman-Townsend Yang-Mills gauge theory with Chern-Simons term presented earlier, as will be now shown.

As with the pure Freedman-Townsend gauge theory, the introduction of torsion is accomplished by generalizing the symmetric linear map $Y_{\mathrm{A}_{\mu b}}^{a \nu}$ to $\tilde{Y}_{\mathrm{A}_{\mu b}}^{a \nu}$, which enters into constructing the nonlinear torsion field strength appearing in the gauge symmetry, field equation, and Lagrangian. This generalization is a nonlinear analog of replacing the metrics $g^{\mu \nu}$ and $k_{a b}$ in the Lagrangian of the linear abelian gauge theory (3.1) by the symmetric inner product $\tilde{g}_{a b}^{\mu \nu}$ to arrive at the Lagrangian for the torsion theory (4.23).

Thus, consider a general semisimple Lie algebra $\left(\mathbb{V}, c^{a}{ }_{b c}\right)=\mathfrak{g}$ and put $\kappa_{\mathrm{FT}}=m^{-1} \kappa_{\mathrm{YM}}$. Now introduce the torsion field strength

$$
\tilde{K}_{\mu}^{a}=\tilde{Y}_{\mathrm{A} \mu b}^{-1 a \nu} * F_{\mathrm{A} \nu}^{b}
$$

where $* F_{\mathrm{A}}$ is the Yang-Mills dual field strength (3.22) and $\tilde{Y}_{\mathrm{A}}$ is the linear map (4.20) with inverse $\tilde{Y}_{\mathrm{A}}^{-1}$. The complete Lagrangian with torsion is given by combining the Chern-Simons Lagrangian and the Freedman-Townsend torsion Lagrangian with the field strength replaced by $\tilde{K}_{\mu}^{a}$,

$$
\begin{aligned}
L_{\text {tors. }} & =\frac{1}{2} \tilde{Y}_{\mathrm{A}_{a b}^{\mu \nu}}^{\mu \nu} \tilde{K}_{\mu}^{a} \tilde{K}_{\nu}^{b}+\frac{1}{2} m \kappa_{\mathrm{YM}} a_{a b c} A_{\alpha}^{a}\left(\partial_{\mu} A_{\nu}^{b}+\frac{1}{3} a_{c d}^{b} A_{\mu}^{c} A_{\nu}^{d}\right) \\
& =\frac{1}{2} \tilde{g}_{a b}^{\mu \nu} \tilde{K}_{\nu}^{b}\left(m A_{\mu}^{a}+\tilde{K}_{\mu}^{a}\right)-\frac{1}{2} \kappa_{\mathrm{YM}} m^{-1} a_{a b c} \epsilon^{\mu \nu \alpha}\left(\left(m A_{\mu}^{a}+\tilde{K}_{\mu}^{a}\right) \tilde{K}_{\nu}^{b}+m^{2} \frac{1}{6} A_{\mu}^{a} A_{\nu}^{b}\right) A_{\alpha}^{c} .
\end{aligned}
$$

Similarly, the gauge symmetry on $A_{\mu}^{a}$ is obtained by replacing $\tilde{K}_{\mu}^{a}$ for the field strength in $\delta_{\zeta} A_{\mu}^{a}$ for Freedman-Townsend torsion theory. Under this gauge symmetry

$$
\delta_{\zeta} A_{\mu}^{a}=\partial_{\mu} \zeta^{a}+\kappa_{\mathrm{YM}} a_{b c}^{a}\left(A_{\mu}^{b}+m^{-1} \tilde{K}_{\mu}^{b}\right) \zeta^{c},
$$

the Lagrangian (4.39) is invariant to within a divergence. In the field equation obtained 
from this Lagrangian,

$$
E_{a}^{\mu}=g^{\mu \nu} k_{a b}\left(m * F_{\nu}^{b}+\epsilon_{\nu}^{\alpha \beta}\left(\partial_{\alpha} \tilde{K}_{\beta}^{b}+\kappa_{\mathrm{YM}} \frac{1}{2} \tilde{K}_{\alpha}^{c}\left(m^{-1} \tilde{K}_{\beta}^{d}+2 A_{\beta}^{d}\right)\right)\right.
$$

substitution of $* F_{\mathrm{A}}=\tilde{Y}_{\mathrm{A}} \tilde{K}$ leads to the elegant simplification

$$
\epsilon_{\nu}^{\alpha \beta}\left(\tilde{\partial}_{\alpha} \tilde{K}_{\beta}^{b}+\kappa_{\mathrm{YM}} \frac{1}{2} m^{-1} \tilde{K}_{\alpha}^{c} \tilde{K}_{\beta}^{d}\right)=-m \tilde{K}_{\mu}^{a}
$$

where $\tilde{\partial}_{\mu}$ is the derivative operator (4.30) given by the flat connection $m p^{a}{ }_{b} v_{\mu}$ for Lie-algebra valued differential forms as introduced in the linear abelian theory. Likewise, the field strength divergence equation coming from the Yang-Mills Bianchi identity $0=D_{\mathrm{A}}^{\mu} * F_{\mathrm{A}_{\mu}}^{a}$ simplifies to

$$
\tilde{\partial}^{\mu} \tilde{K}_{\mu}^{a}+Q_{b c}^{a} v^{\mu \nu} \tilde{K}_{\mu}^{b} \tilde{K}_{\nu}^{c}=0
$$

on solutions of the field equation, where $Q^{a}{ }_{b c}=\frac{3}{2} k^{a e} p_{d[e} c_{b c]}^{d}$ is the torsion tensor on $\mathbb{V}$.

Some field-theoretic properties of this novel torsion generalization of Yang-Mills ChernSimons theory are worth noting. The gauge group generated by the gauge symmetry on solutions is an infinite-dimensional representation of $\mathfrak{g}$,

$$
\left[\delta_{\zeta_{1}}, \delta_{\zeta_{2}}\right]=\delta_{\zeta_{3}}, \quad \zeta_{3}{ }^{a}=c^{a}{ }_{b c} \zeta_{1}^{b} \zeta_{2}^{c}
$$

Remarkably, the field strength for solutions is gauge invariant under this gauge group,

$$
\delta_{\zeta} \tilde{K}_{\mu}^{a}=0
$$

A natural subgroup of the gauge group consists of rigid symmetries

$$
\delta_{\left.\zeta\right|_{\text {rigid }}} A_{\mu}^{a}=\left.\kappa_{\mathrm{YM}} c^{a}{ }_{b c}\left(A_{\mu}^{a}+m^{-1} \tilde{K}_{\mu}^{a}\right) \zeta^{c}\right|_{\text {rigid }}
$$

where $\left.\zeta^{c}\right|_{\text {rigid }}=$ const. (note the dimension of this subgroup is equal to the internal number of fields, i.e. the dimension of $\mathfrak{g})$. Application of Noether's theorem to these symmetries yields a set of conserved currents

$$
J_{\mu}^{a}=c^{a}{ }_{b c} \epsilon_{\mu}{ }^{\nu \alpha} \tilde{K}_{\nu}^{b} \tilde{K}_{\alpha}^{c}+m \tilde{g}_{\mu b}^{a \nu} \tilde{K}_{\nu}^{b}
$$

which are gauge invariant $\delta_{\zeta} J_{\mu}^{a}=0$ and divergence free $\partial^{\mu} J_{\mu}^{a}=0$ on solutions $A_{\mu}^{a}$. Given a two dimensional hypersurface $\Sigma$ in spacetime $M$, the flux of these currents defines internal 
charges carried by the field $A_{\mu}^{a}, Q^{a}=\int_{\Sigma} J_{\mu}^{a} d \Sigma^{\mu}$ where $\epsilon_{\mu \nu \alpha} d \Sigma^{\mu}$ is the volume element pulled back to $\Sigma$. For solutions $A_{\mu}^{a}$, these charges are evaluated by a line integral around the boundary at infinity on $\Sigma$ by Stokes' theorem, $Q^{a}=\int_{\partial \Sigma} K_{\mu}^{a} d S^{\mu}$ where $d S^{\mu}$ is the line element on $\partial \Sigma$. When there is no current flow across $\partial \Sigma$, the charges are conserved and gauge invariant.

Under the action of diffeomorphisms on spacetime, the Lagrangian gives rise to a stressenergy tensor

$$
T_{\mu \nu}=k_{a b}\left(\tilde{K}_{\mu}^{a} \tilde{K}_{\nu}^{b}-\frac{1}{2} g_{\mu \nu} g^{\alpha \beta} \tilde{K}_{\alpha}^{a} \tilde{K}_{\beta}^{b}\right)-p_{a b} \frac{1}{2} \epsilon_{\mu}^{\alpha \beta} v_{\nu} \tilde{K}_{\alpha}^{a} \tilde{K}_{\beta}^{b}
$$

which is conserved $\partial^{\mu} T_{\mu \nu}=0$ and gauge-invariant $\delta_{\zeta} T_{\mu \nu}=0$ for solutions of the field equation. Notice, this stress-energy tensor is non-symmetric, due to the torsion term. As a result, contraction of $T_{\mu \nu}$ with a Killing vector $\xi^{\nu}$ of the spacetime metric $\mathcal{L}_{\xi} g^{\mu \nu}=-2 \partial^{(\mu} \xi^{\nu)}=0$ yields a conserved current $\partial^{\mu}\left(T_{\mu \nu} \xi^{\nu}\right)=0$ if $\xi^{\nu}$ is either a translation, or a rotation/boost in a hyperplane orthogonal to the vector $v^{\nu}$, i.e. $\mathcal{L}_{v} \xi^{\mu}=v_{\nu} \partial^{[\mu} \xi^{\nu]}=0$. If a two dimensional hypersurface $\Sigma$ in spacetime $M$ is considered as before, these conserved currents then define gauge-invariant fluxes of energy-momentum and angular momentum carried by the field $A_{\mu}^{a}$ on $\Sigma, Q_{\xi}=\int_{\Sigma} T_{\mu \nu} \xi^{\nu} d \Sigma^{\mu}$.

\section{Geometrical structure}

The nonlinear structure of this theory has several striking aspects that merge important geometrical features of pure Freedman-Townsend theory with torsion and combined YangMills Freedman-Townsend Chern-Simons theory without torsion. Here it will be useful to return to an index-free notation, $\mathbf{A}=A_{\mu}^{a} d x^{\mu} \mathbf{e}_{a}$ and $\mathbf{K}=K_{\mu}^{a} d x^{\mu} \mathbf{e}_{a}$. Write $\mathbf{v}=v_{\mu} d x^{\mu}$ as a covariantly constant 1-form on $M$ and $p(\cdot)=p^{a}{ }_{b} \mathbf{e}_{a} \mathbf{e}^{b}$ as a constant linear map on $\mathbb{V}$. Regard the torsion tensor $Q^{a}{ }_{b c}$ on $\mathbb{V}$ as a bilinear skew-symmetric map $Q(\cdot)$ from $\mathbb{V} \times \mathbb{V}$ into $\mathbb{V}$. To proceed, firstly, the field equation has the geometrical content that the Lie-algebra valued 1-form $\mathbf{A}+m^{-1} \tilde{\mathbf{K}}=\mathbf{A}_{\text {flat }}$ is a flat connection

$$
\mathbf{R}_{A_{\text {flat }}}=d \mathbf{A}_{\text {flat }}+\frac{1}{2}\left[\mathbf{A}_{\text {flat }}, \mathbf{A}_{\text {flat }}\right]=0
$$

This connection therefore has a zero-curvature form given by

$$
\mathbf{A}_{\text {flat }}=U^{-1} d U
$$


where $U$ is a chiral field, namely a function from spacetime into the simply-connected Lie group of matrices whose Lie algebra is $\mathfrak{g}$. Secondly, the field strength part $m^{-1} \tilde{\mathbf{K}}$ of this flat connection satisfies the curl and divergence equations

$$
\begin{aligned}
& m^{-1} \tilde{d} \tilde{\mathbf{K}}+\frac{1}{2} m^{-2}[\tilde{\mathbf{K}}, \tilde{\mathbf{K}}]=-\tilde{\mathbf{K}}, \\
& m^{-1} \tilde{d} * \tilde{\mathbf{K}}+m^{-2} \mathbf{v} \wedge Q(\tilde{\mathbf{K}} \wedge \tilde{\mathbf{K}}),
\end{aligned}
$$

involving the exterior derivative $\tilde{d}=d-m \mathbf{v} p(\cdot)$ on Lie-algebra valued differential forms on $M$. Note this derivative obeys $\tilde{d}^{2}=0$ and reduces to $\tilde{d}=d$ when acting on Liealgebra valued functions on $M$. The respective field strength equations (4.51) and (4.52) geometrically comprise a nonabelian Chern-Simons theory with a Proca mass term for the 1-form potential $m^{-1} \tilde{\mathbf{K}}=\mathbf{A}_{\text {mass. }}$ in a nonabelian Lorentz-torsion gauge

$$
* \tilde{\mathbf{F}}_{A_{\text {mass. }}}+m \mathbf{A}_{\text {mass. }}=0, \quad \widetilde{\operatorname{div}} \mathbf{A}_{\text {mass. }}+* \mathbf{v} \cdot Q\left(\mathbf{A}_{\text {mass. }} \wedge \mathbf{A}_{\text {mass. }}\right)=0
$$

with $\tilde{\mathbf{F}}_{A_{\text {mass. }}}=\tilde{d} \mathbf{A}_{\text {mass. }}+\frac{1}{2}\left[\mathbf{A}_{\text {mass. }}, \mathbf{A}_{\text {mass. }}\right]$. Solutions $\mathbf{A}_{\text {mass. }}$ describe a set of coupled spin-one vector fields of mass $m$ whose propagation on spacetime involves a "twist" effect due to the nature of $\tilde{d}$ discussed in the linear abelian theory. Moreover, if the same nonabelian Lorentz-torsion gauge condition is imposed on $\mathbf{A}_{\text {flat }}$,

$$
\widetilde{\operatorname{div}} \mathbf{A}_{\text {flat }}+* \mathbf{v} \cdot Q\left(\mathbf{A}_{\text {flat }} \wedge \mathbf{A}_{\text {flat }}\right)=0
$$

then $U$ is found to satisfy a "twisted" massless chiral field equation

$$
0=\widetilde{\operatorname{div}}\left(U^{-1} \widetilde{\operatorname{grad}} U\right) \text { or equivalently } \widetilde{\square} U=\widetilde{\operatorname{grad}} U \cdot\left(U^{-1} \widetilde{\operatorname{grad}} U\right) .
$$

In particular, solutions $U$ describe a set of coupled massless spin-zero matrix fields exhibiting "twist" in their propagation on spacetime. Note the gauge condition (4.54) can be achieved through a suitable finite gauge transformation on $\mathbf{A}_{\text {flat }}$, since the gauge symmetry acts as

$$
\delta_{\zeta} \mathbf{A}_{\text {flat }}=d \boldsymbol{\zeta}+\left[\mathbf{A}_{\text {flat }}, \boldsymbol{\zeta}\right], \quad \delta_{\zeta} \mathbf{A}_{\text {mass. }}=0
$$

on solutions of the field equation. Therefore, the 1-form potentials $\mathbf{A}_{\text {flat }}$ and $\mathbf{A}_{\text {mass. }}$ give a geometrical decomposition of $\mathbf{A}=\mathbf{A}_{\text {flat }}-\mathbf{A}_{\text {mass. }}$ into massive and massless parts.

Without the torsion-Lorentz gauge on $\mathbf{A}_{\text {flat }}$, the chiral field $U$ exhibits a pure gauge coupling to the massive Yang-Mills field $\mathbf{A}_{\text {mass. }}$. The precise nature of this coupling is best 
seen through the dual formulation of the torsion Lagrangian $L_{\text {tors. }}$ obtained by elimination of $\tilde{\mathbf{K}}$ in 1st order form, which yields

$$
-\frac{1}{m} L_{\text {dual }}^{\text {tors. }}=m \tilde{g}\left(U^{-1} d U-\mathbf{A}, U^{-1} d U-\mathbf{A}\right)+* \operatorname{tr}\left(-\mathbf{A} \wedge\left(\mathbf{D}_{U}(-\mathbf{A})+\frac{1}{3}[-\mathbf{A},-\mathbf{A}]\right)\right)
$$

in terms of $-\mathbf{A}=\mathbf{A}_{\text {mass. }}-U^{-1} d U$, where $\mathbf{D}_{U}=d+\left(U^{-1} d U\right)=U^{-1} d(U \cdot)$ is a zero-curvature derivative operator, $\left(\mathbf{D}_{U}\right)^{2}=0$.

Lastly, A, $\mathbf{A}_{\text {flat }}, \mathbf{A}_{\text {mass. }}$ are also linked geometrically by the action of the gauge group

$$
\mathbf{D}_{A_{\text {flat }}} \delta_{\zeta} \mathbf{A}=\left[\mathbf{R}_{A_{\text {flat }}}, \boldsymbol{\zeta}\right]=\tilde{\mathbf{Y}}_{A}\left(\delta_{\zeta} \mathbf{A}_{\text {mass. }}\right)
$$

where $\tilde{\mathbf{Y}}_{A}=\mathbf{1}-*(\mathbf{v} \wedge p(\cdot))-*[\mathbf{A}, \cdot]$, as holds from the deformation theorem 2.8 in Sec. [II]

\section{GRAVITY-LIKE YANG-MILLS CHERN-SIMONS THEORY WITH TOR- SION}

Gravity-like interactions of a 1-form potential $A_{\mu}^{a}$ arise in a natural fashion from consideration of non-covariant deformations involving stress-energy currents for linearized Yang-Mills gauge theory. Recall, in the linear abelian theory (3.1)-(3.7), such currents are produced from the stress-energy tensor

$$
\stackrel{(2)}{T}_{\mu \nu}=k_{a b}\left(* F_{\mu}^{a} * F_{\nu}^{b}-\frac{1}{2} g_{\mu \nu} g^{\alpha \beta} * F_{\alpha}^{a} * F_{\beta}^{b}\right)
$$

when it is contracted with any Killing vector $\xi^{\mu}$ of the flat spacetime metric $g_{\mu \nu}$. The current $\stackrel{(2)}{J}_{\mu}(\xi)=\stackrel{(2)}{T}_{\mu \nu} \xi^{\nu}$ is conserved $\partial^{\mu} \stackrel{(2)}{J}_{\mu}(\xi)=0$ and gauge invariant $\stackrel{(0)(2)}{\delta}_{\zeta}^{J}{ }_{\mu}(\xi)=0$ under the abelian gauge symmetry (3.2) on solutions $A_{\mu}^{a}$ of the linear field equation (3.6). As a starting point to write down a pure gravity-like deformation of the linear abelian theory, consider a set of commuting Killing vectors

$$
0=\mathcal{L}_{\xi_{a}} g^{\mu \nu}=-2 \partial^{(\mu} \xi_{a}^{\nu)}, \quad 0=\mathcal{L}_{\xi_{a}} \xi_{b}^{\mu}=2 \xi_{[b}^{\nu} \partial_{\nu} \xi_{a]}^{\mu}=\left[\xi_{b}, \xi_{a}\right]^{\mu}
$$

equal in number to the dimension of the internal vector space $\mathbb{V}$, i.e. so there is the same number of fields $A_{\mu}^{a}$ as Killing vectors $\xi_{a}^{\mu}$. In terms of such Killing vectors, the deformation to lowest order is given by

$$
\stackrel{(1)}{\delta}_{\zeta}^{a} A_{\mu}=\kappa_{\mathrm{G}} 2 \zeta^{b} \xi_{b}^{\nu} F_{\nu \mu}^{a}
$$


and

$$
\stackrel{(3)}{L}=\kappa_{\mathrm{G}} g^{\mu \nu} \stackrel{(2)}{J}_{\mu}\left(\xi_{a}\right) A_{\nu}^{a}
$$

with coupling constant $\kappa_{\mathrm{G}}$. Due to the explicit dependence on $\xi_{a}^{\mu}$, the deformation terms break Lorentz covariance.

An equivalent, more geometrical form for the deformed gauge symmetry is obtained if the gauge parameter is redefined by $\zeta^{\prime a}=\zeta^{a}+\kappa_{\mathrm{G}} A_{\mu}^{a} \xi_{b}^{\mu} \zeta^{b}$, yielding

$$
\stackrel{(1)}{\delta}_{\zeta} A_{\mu}^{a}=\kappa_{\mathrm{G}} \mathcal{L}_{\zeta} A_{\mu}^{a}, \quad \zeta^{\nu}=\zeta^{a} \xi_{a}^{\nu}
$$

through the Lie derivative identity $\mathcal{L}_{\zeta} f_{\mu}=\zeta^{\nu} \partial_{[\nu} f_{\mu]}+\partial_{\mu}\left(\zeta^{\nu} f_{\nu}\right)$ holding for any 1-form $f$ and vector field $\zeta$ on $M$. The gravity-like nature of the deformation is seen from two observations. First, this gauge symmetry (5.5) expresses an infinitesimal diffeomorphism invariance on $A_{\mu}^{a}$ with respect to the vector field $\zeta^{\nu}$ as the gauge parameter. Second, the Lagrangian (5.4) is given by a stress-energy self-coupling

$$
\stackrel{(3)}{L}=\frac{1}{2} T^{\mu \nu} \stackrel{(1)}{g}_{\mathrm{A}_{\mu \nu}}, \quad \stackrel{(1)}{g}_{\mathrm{A}}^{\mu \nu}=g_{\mu \nu}+2 A_{(\mu}^{a} g_{\nu) \alpha} \xi_{a}^{\alpha}+\cdots
$$

involving a deformation of the spacetime metric $g_{\mu \nu}$ to $g_{\mathrm{A}}$. These deformation terms (5.5) and (5.6) are analogous to the lowest-order nonlinear terms in Einstein gravity theory for a vielbein. Notice, however, the field equation for $A_{\mu}^{a}$ is not of a gravity-like form as it dynamically describes a set of spin-one electromagnetic field equations on $F_{\mu \nu}^{a}$, with current sources proportional to $\stackrel{(2)}{J}_{\mu}\left(\xi_{a}\right)$. In particular, there is no auxiliary gauge freedom on $A_{\mu}^{a}$, in contrast with the nature of the spin-two graviton field equation. (Indeed, as is well known, in three spacetime dimensions the graviton field equation has no dynamical degrees of freedom, once the linearized local Lorentz rotation freedom on the vielbein is taken into account.)

This type of deformation (5.5)-(15.6) can be generalized to use non-commuting Killing vectors by including a Yang-Mills interaction whose Lie algebra structure constants are tied to the Killing vector commutators, which is explained in Ref. [27] where the deformation is completed to all orders. A further generalization to include a Freedman-Townsend interaction can also be considered, which will be pursued elsewhere. Here the objective instead will be derive a torsion generalization of the pure gravity-like deformation of the linear abelian theory, extended to include a Chern-Simons term. 
For incorporating torsion, as well as a Chern-Simons term, it is essential to keep track of how the spacetime metric $g_{\mu \nu}$ and volume form $\epsilon_{\mu \nu \alpha}$ appear in the linear theory and the deformation terms. To proceed, write the abelian field strength as

$$
* F_{a}^{\mu}=k_{a b} \epsilon^{\mu \nu \alpha} \partial_{\nu} A_{\alpha}^{b}
$$

so the Lagrangian of the linear theory with an abelian Chern-Simons term takes the form

$$
\stackrel{(2)}{L}+\stackrel{(2)}{L}_{\mathrm{CS}}=\frac{1}{2} k^{a b} g_{\mu \nu} * F_{a}^{\mu} * F_{b}^{\nu}+\frac{1}{2} m A_{\mu}^{a} * F_{a}^{\mu}
$$

where the only dependence on the spacetime metric occurs through $g_{\mu \nu}$ in $\stackrel{(2)}{L}$. Torsion will now be added in the simplest manner by replacing the joint metric $k^{a b} g_{\mu \nu}$ (on vector fields with values in the dual space of $\mathbb{V}$ on $M$ ) with

$$
\tilde{g}_{\mu \nu}^{a b}=g_{\mu \nu} k^{a b}+v_{\mu \nu} p^{a b}, \quad v_{\mu \nu}=\epsilon_{\mu \nu \alpha} v^{\alpha}
$$

where, similarly to the torsion introduced previously, $v^{\mu}$ is a constant vector on $M$ and $p^{a b}$ is a constant skew tensor on $\mathbb{V}$. This yields the torsion Lagrangian

$$
\stackrel{(2)}{L}_{\text {tors. }}=\frac{1}{2} \tilde{g}_{\mu \nu}^{a b} * F_{a}^{\mu} * F_{b}^{\nu}+\frac{1}{2} m A_{\mu}^{a} * F_{a}^{\mu}
$$

with the following field-theoretic content. On solutions of the linear field equation

$$
\stackrel{(1)}{E}^{\mu a}+\stackrel{(1)}{E}^{\mathrm{CS} \mu a}=\epsilon^{\mu \nu \alpha} \tilde{g}_{\alpha \beta}^{a b} \partial_{\nu} * F_{b}^{\beta}+m k^{a b} * F_{b}^{\mu}=0,
$$

the curl of $\tilde{g}_{\alpha \beta}^{a b} * F_{b}^{\beta}+A_{\alpha}^{a}$ vanishes, hence

$$
* F_{b}^{\nu}=\left(\partial_{\mu} \varphi^{a}-m A_{\mu}^{a}\right)
$$

with $\varphi^{a}$ representing a chiral field, as before. Then $\stackrel{(2)}{L}_{\text {tors. }}$ has an equivalent dual formulation consisting of an abelian chiral Lagrangian theory for $\varphi^{a}$ minimally coupled to an abelian Chern-Simons theory for $A_{\mu}^{a}$, in which the torsion appears through the inverse metric $\tilde{g}_{a b}^{-1 \mu \nu}$ in contrast to the construction of the torsion Lagrangian (4.23).

\section{A. Torsion deformation}

Compatibility of the gravity-like deformation at lowest order with torsion in the linear abelian theory (5.7) -(5.10) is easily ascertained from an analysis of the determining equations 
for the deformation terms. The closure equation at 0th order for the linear deformation terms in the gauge symmetry remains satisfied, because the addition of a torsion term and a Chern-Simons term in the free Lagrangian does not affect the abelian gauge symmetry on $A_{\mu}^{a}$. At 1 st order, the determining equation for the quadratic terms in the field equation is found to yield

$$
\stackrel{(3)}{L}=\kappa_{\mathrm{G}} * F_{a}^{\mu} * F_{b}^{\nu}\left(A_{\mu}^{c} \tilde{g}_{\alpha \nu}^{a b}-\frac{1}{2} A_{\alpha}^{c} \tilde{g}_{\mu \nu}^{a b}\right) \xi_{c}^{\alpha}
$$

under the algebraic condition

$$
2 g_{\alpha \beta} v^{\beta[\nu} \partial^{\mu]} \xi_{a}^{\alpha}=\epsilon_{\alpha}{ }^{\mu \nu} v_{\beta} \partial^{[\alpha} \xi_{a}^{\beta]}=0 .
$$

This is the only obstruction to the deformation arising to this order. Remarkably, no other obstructions occur to all higher orders.

This obstruction has a simple content. Recall, for a rotation or boost Killing vector $\xi^{\nu}$ in a three-dimensional spacetime, $\partial^{[\mu} \xi^{\nu]}$ is a constant antisymmetric tensor whose dual vector $\epsilon_{\mu \nu}^{\alpha} \partial^{\mu} \xi^{\nu}$ gives the rotation/boost axis in spacetime; for a translation Killing vector, $\partial^{[\mu} \xi^{\nu]}$ vanishes.

Theorem 5.1: For any flat spacetime metric $g_{\mu \nu}$ in three dimensions, there are two classes of commuting Killing vectors $\xi_{a}^{\nu}$ : (i) translations, comprising a two or three dimensional planar isometry subgroup of the metric; (ii) a rotation or boost plus an orthogonal translation, comprising a two dimensional axisymmetric isometry subgroup of the metric. The algebraic obstruction (5.14) is satisfied for class (i) by any vector $v^{\mu}$, and by a vector $v^{\mu}$ parallel to the rotation/boost axis for class (ii). Stated geometrically, $\mathcal{L}_{v} \xi_{a}^{\mu}=0$ for both classes.

Construction of the gravity-like deformation based on this structure now proceeds as follows. Geometrically, the gauge symmetry is given by

$$
\delta_{\zeta} A_{\mu}^{a}=\partial_{\mu} \zeta^{a}+\mathcal{L}_{\zeta^{c} \xi_{c}} A_{\mu}^{a}
$$

where the coupling constant has been absorbed into the Killing vectors $\xi_{a}^{\mu}$. The Lagrangian is constructed using a deformed metric $\tilde{g}_{\mathrm{A}_{\mu \nu}}^{a b}=k^{a b} g_{\mathrm{A}_{\mu \nu}}+p^{a b} v_{\mathrm{A}_{\mu \nu}}$ given by

$$
\begin{aligned}
& g_{\mathrm{A}_{\mu \nu}}=g_{\mu \nu}+2 A_{(\mu}^{c} g_{\nu) \alpha} \xi_{c}^{\alpha}+A_{\mu}^{c} A_{\nu}^{d} g_{\alpha \beta} \xi_{c}^{\alpha} \xi_{d}^{\beta} \\
& v_{\mathrm{A}_{\mu \nu}}=v_{\mu \nu}+2 A_{[\mu}^{c} v_{\nu] \alpha} \xi_{c}^{\alpha}+A_{\mu}^{c} A_{\nu}^{d} v_{\alpha \beta} \xi_{c}^{\alpha} \xi_{d}^{\beta}
\end{aligned}
$$


Under the gauge symmetry (5.15), this metric has the crucial property

$$
\delta_{\zeta} \tilde{g}_{\mathrm{A}}^{a b}=\mathcal{L}_{\zeta^{c} \xi_{c}} \tilde{g}_{\mathrm{A}_{\mu \nu}}^{a b}
$$

by means of the algebraic relation (5.14). Then a gauge-invariant Lagrangian to within a divergence is obtained by

$$
L=\frac{1}{2}\left(\operatorname{det} g_{\mathrm{A}}\right)^{-1 / 2} \tilde{g}_{\mathrm{A}}^{a b} * F_{a}^{\mu} * F_{b}^{\nu}+\frac{1}{2} m A_{\mu}^{a} * F_{a}^{\mu}
$$

where det $g_{\mathrm{A}}$ denotes the standard determinant of the metric (5.16) in spacetime coordinates. Gauge invariance is verified through Lie derivative formulas combined with the properties

$$
\begin{aligned}
& \delta_{\zeta} \ln \left(\operatorname{det} g_{\mathrm{A}}\right)=g_{\mathrm{A}}^{-1 \mu \nu} \delta_{\zeta} g_{\mathrm{A}_{\mu \nu}}=g_{\mathrm{A}}^{-1 \mu \nu} g_{\mathrm{A}_{\mu \nu}}=\zeta^{c} \xi_{c}^{\alpha} \partial_{\alpha} \operatorname{det} g_{\mathrm{A}}+2 \operatorname{det} g_{\mathrm{A}} \partial_{\alpha}\left(\zeta^{c} \xi_{c}^{\alpha}\right), \\
& \delta_{\zeta} * F_{a}^{\mu}=k_{a b} \epsilon^{\mu \nu \alpha} \partial_{\nu} \delta_{\zeta} A_{\alpha}^{b}=\mathcal{L}_{\zeta^{c} \xi_{c}} * F_{a}^{\mu}+* F_{a}^{\mu} \partial_{\alpha}\left(\zeta^{c} \xi_{c}^{\alpha}\right) .
\end{aligned}
$$

In particular, it is found that

$$
\delta_{\zeta} L=\partial_{\alpha}\left(\zeta^{c} \xi_{c}^{\alpha} L\right)
$$

which clearly exhibits the gravity-like nature of the gauge invariance.

Note the determinant factor in the Lagrangian can be written in a more familiar form if a deformed abelian field strength is introduced

$$
*_{\mathrm{A}} F_{a}^{\mu}=k_{a b} \epsilon_{\mathrm{A}}^{\mu \nu \alpha} F_{\nu \alpha}^{b}=\left(\operatorname{det} g_{\mathrm{A}}\right)^{-1 / 2} * F_{a}^{\mu},
$$

through a deformation of the volume tensor $\epsilon_{\mathrm{A}}^{\mu \nu \alpha}=\left(\operatorname{det} g_{\mathrm{A}}\right)^{-1 / 2} \epsilon^{\mu \nu \alpha}$ corresponding to the deformed metric (5.16). This yields

$$
L=\frac{1}{2}\left(\operatorname{det} g_{\mathrm{A}}\right)^{1 / 2}\left(\left(\tilde{g}_{\mathrm{A}_{\mu \nu}}^{a b} *_{\mathrm{A}} F_{a}^{\mu}+m A_{\nu}^{b}\right) *_{\mathrm{A}} F_{b}^{\nu}\right.
$$

An interesting geometrical feature of this theory comes from writing the gauge symmetry as a covariant derivative

$$
\delta_{\zeta} A_{\mu}^{a}=\partial_{\mu} \zeta^{\prime a}+2 F_{\nu \mu}^{a} \xi_{b}^{\nu} k_{\mathrm{A}}{ }_{c}{ }^{\zeta^{\prime c}}, \quad \zeta^{\prime a}=k_{\mathrm{A}}{ }_{b}{ } \zeta^{b}
$$

using a redefinition of the gauge parameter, where $k_{\mathrm{A}}{ }_{b}{ }_{b}=\delta_{b}{ }^{a}+\xi_{b}^{\alpha} A_{\alpha}^{a}$. The covariant curl of this gauge symmetry yields the curvature of the connection 1-form $\Gamma_{\mu b}^{a}=2 F_{\nu \mu}^{a} \xi_{b}^{\nu} k_{\mathrm{A}}{ }_{c}^{b} \zeta^{\prime c}$,

$$
D_{[\mu} \delta_{\zeta} A_{\nu]}^{a}=R_{\Gamma \mu \nu b}^{a} \zeta^{b}
$$


In agreement with the geometrical deformation theorem 2.8, this curvature is related to the deformed abelian field strength through the action of the gauge symmetry

$$
R_{\Gamma \mu \nu b}^{{ }^{a} \zeta^{b}}=\epsilon_{\mathrm{A} \mu \nu \rho} Y_{\mathrm{A}}^{-1}{ }_{\alpha}^{\rho} k^{a b} \delta_{\zeta}\left(Y_{\mathrm{A}_{\beta}}^{\alpha} *_{\mathrm{A}} F_{b}^{\beta}\right)
$$

where $Y_{\mathrm{A}_{\beta}}{ }^{\alpha}=\delta_{\beta}{ }^{\alpha}+\xi_{c}^{\alpha} A_{\beta}^{c}$. Furthermore, the linear map $Y_{\mathrm{A}}$ here has a vielbein-like relation to the deformed metric $\tilde{g}_{\mathrm{A}_{\mu \nu}}^{a b}=\tilde{g}_{\alpha \beta}^{a b} Y_{\mathrm{A}_{\mu}}^{\alpha} Y_{\mathrm{A}_{\nu}}{ }^{\beta}$.

\section{CONCLUDING REMARKS}

The novel nonlinear generalization of Yang-Mills Chern-Simons gauge theory with torsion in three spacetime dimensions constructed in Sec. [V] has an extension to four dimensions when the 1 -form potentials $A_{\mu}^{a}$ are coupled to 2 -form potentials $B_{\mu \nu}^{a}$, similarly to the case without torsion derived in Ref. [16]. Indeed the construction extends more generally to a tower of coupled $p$-form potentials for $1 \leq p \leq d-2$ in $d \geq 3$ dimensions, where torsion is naturally motivated starting from a dual chiral field formulation of the Freedman-Townsend field strength of the $d-2$-form potential $B_{\mu_{1} \cdots \mu_{d-2}}^{a}$. A Yang-Mills self-interaction of the 1-form potentials $A_{\mu}^{a}$, combined with a Yang-Mills Higgs interaction between the potentials $B_{\mu_{1} \cdots \mu_{d-2}}^{a}$ and $A_{\mu}^{a}$, is expected to be compatible with torsion if a $d$-dimensional Chern-Simons type term is included in the theory, analogously to the case in $d=3$ dimensions. It would be of interest to explore whether similar considerations apply to the gravity-like interaction of 1 -form potentials in $d \geq 3$ dimensions.

In $d=3$ dimensions, there is a special type of torsion that yields an integrable chiral field equation due to Ward [31]. This torsion field theory arises by a dimensional reduction of the chiral formulation of self-dual Yang-Mills equations in $2+2$ dimensions and exhibits soliton-like solutions and other characteristic features of integrability such as a hierarchy of symmetries and conservation laws. Geometrically, the integrability involves parallelism [28], i.e. flattening of the curvature on the Lie group for the target space of the chiral field, as produced by adding torsion to the Cartan-Killing connection. For the torsion to precisely cancel the Riemannian part in the curvature, it must be proportional to the Lie algebra structure tensor of the Lie group. If a dual formulation is considered for such a chiral field parallelism theory, it leads to the condition $Q_{b c}^{a}=\lambda c_{b c}^{a}=\frac{3}{2} k^{a e} p_{d\left[e^{c}{ }_{b c]}{ }_{b c}\right.}$, where $Q_{b c}^{a}$ is the torsion, $p_{a b}$ is the torsion potential, $c^{a}{ }_{b c}$ denotes the Lie algebra structure constants and 
$k_{a b}=-c^{d}{ }_{a c} c^{c}{ }_{b d}$ is the Cartan-Killing metric, pulled back to the Lie algebra of the Lie group. However, for a semisimple Lie group, contraction of this condition with $c_{d a}^{c}$ followed by $k^{b d}$ yields $\lambda=0$. Thus, Ward's integrable chiral field equation has no dual formulation as a gauge field theory of 1-form potentials.

It then becomes worthwhile to ask if a self-dual gauge theory exists that contains a Freedman-Townsend interaction of $d-2$-form potentials in $d \geq 3$ dimensions, as this could lead to an interesting dual chiral formulation or perhaps to integrability properties. Of course, self-duality in $d$ dimensions requires pairing $p$-form potentials with $d-p-2$-form potentials. The simplest case would be 1-form potentials coupled to scalar (0-form) fields by an extended Freedman-Townsend type interaction in $d=3$ dimensions, in analogy with three dimensional self-dual Yang-Mills Higgs theory. A natural suggestion is thus to investigate a deformation of self-dual Yang-Mills Higgs theory by including a Freedman-Townsend interaction. 
[1] C.N. Yang and R.L. Mills, Phys. Rev. 96 (1954) 191-.

[2] S. Deser, Gen. Rel. Grav. 1 (1970) 9-18.

[3] R.M. Wald, Ph. Rev. D 33 (1986) 3613-3625.

[4] V.I. Ogievestky and I.V. Polubarinov, Ann. Phys. (NewYork) 35 (1965) 167-208.

[5] J. Fang and C. Frondsdal, J. Math. Phys. 20 (1979) 2264-2271.

[6] S.C. Anco, Contemp. Math. 132 (1992) 27-50.

[7] M. Henneaux, Contemp. Math. 219 (1998) 93-109; G. Barnich and M. Henneaux, Phys. Lett. B311 (1993) 123-.

[8] J. Stasheff, Contemp. Math. 219 (1998) 195-210; J. Stasheff and J. Heubschmann, Forum Mathematicum 14 (2002) 847-868.

[9] M. Henneaux, Nucl. Phys. B (Proc. Suppl.) A18 (1990) 47-; G. Barnich, M. Henneaux, R. Tatar, Int. J. Mod. Phys. D3 (1994) 139-; G. Barnich, F. Brandt, M. Henneaux, Comm. Math. Phys. 174 (1995) 57-; ibid., Comm. Math. Phys. 174 (1995) 93-.

[10] J. Stasheff, R. Fulp, T. Lada, in Proceedings of the 22nd Winter School (Srni, 2002) "Geometry and Physics", Rend. Circ. Mat. Palermo (2) Suppl. No. 71 (2003) 115-126.

[11] M. Henneaux and B. Knaepen, Phys. Rev. D 56 (1997) 6076-6080; ibid., Int. J. Mod. Phys. A15 (2000) 3535-3548.

[12] G. Barnich, F. Brandt, M. Henneaux, Nucl. Phys. B455 (1995) 357-; G. Barnich, F. Brandt, M. Henneaux, Phys. Rep. 338 (2000) 439-569.

[13] N. Boulanger, T. Damour, L. Gualtieri, M. Henneaux, Nucl. Phys. B597 (2001) 127-171.

[14] S.C. Anco, Ann. Phys. (N.Y.) 270 (1998) 52-125.

[15] S.C. Anco, Class. Quantum Grav. 19 (2002) 6445-6467.

[16] S.C. Anco, J. Math. Phys. 44 (2003) 1006-1043.

[17] J. Isenberg, D. Bao, and P. Yasskin, in Mathematical aspects of superspace, eds. H.-J. Seifert, C.J.S. Clarke, and A. Rosenblum (D. Reidel, Dorecht Holland 1983).

[18] C. Cutler and R.M. Wald, Class. Quantum Grav. 4 (1987) 1267-1278; R.M. Wald, Class. Quantum Grav. 4 (1987) 1279-1316.

[19] N. Boulanger and L. Gualtieri, Class. Quantum Grav. 18 (2001) 1485-1502.

[20] S.C. Anco, Phys. Rev. D 67 (2003) 124007. 
[21] S.C. Anco, J. Math. Phys. 36 (1995) 6553-6565.

[22] S.C. Anco, J. Math. Phys. 38 (1997) 3399-3413.

[23] D.Z. Freedman and P.K. Townsend, Nucl. Phys. B 177 (1981) 282-296.

[24] F. Brandt and N. Dragon, in Proceedings of the 31st International Symposium (Ahrenshoop, Buckow) on Theory of Elementary Particles, eds. H. Dorn, D. Lüst, G. Weigt (Wiley-VCH, Wienheim 1998).

[25] F. Brandt, in Proceedings of NATO Advanced Research Workshop (2001) "Noncommutative structures in mathematics and physics".

[26] S.C. Anco, Lett. Math. Phys. 62 (2002) 245-258.

[27] F. Brandt, Phys. Rev. D 64 (2001) 065025.

[28] E. Braaten, T.L. Curtright, C.K. Zachos, Nuc. Phys. B 260 (1985) 630-688.

[29] C.N. Yang, Phys. Rev. Lett. 38 (1977) 1377-1379.

[30] K. Pohlmeyer, Comm. Math. Phys. 72 (1980) 37-47.

[31] R.S. Ward, Nonlinearity 1 (1988) 671-679.

[32] V.E. Zakharov and A.V. Mikhailov, Sov. Phys. JEPT 47 (1978) 1017-1027; M. Forger and P. Zizzi, Nuc. Phys. B 287 (1987) 131-143; E. Witten, Comm. Math. Phys. 92 (1984) 455-472.

[33] S.C. Anco and J. Isenberg, Comm. Partial Diff. Eqs. 25 (200) 1669-1702.

[34] J. Shatah and M. Struwe, Geometric Wave Equations (AMS, Providence 2000).

[35] P.J. Olver, Applications of Lie Groups to Differential Equations (Springer, New York 1986).

[36] I.M. Anderson, Contemp. Math. 132 (1992) 51-74.

[37] S.C. Anco and G. Bluman, Euro. Jour. Appl. Math. 13, 545-566 (2002); ibid., Euro. Jour. Appl. Math. 13, 567-585 (2002).

[38] J. Stasheff, R. Fulp, T. Lada, Sh-Lie algebras Induced by Gauge Transformations, Comm. Math. Phys. 231 (2002) 25-43; J. Stasheff, G.Barnich, R. Fulp and T.Lada, The sh Lie structure of Poisson brackets in field theory, Comm. Math. Phys. 191 (1998) 585-601.

[39] G. Bluman and S.C. Anco, Symmetry and Integration Methods for Differential Equations (Springer, New York 2002).

[40] P. Van Nieuwenhuizen, Phys. Rep. 68 (1980) 189-.

[41] See, for example: A. Sagle and R. Walde, Introduction to Lie Groups and Lie Algebras (Academic Press 1973).

[42] S. Helgason, Differential Geometry, Lie Groups, and Symmetric Spaces (AMS, Providence 
2001). 